\title{
Vortices and waves in light dark matter
}

\author{
Lam Hui, ${ }^{a, *}$ Austin Joyce, ${ }^{a, b, \dagger}$ Michael J. Landry, ${ }^{a, \ddagger}$ and Xinyu Li ${ }^{a, c, d, \S}$ \\ ${ }^{a}$ Center for Theoretical Physics, Department of Physics, \\ Columbia University, New York, NY 10027, USA \\ ${ }^{b}$ Delta-Institute for Theoretical Physics, \\ University of Amsterdam, Amsterdam, 1098 XH, The Netherlands \\ ${ }^{c}$ Canadian Institute for Theoretical Astrophysics, \\ University of Toronto, 60 St George St, Toronto, ON M5R 2M8, Canada \\ ${ }^{d}$ Perimeter Institute, \\ 31 Caroline Street North, Waterloo, Ontario, Canada, N2L 2Y5, Canada
}

\begin{abstract}
In a galactic halo like the Milky Way, bosonic dark matter particles lighter than about $30 \mathrm{eV}$ have a de Broglie wavelength larger than the average inter-particle separation and are therefore well described as a set of classical waves. This applies to, for instance, the QCD axion as well as to lighter axion-like particles such as fuzzy dark matter. We show that the interference of waves inside a halo inevitably leads to vortices, locations where chance destructive interference takes the density to zero. The phase of the wavefunction has non-trivial winding around these points. This can be interpreted as a non-zero velocity circulation, so that vortices are sites where the fluid velocity has a non-vanishing curl. Using analytic arguments and numerical simulations, we study the properties of vortices and show they have a number of universal features: (1) In three spatial dimensions, the generic defects take the form of vortex rings. (2) On average there is about one vortex ring per de Broglie volume and (3) generically only single winding $( \pm 1)$ vortices are found in a realistic halo. (4) The density near a vortex scales as $r^{2}$ while the velocity goes as $1 / r$, where $r$ is the distance to vortex. (5) A vortex segment moves at a velocity inversely proportional to its curvature scale so that smaller vortex rings move faster, allowing momentary motion exceeding escape velocity. We discuss observational/experimental signatures from vortices and, more broadly, wave interference. In the ultra-light regime, gravitational lensing by interference substructures leads to flux anomalies of $5-10 \%$ in strongly lensed systems. For QCD axions, vortices lead to a diminished signal in some detection experiments but not in others. We advocate the measurement of correlation functions by axion detection experiments as a way to probe and capitalize on the expected interference substructures.
\end{abstract}

\footnotetext{
*1h399@columbia.edu

$\dagger$ a.p.joyce@uva.nl

${ }^{\ddagger}$ ml2999@columbia.edu

$\S$ xli@cita.utoronto.ca
} 


\section{Contents}

1 Introduction $\quad 3$

2 A general existence argument for defects $r$

2.1 A simple example: domain walls in $1+1$ dimensions 8

2.2 Vortices in higher dimensions 9

3 Simple analytic solutions and their properties $r$

3.1 Domain walls 12

3.2 Vortices 13

3.2.1 Static vortex lines 13

3.2.2 Moving vortex solutions 16

3.2.3 Vortex rings $\quad 16$

3.3 Configurations of vortices 18

4 The random phase model and the number density of vortices 19

4.1 The random phase model 19

4.2 The number density of vortices 23

5 Vortex configurations in simulated haloes with gravity 26

5.1 Numerical algorithm 27

5.2 Numerical solutions including gravity 28

6 Observational and experimental implications 32

6.1 Observational implications 34

6.1.1 Lensing magnification 34

6.1.2 Lensing deflection 38

6.2 Experimental implications 40

7 Conclusions $\quad 44$

A Solution-generating technique for the Schrödinger equation 48

B Gravitational lensing by a vortex 49

$\begin{array}{ll}\text { C Axion-photon interaction } & 50\end{array}$

$\begin{array}{ll}\text { D Axion-fermion interaction } & 51\end{array}$

E Real scalar correlations from the random phase model 52

$\begin{array}{lc}\text { References } & 53\end{array}$ 


\section{Introduction}

Though there is overwhelming evidence for the existence of dark matter from a variety of astronomical probes, the constituents of dark matter remain a mystery. Ideas range from a particle as light as $10^{-22} \mathrm{eV}$ to primordial black holes of tens of solar mass. One useful demarcation in this immense spectrum is the division between particle-like and wave-like dark matter. This boundary in phenomenology occurs at masses around $30 \mathrm{eV}$ in a galactic halo like the Milky Way. A dark matter particle with mass below this $30 \mathrm{eV}$ threshold has a de Broglie wavelength that exceeds the typical inter-particle separation. Such a particle is necessarily bosonic, because of the Pauli exclusion principle for fermions. ${ }^{1}$ In this case where the average particle number in a de Broglie volume is very large, the corresponding quantum state has very high occupation number and its evolution is well approximated by a classical field [2], much as a collection of many photons is well described by electric and magnetic fields satisfying Maxwell's equations. ${ }^{2}$ In this paper, we are interested in the classical wave-like behavior of dark matter in this regime.

There is a large literature on light dark matter of this sort, which is commonly modeled by a scalar or pseudo-scalar field [5-12]. A particularly appealing realization of the general framework is a pseudoNambu-Goldstone boson, a prime example of which is the QCD axion [13-22], which could span a large range of masses. Experimental efforts have focused in particular on masses around $10^{-6} \mathrm{eV}$, with some reaching down to much lower values (see reviews by [23-25]). More generally, axion-like-particles (ALPs) abound in string theory with a wide range of masses [26-29]. An intriguing possibility is an ALP with mass around $10^{-22}-10^{-20} \mathrm{eV}$, which under fairly simple assumptions has a relic abundance appropriate to be the dark matter. ${ }^{3}$ In this extreme low mass limit, the de Broglie wavelength is a significant fraction of the size of a galaxy, a possibility often referred to as fuzzy dark matter [30-37]. Beyond these concrete examples, the wave-like features we study will be present in any model where the dark matter is light enough for the number of quanta in a de Broglie volume to be large, e.g., [38-42].

For our purposes, the precise underlying model for dark matter is not crucial, nor is its mass as long as it is under $\sim 30 \mathrm{eV}$. What we do assume is that the dark matter is well-described by a classical scalar field whose only non-negligible interaction is gravitational. ${ }^{4}$ This is a reasonable approximation when the system is in a high-occupancy state, which in this context requires a sufficiently light dark matter mass.

\footnotetext{
${ }^{1}$ This is the origin of the Tremaine-Gunn bound [1] for fermionic dark matter.

${ }^{2}$ For example, a coherent state with a large average occupancy (i.e., many particles in a de Broglie volume) behaves classically in the sense that quantum fluctuations are negligible. A collection of heavy particles, on the other hand, is naturally described by a distribution function obeying the Boltzmann equation. In this case there is also an effective description in terms of classical fields after coarse-graining by taking the hydrodynamic limit. See e.g., [3, 4]. However, this situation is conceptually different and does not lead to wave-like phenomena on macroscopic scales.

${ }^{3}$ More precisely - assuming an initial mis-alignment angle of order unity - the relic abundance of a particle in this mass range is $\Omega_{\mathrm{ALP}} \sim 0.1\left(\mathrm{~m} / 10^{-22} \mathrm{eV}\right)^{1 / 2}\left(f / 10^{17} \mathrm{GeV}\right)^{2}$, where $m$ is the ALP mass and $f$ is the associated axion decay constant.

${ }^{4}$ For an axion, it can be shown self-interaction is subdominant compared to gravity or quantum pressure for a typical halo dark matter density. The relative importance of self-interaction, gravity and quantum pressure can be evaluated by comparing: $m^{2} \phi^{4} / f^{2},\left(m^{2} \phi^{2}\right)^{2} r^{2} / M_{\mathrm{Pl}}^{2}$ and $\phi^{2} / r^{2}$. It can be shown the first term is smaller than the other two for all $r$ for a fixed $\rho=m^{2} \phi^{2}$ as long as $1>10^{-11}\left(10^{-6} \mathrm{eV} / m\right)\left(10^{12} \mathrm{GeV} / f\right)^{2}\left(\rho / \mathrm{GeV} / \mathrm{cm}^{3}\right)^{1 / 2}$. The inequality also holds for fuzzy dark matter like values $m \sim 10^{-22} \mathrm{eV}$ and $f \sim 10^{17} \mathrm{GeV}$.
} 
Our starting point is to consider a real Klein-Gordon scalar $\phi$ minimally coupled to gravity, which is described by the following action

$$
S=\int \mathrm{d}^{4} x \sqrt{-g}\left(-\frac{1}{2}(\partial \phi)^{2}-\frac{m^{2}}{2} \phi^{2}\right) .
$$

We are interested in situations where the dark matter is non-relativistic. This applies to much of the structure in the universe, the primary exception being dark matter close to black holes (e.g., [43-48]). In the non-relativistic limit, it is convenient to parameterize the field as $^{5}$

$$
\phi=\frac{1}{\sqrt{2 m}}\left(\Psi e^{-i m t}+\Psi^{*} e^{i m t}\right)
$$

where $\Psi$ is a complex scalar. Assuming $\Psi$ is slowly varying compared to the frequency $m$, i.e., $|\ddot{\Psi}| \ll m|\dot{\Psi}|$, and dropping terms that oscillate rapidly, the Klein-Gordon equation implies that $\Psi$ satisfies a Schrödinger equation coupled to gravity ${ }^{6}$

$$
i \partial_{t} \Psi=-\frac{1}{2 m} \nabla^{2} \Psi+m \Phi \Psi
$$

where $\Phi$ is the gravitational potential, which is itself fixed by the Poisson equation sourced by the local mass density $\rho$

$$
\nabla^{2} \Phi=\frac{\rho}{2 M_{\mathrm{Pl}}^{2}} \quad \text { where } \quad \rho=m|\Psi|^{2}
$$

It is worth stressing that - despite the fact that the field $\Psi$ obeys a Schrödinger equation - this system of equations describes the classical evolution of the collective dynamics of the underlying dark matter particles. The wave-like nature of these classical field configurations allows for a variety of behaviors that are analogous to familiar quantum-mechanical phenomena, but it is important to keep in mind that the physics is purely classical. ${ }^{7}$ In this paper we will be interested in the dynamics of this SchrödingerPoisson system in gravitationally-bound objects like galaxies, so we neglect the background expansion of the universe, which is irrelevant on these scales. For details of the cosmology of light dark matter see, e.g., $[2,24,35,52-54]$.

In the early universe, $\Psi$ is roughly homogeneous with small perturbations, and linear perturbation theory suffices to describe the growth of structure. In the nonlinear regime, the fact that $\rho \propto|\Psi|^{2}$ leads to striking interference patterns - for instance, a galactic halo typically consists of a superposition of $\Psi$ waves leading to order unity fluctuations in the mass density $\rho$, as first demonstrated by Schive et al. [32]. This interference substructure has many interesting dynamical consequences, some of which were explored in [35]. In this paper, we are particularly interested in destructive interference - we study locations where chance interference takes the density all the way to zero. An illustration is shown in Figure 1, drawn from [54].

\footnotetext{
${ }^{5}$ The real and imaginary parts of the complex field, $\Psi$, parameterize the two phase space degrees of freedom of the (real) scalar, $\phi$. For a discussion of the systematics of the non-relativistic limit, see [49-51].

${ }^{6}$ Here and throughout this paper we normalize the wavefunction $\Psi$ so that $|\Psi|^{2}$ is a number density.

${ }^{7}$ In particular, though we have set $\hbar=1$, if we were to re-introduce these factors they would always appear in the combination $\hbar / m$, so that everything can be understood classically.
} 

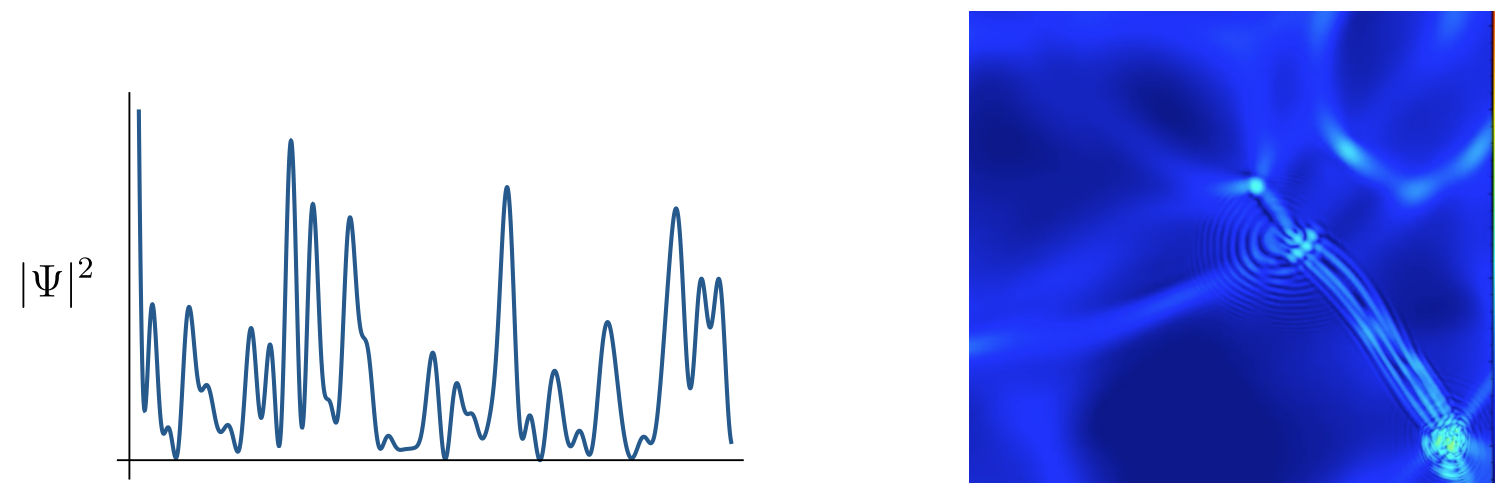

Figure 1: In the outer regions of a light dark matter halo, the dynamics is well captured by a random superposition of waves. Chance destructive interference can take the local dark matter density all the way to zero. In the left panel, as an example we show a $1 \mathrm{D}$ plot of $|\Psi|^{2}$, where $\Psi$ is constructed from a sum of plane waves with random phases. Note that there are many places where the square of the wavefunction vanishes. On the right, we show a more realistic scenario. This is a snapshot of the dark matter density in a cosmological simulation of ultra-light dark matter done in [54], showing interference fringes from wave-like superposition in dark matter filaments and haloes (darker shade means lower density). The locations where the dark matter density vanishes behave like vortices (see $\S 2$ ). At early times (e.g., in the initial conditions) there is no complete destructive interference because fluctuations are too small. However, these fluctuations are amplified by gravitational collapse after which destructive interference occurs. It turns out that many of the features of the vanishing loci can be understood without gravity. In this paper we explore the formation and properties of these vortex features.

What is so special about these sites of complete destructive interference? These loci behave essentially like topological defects around which the phase of $\Psi$ jumps or has a non-trivial winding, as will be explained in Section 2. ${ }^{8}$ They occur at a rate of about one per de Broglie volume in a typical halo, and they have universal density profiles, leading to interesting observational/experimental signatures. Our goal in this paper is to demonstrate and explore this phenomenology with a number of numerical and analytic computations. Gravity is important in the formation of these objects - in the early universe fluctuations in $\Psi$ are sufficiently small compared to the background value that nowhere is interference destructive enough to make $\rho$ vanish. The fluctuations of $\Psi$ are amplified by gravity until complete destructive interference is possible and, as we will see, generically occurs. Interestingly, once order one fluctuations in $\Psi$ are present, many of the features of these defects can be understood without gravity. We will present computations both with and without gravity to illustrate these points.

In many respects light dark mater resembles a superfluid, and many phenomena familiar from the study of superfluids have avatars in this setting. The topological defects of interest behave like vortices from this point of view. In order to understand the correspondence, let us recall the fluid (Madelung [55]) formulation of the Schrödinger equation. ${ }^{9}$ We start by re-expressing the wavefunction in terms of its amplitude and

\footnotetext{
${ }^{8}$ It is worth stressing that these are not the axion strings that form as a result of the spontaneous breaking of the PecceiQuinn $U(1)$ symmetry. Here, the relevant $U(1)$ symmetry is a more mundane one: that associated with particle number conservation. We discuss more how these defects should be thought of in the underlying relativistic theory in Section 7 .

${ }^{9}$ An elementary discussion can be found in the Feynman lectures [56].
} 
phase:

$$
\Psi=\sqrt{\frac{\rho}{m}} e^{i \theta}
$$

where $\rho$ and $\theta$ are interpreted as the fluid mass density and the velocity potential, respectively. In other words, we have

$$
\rho=m|\Psi|^{2}, \quad \vec{v}=\frac{1}{m} \vec{\nabla} \theta .
$$

In these variables, eq. (1.3) takes the form of the familiar equations of hydrodynamics, albeit with a somewhat peculiar source of stress. Inserting the parametrization (1.5) into the Schrödinger equation, the imaginary part gives a continuity equation for the density

$$
\partial_{t} \rho+\vec{\nabla} \cdot(\rho \vec{v})=0
$$

which expresses the conservation of mass in the fluid system. This equation can of course equally well be interpreted as the continuity equation for the probability current. The real part of the Schrödinger equation gives an independent Euler-like equation

$$
\partial_{t} \vec{v}+(\vec{v} \cdot \vec{\nabla}) \vec{v}=-\vec{\nabla} \Phi+\frac{1}{2 m^{2}} \vec{\nabla}\left(\frac{\nabla^{2} \sqrt{\rho}}{\sqrt{\rho}}\right) .
$$

The final term in (1.8) is often interpreted as a "quantum pressure," but this is somewhat misleading because it does not correspond to an isotropic stress (and in this context is not at all quantum). ${ }^{10}$ The change of variables between the Schrödinger formulation and the Madelung equations is highly nonlinear, which makes it possible to study solutions that are complicated in one set of variables in the other formulation, where the solutions can be substantially simpler. It is worth noting that the fluid variables obtained in this fashion are not the same as the ones that would appear in the hydrodynamic description obtained from phase space coarse graining (see footnote 2), though they will agree at long distances.

In the fluid formulation, the velocity field is a gradient flow, just as in a superfluid. At first sight, it therefore seems there is no room for vorticity. Moreover, Kelvin's theorem [57] - the co-moving conservation of circulation - follows from taking the curl of the Euler-like equation, which tells us that if the initial vorticity vanishes everywhere, it would continue to. ${ }^{11}$ How then can vortices develop? The obvious loophole arises from places where the density vanishes: there the phase $\theta$ and the velocity become ill-defined. As we will argue, the phase $\theta$ has a non-trivial winding around these loci, leading effectively to a non-zero velocity circulation $\oint \mathrm{d} \vec{\ell} \cdot \vec{v}$, i.e., vorticity $\vec{\nabla} \times \vec{v}$ is non-vanishing at such locations. ${ }^{12}$

\footnotetext{
${ }^{10}$ The quantum pressure term can be re-written in terms of a stress tensor as $\frac{1}{2 m^{2}} \partial_{i}\left(\frac{\nabla^{2} \sqrt{\rho}}{\sqrt{\rho}}\right)=\partial^{j} \sigma_{i j}$, where $\sigma_{i j}=$ $\frac{\rho}{4 m^{2}} \partial_{i} \partial_{j} \log \rho$, which typically has off-diagonal elements.

${ }^{11}$ More explicitly the curl of the Euler equation is $\partial_{t}(\vec{\nabla} \times \vec{v})^{i}+v^{k} \nabla_{k}(\vec{\nabla} \times \vec{v})^{i}+(\vec{\nabla} \cdot \vec{v})(\vec{\nabla} \times \vec{v})^{i}-S^{i j}(\vec{\nabla} \times \vec{v})^{j}=0$ where $S^{i j} \equiv \partial^{(i} v^{j)}$. So, if the initial curl is zero, it will remain zero.

${ }^{12}$ Another way to say this is that Kelvin's theorem fails for the Madelung fluid because of the points where the density vanishes. Neither the fluid velocity nor the so -called quantum pressure are well defined at these points. Vorticity (the velocity integrated along a closed loop) does not need to be conserved if the loop intersects a point where the fluid parameterization is ill-defined [58, 59].
} 
We can therefore interpret the loci where the wavefunction vanishes as vortices. Note that this requires the vanishing of both the real and imaginary parts of the wavefunction. Since the wavefunction is a function of $d=D-1$ spatial variables, the vanishing of either its real or imaginary part generically occurs along a co-dimension one surface. These two surfaces will generically intersect along a co-dimension two surface i.e., a string in $d=3$. These strings are vortices from the fluid perspective. They do not end, and thus generically take the form of vortex rings. In lower dimension - or in non-generic circumstances in three spatial dimensions - the total wavefunction can vanish along a lower co-dimension surface, leading to the appearance of a domain wall from the fluid perspective. What we will show is that the phase and density close to a defect (be it a wall or a vortex) behave in characteristic ways, and that defects occur with a predictable frequency in a galactic halo. ${ }^{13}$

Vortices are ubiquitous in physics. They appear, for example, as defects in the Abelian Higgs model [63], as flux tubes in confining gauge theories [64] and in both superfluids [65] and in Bose-Einstein condensates (BECs) [66]. It is worth emphasizing, however, that the vortices we consider in this paper differ somewhat from the vortices that arise in these situations. In particular, the fluid we consider behaves like a superfluid, but with self-interactions turned off. In our context, the density is not a gapped degree of freedom, so there does not appear to be a well-defined effective field theory of these defects along the lines of [64, 67-71]. Even within the context of wave-like dark matter vortices are well-studied [72-77]. However, most of the previous studies have focused on the regime of BEC dark matter where self-interactions dominate over the quantum pressure term (sometimes called the Thomas-Fermi regime). Precisely the opposite regime is of interest on galactic scales for weakly-coupled dark matter with a large de Broglie length, and it is this regime that we focus on in this paper. In particular we combine simple analytical estimates in the absence of gravity with numerical simulations to ensure that our conclusions are robust to the inclusion of gravity and to verify that the vortex configurations have the properties we expect. Some properties of vortex solutions of the Schrödinger equation were also studied numerically in [78].

We find that vortex-like defects in light dark matter have a number of universal properties which persist even in the presence of gravity. As we justify below, on general grounds we expect that vortices will have unit winding, and will have a characteristic density profile $\rho \sim r^{2}$, and velocity profile $v \sim 1 / r$, near the vortex location. All of these expectations are borne out by numerical simulations. We further expect based both on dimensional arguments and on simple analytic estimates that the number density of defects should be approximately one per de Broglie volume, in good agreement with numerical results. We will investigate the implications of vortices, and more broadly interference substructures, for astrophysical observations (relevant for the ultra-light regime) and for axion detection experiments (relevant for light, but not necessarily ultra-light, dark matter). One feature we will use is the fact that vortices can potentially move at high speed. Some of the most interesting signatures have to do with general stochastic fluctuations

\footnotetext{
${ }^{13}$ A possible point of confusion is that in the large $m$ limit, the Schrödinger-Poisson equations should match smoothly onto a particle description of the dark matter fluid [60], which certainly can support vorticity without defects. The resolution of the apparent paradox is that the classical fluid description arises after coarse-graining. In particular, the Wigner function gives rise to a fluid phase space distribution function after smoothing on some scale, and vorticity can arise in the effective fluid as a byproduct of this coarse-graining procedure from structure smaller than the smoothing scale [61, 62].
} 
associated with the interference substructures.

The organization of this paper is as follows. In $\S 2$, we present a general Taylor-expansion argument to describe how $\Psi$ should behave close to a defect, from which we infer some general properties of defects. In $\S 3$, we explore simple, exact solutions when gravity is ignored, for walls $\S 3.1$ and vortices $\S 3.2$. We show how defects can form and disappear. In $\S 4$, we introduce a simple statistical model for the fluctuations of the light dark matter field in a galactic halo and use this model to estimate the number density of defects. In $\S 5$, we explore examples of defects in numerical simulations, which incorporate the effect of gravity, and show that their general characteristics are consistent with those of the simple, exact solutions. Possible observational/experimental signatures are discussed in $\S 6$. We conclude in $\S 7$ with a discussion of angular momentum and the role of vortices, along with future prospects for observational/experimental signatures. Several technical results are relegated to the Appendices. As this manuscript was in preparation, a preprint by [79] appeared on the subject of gravitational lensing flux anomaly. It has some overlap with our discussion in $\S 6$.

Conventions: We employ the mostly-plus metric signature. We denote the spacetime dimension by $D$ and the spatial dimension by $d$, though we primarily work in four spacetime dimensions. Throughout the paper we have set Planck's constant, $\hbar$, and the speed of light, $c$, to one.

\section{$2 \quad$ A general existence argument for defects}

Defects appear in fluid variables precisely at points where the square of the wavefunction vanishes, so that the parameterization (1.6) is no longer well-defined. This corresponds to locations where both the real and imaginary parts of the wavefunction are zero. We would like to understand how the system behaves under generic circumstances in the vicinity of such a defect. The system of equations (1.3)-(1.4) is non-linear, so the general dynamics of defects is complicated. However, very close to to the defect, it is possible to characterize the behavior in a universal way. We therefore first focus on the near-defect regime, before turning to numerical simulations to verify that defects behave as expected from our analytical arguments.

\subsection{A simple example: domain walls in $1+1$ dimensions}

It is instructive to first consider the case of one spatial dimension. In this situation, the only possible defect has co-dimension one, corresponding to a wall. We wish to demonstrate that the behavior of the density and velocity in the neighborhood of the wall is universal.

Suppose there is a certain location, say $x=0$, where the density happens to vanish (at some particular time $t$ ), meaning both the real and imaginary parts of $\Psi$ vanish. In the neighborhood of $x=0$, we can Taylor expand the wavefunction as (for simplicity we restrict to static configurations)

$$
\Psi(x) \sim x \partial_{x} \Psi(0)+\cdots,
$$

under the assumption that $\partial_{x} \Psi(0)$ does not vanish. In order for $\Psi$ to be well-defined as a complex function, its phase must jump by $\pi$ (or an odd integer multiple, $(2 n+1) \pi, n \in \mathbb{Z}$ ) as $x$ crosses zero. That is, the 


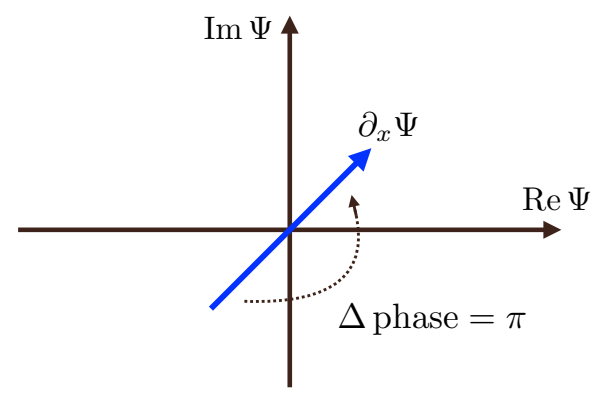

Figure 2: Behavior of the wavefunction in $1+1$ dimensions in the vicinity of a zero. As we move in the $x$ direction, the wavefunction varies along the (blue) arrow labeled by $\partial_{x} \Psi$. In order for the wavefunction to vanish at a point, but still have a finite first derivative the phase has to jump by $\pi$ across the origin, corresponding to the location of the domain wall.

sign of $\Psi$ changes as $x$ passes through zero. This indicates that there is a wall defect located at $x=0$. This situation is illustrated in Figure 2. It is possible to tune things such that there is no phase jump at the location of the wavefunction's zero, by arranging for odd derivatives of the field to vanish but this is highly non-generic.

Notice that - given this profile - as we move away from the location where the domain wall is located, the square of the wavefunction scales as

$$
\rho \sim|\Psi|^{2} \sim x^{2}
$$

so that the fluid density grows like $x^{2}$ in the vicinity of the defect.

\subsection{Vortices in higher dimensions}

In higher dimensions, the generic locus along which either the real or imaginary part of the wavefunction vanishes will have co-dimension one. The intersection of these two surfaces where the total wavefunction vanishes will generally be a co-dimension two surface. Therefore, in the particular case of three spatial dimensions, our expectation is that the generic defects will be take the form of lines. Further, it is difficult to arrange for the wavefunction to vanish along a line that extends all the way to infinity, so we should expect that the typical defects will be closed rings as opposed to infinite lines. As we will see in a moment, these lines/rings are vortices/vortex rings. This is consistent with our expectation that dark matter haloes will be of finite size and therefore will only be able to support vortex loops of finite extent.

The vorticity of a flow is given by the line integral of the velocity field around a closed loop

$$
\mathcal{C}=\oint_{\partial A} \mathrm{~d} \vec{\ell} \cdot \vec{v}=\int_{A} \mathrm{~d} \vec{A} \cdot \vec{\nabla} \times \vec{v}
$$

In the second equality we have used Stokes' theorem to write the circulation as an area integral. Since the fluid velocity is a gradient flow, this will always be zero unless the curl is singular somewhere, which happens exactly at the location of vortices. Additionally, if we do the line integral in (2.3) - possibly 
enclosing a vortex - the integral is related to the phase difference of the wavefunction transported around a closed circle, which must be an integer multiple of $2 \pi / m$ for the wavefunction to be single-valued [80]

$$
\mathcal{C}=\frac{1}{m} \oint_{\partial A} \mathrm{~d} \theta=\frac{2 \pi n}{m}, \quad \text { with } \quad n \in \mathbb{Z}
$$

We therefore see that the vorticity carried by the Schrödinger vortices must be quantized, as in a superfluid [65].

We can repeat the argument from Section 2.1 to see that in the generic case the lines along which the wavefunction vanishes indeed support vorticity. In the vicinity of a point (the origin, say) where the wavefunction vanishes, we Taylor expand the wavefunction as

$$
\Psi(\vec{x}) \simeq \vec{x} \cdot \vec{\nabla} \Psi(0)+\cdots
$$

where the higher-order corrections are unimportant at small distances. In the generic case, the wavefunction will vanish along a line (our eventual vortex), so that the derivative along the line direction will also vanish. Without loss of generality we take the tangent to the line to be pointing along the $z$ direction. It is then convenient to introduce complex coordinates in the transverse plane

$$
\mathfrak{z} \equiv x+i y, \quad \overline{\mathfrak{z}} \equiv x-i y,
$$

In these coordinates, the wavefunction in the near-vortex regime takes the form ${ }^{14}$

$$
\Psi(\mathfrak{z}, \overline{\mathfrak{z}}) \simeq \mathfrak{z} \bar{\partial} \Psi(0)+\overline{\mathfrak{z}} \partial \Psi(0)+\cdots \simeq a_{\mathfrak{z}}+b \overline{\mathfrak{z}}+\cdots
$$

The quantities $\partial \Psi(0)$ and $\bar{\partial} \Psi(0)$ are just (complex) constants, which we have written as $a$ and $b$ in the last equality. When $|a|>|b|$, the phase winds by $+2 \pi$ as we traverse a circle enclosing the origin, while in the opposite case $|a|<|b|$ the phase winds by $-2 \pi$ as we encircle the origin. ${ }^{15}$ So we see that both of these cases describe vortices, either of winding 1 or winding -1 .

In the vicinity of the vortex, we can work out the density profile in the plane. It is of the form

$$
\rho \sim|\Psi|^{2} \sim A x^{2}+B y^{2}+C x y
$$

where the parameters $A, B, C$ can be solved for in terms of $a, b$, but their precise form is not important. What we learn from this expression is that the density scales roughly as $\sim r_{\perp}^{2}$ (where $r_{\perp}$ is the radius in cylindrical coordinates - the distance from the vortex) but is not in general axially symmetric. Rather, lines of constant density are ellipses. Also, the fact that we have a definite winding means the fluid velocity $v \sim 1 / r_{\perp}$ around a vortex (see eqs. (2.3) and (2.4)). The momentum density $\rho v$ vanishes towards the vortex, scaling as $r_{\perp}$.

There are two degenerate cases: if $|a|=|b|$ then the configuration is not a vortex, but is instead a domain wall, where the phase jumps by $\pi$ as we cross the wall. A special case of this situation is when both $a$ and

\footnotetext{
${ }^{14}$ We define $\partial \equiv \frac{\partial}{\partial \mathfrak{z}}$ and $\bar{\partial} \equiv \frac{\partial}{\partial \bar{z}}$.

${ }^{15}$ This can be understood as a consequence of the argument principle, generalized to harmonic complex functions [81].
} 
$b$ vanish. In that case we have to keep the next order in the Taylor expansion and the defect is typically a vortex of higher winding, unless parameters are again tuned at this order. Our generic expectation is therefore that loci where the wavefunction vanishes in $3+1$ dimensions describe defects of winding \pm 1 .

We can also make some inferences about the dynamics of vortex lines using these arguments. In this case, we imagine Taylor expanding in the vicinity of a defect, but additionally allow the configuration to evolve in time so that we have

$$
\Psi(\vec{x}, t) \simeq \frac{i t}{2 m} \nabla^{2} \Psi(0)+\vec{x} \cdot \vec{\nabla} \Psi(0)+\cdots,
$$

where we have used the Schrödinger equation to relate the coefficient of the $t$ term to that of the $\mathcal{O}\left(x^{2}\right)$ terms. Here, 0 denotes the origin in both time and space where the wavefunction vanishes. Near the vortex, we can neglect the $\mathcal{O}\left(x^{2}\right)$ terms and again let us choose the tangent to the vortex to point in the $z$ direction. At some small $t$, we can infer the location of the vortex by setting (2.9) to zero, from which we read off the vortex velocity:

$$
\dot{\vec{x}} \cdot \vec{\nabla} \Psi(0)=-\frac{i}{2 m} \nabla^{2} \Psi(0) .
$$

By splitting the wavefunction into its real and imaginary parts as $\Psi=\Psi_{1}+i \Psi_{2}$ we can write this equation as a matrix equation for the instantaneous velocity in the plane perpendicular to the vortex:

$$
\left(\begin{array}{cc}
\partial_{x} \Psi_{1}(0) & \partial_{y} \Psi_{1}(0) \\
\partial_{x} \Psi_{2}(0) & \partial_{y} \Psi_{2}(0)
\end{array}\right)\left(\begin{array}{c}
\dot{x} \\
\dot{y}
\end{array}\right)=\frac{1}{2 m}\left(\begin{array}{c}
\nabla^{2} \Psi_{2}(0) \\
-\nabla^{2} \Psi_{1}(0)
\end{array}\right) .
$$

Inverting this matrix gives the local velocity, it scales roughly like the ratio between the coefficient of the $t$ term divided by the coefficients of the $\vec{x}$ terms. Dimensionally this scales as

$$
v_{\text {vortex }} \sim \frac{1}{m R}
$$

where $R$ is the characteristic scale over which the configuration varies. Notice that for curved vortices, this characteristic scale is of order the curvature, so we expect that the velocity is inversely proportional to the radius of curvature of the vortex, $R$. This is consistent with the behavior of superfluid vortices, where smaller vortex loops move faster. ${ }^{16}$

\section{Simple analytic solutions and their properties}

The arguments in Section 2 are based purely on the Taylor expansion of the wavefunction near its zeros, and are therefore quite robust. In particular, the fact that locations where $\Psi$ vanishes support defects is

\footnotetext{
${ }^{16}$ From the point of view of the Taylor expansion argument presented in this section, one might reasonably ask: why not expand around some other (non-vanishing) values of the wavefunction? What is so special about a vanishing wavefunction? The winding of the phase, the $1 / r_{\perp}$ velocity profile and the $r_{\perp}^{2}$ density profile are all special to the vortex where the wavefunction vanishes. On the other hand, the argument about velocity in eq. (2.11) works for any particular value of the wavefunction one chooses to focus on. This velocity is useful in the case of the vortex because it tells us how the center of this $r_{\perp}^{2}$ density profile moves. We will exploit this fact in discussing observational implications.
} 
essentially independent of the details of the theory and is not affected by the presence of gravity. However, to this point we have not actually solved the Schrödinger equation, so one might wonder whether these vortices are actually present in physical solutions, and whether vortices can form if they were initially absent. In this Section, we rectify this situation by verifying that there are solutions to the Schrödinger equation that have the expected properties. ${ }^{17}$

Deriving simple analytic solutions with vortices also allows us to get some intuition for how these objects behave. Since many of the properties of defects are quite generic, it is useful to first consider solutions in the absence of gravity. In particular, this simplified setting allows us to understand how defects can form and disappear, and gives insight into the density profiles in the vicinity of vortices, which will turn out to be universal. We expect that the coarse features of these solutions will continue to persist even in the presence of gravitational interactions, at least sufficiently close to the defect. We will also learn about the fluid flow pattern around a vortex ring, and how it relates to the ring's motion. We will study as well what happens when vortices encounter each other. In Section 5 we confirm many of the insights gained here by numerically simulating the formation and evolution of defects, in the presence of gravity.

\subsection{Domain walls}

To begin, we look for solutions that describe domain walls. These are the expected defects in $1+1$ dimensions, but should not occur generically in $3+1$ dimensions. We will see later on that indeed such defects do not form in the $3+1$ numerical simulations of a generic gravitational collapse.

First, let us focus on a static defect in one spatial dimension. With gravity turned off, the Schrödinger equation for time-independent wavefunctions takes the extremely simple form

$$
\partial_{x}^{2} \Psi=0
$$

so it is easy to write the most general solution to this equation as

$$
\Psi=x+b,
$$

where $b$ is a constant. Without loss of generality, we can set $b=0$ by choosing the origin appropriately. Thus, $x=0$ is where $\Psi$, and therefore $\rho$, vanishes. This is the location of the sought-after defect. The solution is so trivial in appearance that one might miss its structure. To make things more apparent, rewrite $\Psi$ as an amplitude and a phase:

$$
\Psi=|x| e^{i \theta}
$$

The phase, $\theta$, goes from $-\pi$ when $x<0$ to 0 for $x>0$. In other words, we learn a couple interesting things about this wall defect: the density goes like $x^{2}$ where $|x|$ is the distance to the defect, and the phase

\footnotetext{
${ }^{17} \mathrm{~A}$ small note about normalization: throughout this section we will be somewhat un-careful with the dimensions of the wavefunction, $\Psi$. The physical wavefunction should have dimensions such that $|\Psi|^{2}$ is a number density. However, since the solutions that we write down solve a linear equation, their overall normalization is arbitrary. We will therefore often set the dimension-ful scales in front of these solutions to 1, but they can be restored by multiplying by a suitable power of any length scale desired.
} 
$\theta$ experiences a jump. One might complain that this solution is not very realistic: the density diverges far away from the defect. Our claim is this: once gravitational interactions are included, the divergence at infinity is regulated, but the description of the density and phase close to the defect remains correct. This claim will be validated in the presence of gravity in Section 5. Gravitational effects should become important parametrically around the de Broglie wavelength $=2 \pi /(m v)$, where $v$ is the velocity dispersion in the gravitationally bound object.

\subsection{Vortices}

Next we turn to consider vortex configurations. In Section 2.2, we argued that these should be the generic defects in three spatial dimensions. In this Section we describe various simple analytic vortex configurations and study their properties in the absence of gravity. ${ }^{18}$ These solutions, and many more complicated ones, can be efficiently constructed utilizing the solution-generating technique of [82], which we review in Appendix A. Some of the solutions that we describe have appeared in the literature before (for example in [82]), but we wish to review their properties in any case in order to understand the basic features that vortex lines have.

Since vortices are localized at the intersection where both the real and imaginary parts of the wavefunction vanish, we can visualize the evolution of vortex configurations by considering how these vanishing loci evolve in time. In Figure 3 we plot the vanishing surfaces for both real and imaginary parts of the wavefunction for some simple examples.

\subsubsection{Static vortex lines}

Our goal is to find solutions to the free Schrödinger equation which describe a flow with vorticity. As mentioned before, this requires both the real and imaginary parts of the wavefunction to vanish along a line. We begin by considering the simplest possible vortex solution: an infinitely long straight vortex.

We first look for a time-independent axially-symmetric solution (independent of $t, z$ ). We therefore want to solve the Laplace equation in the $x y$ plane. It is convenient to work in the complex coordinates (2.6). In these variables, the Laplace equation takes the simple form

$$
\partial \bar{\partial} \Psi(\mathfrak{z}, \overline{\mathfrak{z}})=0 .
$$

As is well known, this equation is solved by an arbitrary (anti-)holomorphic function. First consider the holomorphic sector, $\Psi \propto \mathfrak{z}^{n}$. It is straightforward to see that this solution describes a vortex located at $x=y=0$ with winding $n$. Similarly, the anti-holomorphic solutions, $\Psi \propto \overline{\mathfrak{z}}^{n}$, describe anti-vortices of winding $-n$.

The simplest vortex, of winding 1 , is described by the wavefunction

$$
\Psi=\mathfrak{z}=x+i y,
$$

\footnotetext{
${ }^{18} \mathrm{As}$ in the domain wall case, we expect that these analytic solutions should be reliable close to the defect, and we will later estimate where gravitational corrections become important.
} 


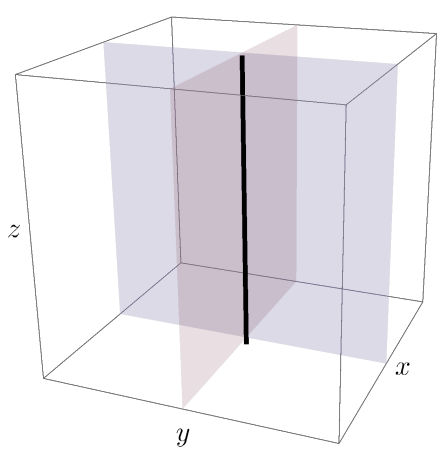

(a)

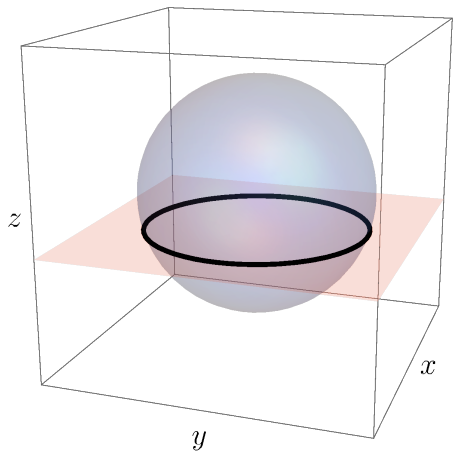

(b)

Figure 3: Loci where the real (blue) and imaginary (red) parts of the wavefunction $\Psi$ vanish for some examples. Vortices are localized on the co-dimension two strings where these surfaces intersect. a. Static vortex line configuration where $\operatorname{Re} \Psi$ vanishes on the plane $x=0$ (blue surface) and $\operatorname{Im} \Psi$ vanishes along the plane $y=0$ (red surface). The vortex line lies at their intersection along the $z$ axis. b. Dynamical nucleation of a vortex ring. The real part of the wavefunction $\operatorname{Re} \Psi$ vanishes along a sphere centered on the origin. Im $\Psi$ vanishes in a plane parallel to the $x y$ plane which moves in the $z$ direction with velocity $v_{z}$. Shown is the intersection of these two surfaces at some intermediate time in the ring's evolution.

The real part of the wavefunction vanishes along the $y z$ plane located at $x=0$ and the imaginary part vanishes along the $x z$ plane located at $y=0$. The intersection of these two planes is a line along the $z$ axis, where the total wavefunction vanishes and the vortex is located. In Figure 3 we visualize the intersection of the zero loci of the real and imaginary parts.

For purely holomorphic or anti-holomorphic vortices, the velocity flow is circular around the position of the vortex and it winds $+n$ or $-n$ times as we travel in a closed loop. However, we can consider an admixture of a vortex and anti-vortex solution

$$
\Psi \propto \mathfrak{z}+\alpha \overline{\mathfrak{z}}
$$

As was discussed in Section 2.2, depending on the size of $\alpha$, the solution will behave differently. For $\alpha<1$, this describes a winding $n=1$ solution, but the velocity flow will now be elliptical (equivalently, the change in the argument is no longer constant with angle as we travel around in a circle). Similarly, the lines of constant density will be ellipses in the $x y$ plane. Once we have $\alpha>1$, the solution describes an anti-vortex also with an elliptical flow. In the degenerate $\alpha=1$ case, the wavefunction happens to be purely real, and the solution now describes a domain wall in the $y z$ plane where the phase jumps by $\pi$ as we cross the wall. 


\section{Estimate where gravity becomes important}

For the simplest vortex solutions of winding 1, as we move away from the vortex core, the density grows as

$$
\rho(r)=m|\Psi|^{2}=\rho_{\text {local }}\left(\frac{r_{\perp}}{\lambda_{\mathrm{dB}}}\right)^{2},
$$

where $r_{\perp} \equiv \sqrt{x^{2}+y^{2}}$. The proportionality constant is fixed by assuming that the dark matter density in the vicinity of the vortex will grow until it reaches the local background density, denoted by $\rho_{\text {local }}$. The natural expectation is that this will occur around the de Broglie wavelength $\lambda_{\mathrm{dB}}=2 \pi /(m v)$.

This distance coincides with the characteristic length where we can no longer ignore gravitational effects. In the vicinity of vortices, gravity is unimportant, but due to the $r_{\perp}^{2}$ growth of the density, at some point it cannot be neglected. Said differently, we want to estimate when the $m \Phi \Psi$ term in (1.3) becomes important relative to the gradient (quantum pressure) term. If we assume that gradients in some region scale as the size of the region, $R$, the size of the quantum pressure term will decrease as we go to larger scales. When this term becomes of order the gravitational potential, we have $\Phi \sim(m R)^{-2}$. Inside a dark matter halo, the virial theorem tells us that the gravitational potential is nearly constant $\Phi \sim v^{2}$ so we are able to solve for $R$. We therefore find that gravitational effects become important at distance of order the de Broglie wavelength $R \sim \lambda_{\mathrm{dB}}$. This is perhaps expected because this is the characteristic length scale that governs all aspects of the dynamics. Note that once we include the effects of gravity, we expect the $r_{\perp}^{2}$ growth to stop and instead that the wavefunction will eventually vanish as $r_{\perp}$ exceeds the size of the halo, much like what happens for the Hydrogen atom.

\section{Hydrogen atom-like wavefunctions}

Another broad class of static solutions can be constructed similarly straightforwardly. These are the wavefunctions that solve the Laplace equation in spherical coordinates $(r, \theta, \varphi)$. In fact, any static solution can be decomposed in this basis of functions. In spherical coordinates, the static Schrödinger equation reads

$$
\nabla^{2} \Psi=\frac{1}{r^{2}} \partial_{r}\left(r^{2} \partial_{r} \Psi\right)+\frac{1}{r^{2}} \Delta_{S^{2}} \Psi=0
$$

where $\Delta_{S^{2}}$ is the Laplacian on the sphere. This equation is easily solved via separation of variables, leading to solutions of the form

$$
\Psi_{l, m}=r^{l} Y_{l}^{m}(\theta, \varphi)
$$

Here $Y_{l}^{m}$ are the standard spherical harmonics. ${ }^{19}$ These solutions are analogous to the wavefunctions of the hydrogen atom, only in this case there is no Coulombic potential. Similar to the hydrogen atom problem, we have imposed regularity of the wavefunction at $r=0$ as a boundary condition.

Not all of the wavefunctions (3.9) describe vortex configurations. Rather, only the ones with $m \neq 0$ do. As a special case the configurations with $m= \pm l$ reduce to the solutions considered above:

$$
r^{l} Y_{l}^{ \pm l} \sim(x \pm i y)^{l} .
$$

\footnotetext{
${ }^{19}$ We rely on context to differentiate $m$ as in $Y_{l}{ }^{m}$ versus $m$ as the mass of the dark matter particle.
} 
More generally, a solution with magnetic quantum number $m$ describes a vortex oriented in the $z$ direction with winding $m$. Aside from providing many examples of solutions with vortices, these solutions help illuminate the relation between angular momentum and the presence of vortices, which we discuss further in Section 7.

\subsubsection{Moving vortex solutions}

Once we have found a solution with a stationary vortex configuration, we can exploit the symmetries of the Schrödinger equation to obtain solutions with vortices moving at constant velocity. A general Schrödinger group transformation acts quite nontrivially on the wavefunction [83], but for our purposes, we only need the transformation of the wavefunction under galilean boosts: ${ }^{20}$

$$
\Psi(t, \vec{x}) \mapsto \exp \left(-i m \vec{x} \cdot \vec{v}-\frac{i m}{2} \vec{v}^{2} t\right) \Psi(t, \vec{x}+\vec{v} t),
$$

where $\vec{v}$ is the boost parameter. Using this we can transform a static configuration into one moving at velocity $\vec{v}$. For example, an infinitely long vortex line moving with velocity $-v$ in the $x$ direction is given by

$$
\Psi=e^{-i m x v-\frac{i m v^{2} t}{2}}(x+v t+i y) .
$$

It is also possible to generate boosted versions of more complicated vortex configurations. Note that these boosted configurations are somewhat peculiar in that the entire fluid is also boosted, so they do not represent solutions where only the vortex is moving through a fluid at rest.

\subsubsection{Vortex rings}

The infinitely long vortex lines discussed so far are the simplest configurations, and they capture some important aspects of the physics, but we do not expect that these precise configurations will arise in dark matter haloes, which are of finite extent. In more realistic situations, the generic defects will consist of closed vortex loops (consistent with the overall conservation of vorticity at infinity). We therefore would like to explore the properties of simplified vortex ring solutions. The simplest example is given by

$$
\Psi_{\text {ring }}(\vec{x}, t)=r_{\perp}^{2}-\frac{\ell^{2}}{4}+2 i\left(-a z+\frac{t}{m}\right)
$$

where $r_{\perp}=x^{2}+y^{2}$, and $a$ is a parameter with units of length. The real part of the wavefunction vanishes on a cylinder oriented in the $z$ direction with diameter $\ell$, while the imaginary part vanishes in the $x y$ plane with a $z$ coordinate that increases at a constant rate with time. This configuration therefore describes a circular ring of radius $\ell / 2$ moving at $v_{\text {ring }}=(a m)^{-1}$ in the $+z$ direction. Note that this velocity is the speed of the ring as measured relative to the rest frame of the fluid very far from the vortex.

We can gain some intuition for the properties of this solution by expanding $r_{\perp}=\ell / 2+\delta r_{\perp}$ so that the near-ring solution takes the form (at $t=0$ )

$$
\Psi_{\text {near-ring }}(\vec{x}, 0)=\ell \delta r_{\perp}-2 a i z .
$$

\footnotetext{
${ }^{20}$ The commutators of the Schrödinger algebra can be found, for example, in [84].
} 


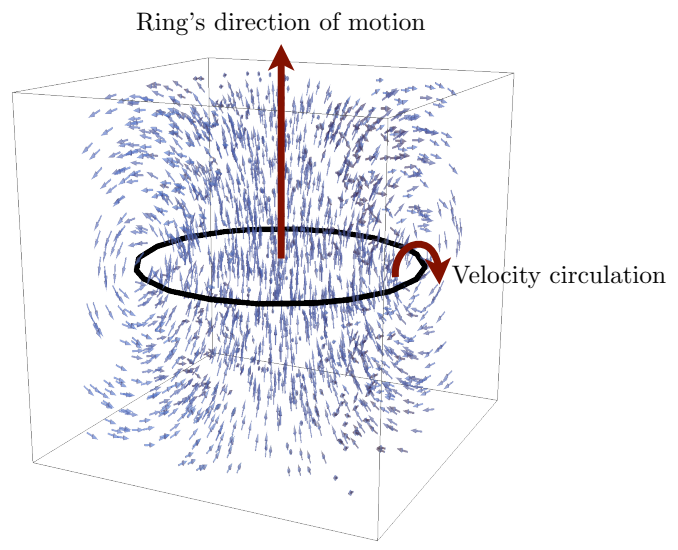

Figure 4: Cartoon of the velocity flow around a vortex ring moving in the $z$ direction. Note that the fluid velocity is such that it flows through the ring in the same direction that the ring is moving. The flow then circulates around the ring as shown.

In the $\left(\delta r_{\perp}, z\right)$ plane, we can write this in terms of the complex coordinate $u \equiv \delta r_{\perp}+i z$ as

$$
\Psi_{\text {near-ring }}(u, \bar{u})=(\ell / 2-a) u+(\ell / 2+a) \bar{u} .
$$

From this, we see that for a generic value of $a$, the fluid flow around this ring is non-circular, corresponding to a mix of a vortex and anti-vortex, though it will always have winding -1 . For $a=\ell / 2$, the near-ring geometry is precisely that of a pure vortex of winding -1 and in this case, there is a relation between the size of the ring and its velocity, $v_{\text {ring }} \sim(m \ell)^{-1}$, which is consistent with the behavior of superfluid vortex rings. We do not expect realistic vortex rings to be circular, but even in that case we generically expect the characteristic scale that governs the dynamics, $a$, to continue to be $a \sim \ell$, so that the relation between size and velocity persists.

In Figure 4 we show the velocity flow around a vortex ring with $a=\ell / 2$. Note in particular that the fluid velocity flows through the center of the ring in the same direction that the vortex moves. We also depict the velocity circulation around the ring. An interesting feature of the fluid flow is that the ring moves with a different velocity than the fluid around it. For example, at the very center of the ring, the fluid velocity is $v_{\text {fluid }}=2 v_{\text {ring }}$.

We can also investigate the density profile in the vicinity of a vortex ring. Very close to the ring, where the near-ring approximation (3.15) is reliable, the density grows as $r_{\perp}^{2}$ as expected, where $r_{\perp}$ is the distance from the vortex ring. Note however that very far from the ring, the density profile is anisotropic. In the plane of the ring, the density grows like $r_{\perp}^{4}$. However, in the transverse direction, the density only grows like $z^{2}$. These precise scalings depend on the fact that the ring in the solution lies in a plane, so we do not expect them to persist in more realistic scenarios, where the only truly robust signature will be $r_{\perp}^{2}$ growth of the density in the near-ring region. 


\section{Nucleation of a vortex ring}

An important feature of light dark matter vortices is that they can appear and disappear dynamically. We can understand this phenomenon in a simple analytic setting by considering the nucleation of a vortex ring. We take the real part of the wavefunction to vanish along a 2-sphere and have the imaginary part again vanish along a plane moving in the $z$ direction. The wavefunction in this case is given by:

$$
\Psi_{R}(\vec{x}, t)=r^{2}-\frac{\ell^{2}}{4}+i\left(-\ell z+\frac{3 t}{m}\right)
$$

where $r^{2}=x^{2}+y^{2}+z^{2}$. This describes the nucleation of a vortex ring at $x=y=0, z=-\ell / 2$ at $t=-\frac{m \ell^{2}}{6}$ which moves in the $z$ direction with velocity, $v=\frac{3}{m \ell}$. The radius of the ring at height $z$ is given by $R=\sqrt{\ell^{2} / 4-z^{2}}$. The vortex ring re-combines and annihilates at $t=\frac{m \ell^{2}}{6}$. This is visualized in Figure 3.

Note that the vortex ring moves at constant velocity in the $z$ direction, but the $v_{x}$ and $v_{y}$ components of points on the ring change as a function of its size, so that the magnitude of the ring's velocity increases as the size of the ring decreases. This behavior is consistent with our expectation from Taylor expansion (2.12), which suggests that smaller vortex rings move with faster linear velocity. In fact, as the ring shrinks to zero size, the velocity in the $x$ and $y$ directions becomes large, which may lead to interesting observational signatures, as we explore more in Section $6 .^{21}$

\subsection{Configurations of vortices}

It is also illuminating to consider slightly more complicated vortex configurations where vortex-anti vortex pairs spontaneously appear and disappear, as well as solutions where vortices intersect.

\section{Nucleation of vortex-anti-vortex pair}

If we choose the imaginary plane in eq. (3.16) to instead be oriented in the $y z$ direction, this will describe the intersection of a plane and a cylinder along two lines, which has the interpretation of the creation of a vortex-anti-vortex pair. We have a solution of the form ${ }^{22}$

$$
\Psi_{\mathrm{v} \overline{\mathrm{v}}}(\vec{x}, t)=x^{2}+y^{2}-\frac{\ell^{2}}{4}+2 i\left(-\frac{\ell x}{2}+\frac{t}{m}\right)
$$

For $t<-m \ell^{2} / 4$, this solution has no vortices. At $t=-m \ell^{2} / 4$ a vortex-anti-vortex pair nucleate at $x=-\ell / 2, y=0$, they move around the ring $x^{2}+y^{2}=\ell^{2} / 4$ in opposite directions, being separated by $\ell$ at $t=0$ and recombine at $t=m \ell^{2} / 4$, after which they annihilate.

\footnotetext{
${ }^{21}$ The velocity approaches the speed of light as the ring size approaches the Compton scale. By this scale, the non-relativistic wavefunction description also breaks down.

${ }^{22}$ Note that this is again not the most general solution, we have chosen the factors so that the velocity flow around the vortices is circular.
} 


\section{Intersection and re-connection of vortices}

A natural question to ask about vortex lines is: what happens when they intersect? The generic behavior of the analytic solutions we have considered is for the vortices to re-connect whenever they come into contact, as opposed to becoming frustrated and forming a defect network. However, even arranging for vortex lines to intersect is somewhat non-trivial. The generic behavior seems instead to be for them to develop cusps that then meet at a point. As an example, we can consider the simplest solution that describes the collision of two vortex lines:

$$
\Psi=(x+i y)(y+i z)-\frac{t}{m} .
$$

At $t=0$ this describes two intersecting vortex lines oriented along the $z$ and $x$ axes. However, as a function of time, this solution describes two cusped vortices coming together at a point at $t=0$, which is indistinguishable at that moment from two infinitely long vortices intersecting. It is possible to construct more complicated configurations with intersecting vortices - for example solutions involving linked vortex rings - the fact that they intersect at a point and pinch off appears to be a generic feature.

\section{The random phase model and the number density of vortices}

Now that we have analyzed some generic features and convinced ourselves that vortices can appear, we would like to estimate the frequency of their occurrence. One might think with a random superposition of waves, the probability that chance interference takes $\Psi$ all the way to zero must be negligibly small. In fact - as we will verify using numerical simulations - such vortices (or defects) are ubiquitous: they exist with a number density of about one per de Broglie volume. In this section, we wish to gain some insight into this phenomenon by studying a simple analytic model - the random phase model. As a byproduct we will work out statistical properties of this model, facts which are useful beyond the immediate application to calculating defect number density - for instance, we will refer to these properties when we explore observational and experimental implications in Section 6. The random phase model is used in some of the axion detection literature, for example [85-87]; here we spell it out in detail and derive several statistical consequences.

\subsection{The random phase model}

In order to make progress, we need a stochastic model for $\Psi$. This model can be formulated in any number of dimensions. Suppose that $\Psi$ consists of a superposition of waves of different momenta, $\vec{k}$ :

$$
\Psi(\vec{x}, t)=\sum_{\vec{k}}\left(A_{\vec{k}} e^{i B_{\vec{k}}}\right) e^{i \vec{k} \cdot \vec{x}-i \omega_{k} t},
$$

where we have split the coefficients of the plane waves into an amplitude and a phase so that $A_{\vec{k}}$ and $B_{\vec{k}}$ are real. We have represented each mode as a plane wave; in a realistic halo, the mode functions might be more complicated and our model can be adapted accordingly (see footnote 24 below). In the same vein, $\omega_{k}$ is assumed to be $k^{2} /(2 m)$ for simplicity, but the dispersion relation can also be easily modified. 
It is helpful to split $\Psi$ into its real and imaginary parts as $\Psi=\Psi_{1}+i \Psi_{2}$, where the explicit expressions for its components are:

$$
\begin{aligned}
& \Psi_{1}(\vec{x}, t)=\frac{1}{2} \sum_{\vec{k}}\left(A_{\vec{k}} e^{i B_{\vec{k}}}\right) e^{i \vec{k} \cdot \vec{x}-i \omega_{k} t}+\left(A_{\vec{k}} e^{-i B_{\vec{k}}}\right) e^{-i \vec{k} \cdot \vec{x}+i \omega_{k} t} \\
& \Psi_{2}(\vec{x}, t)=\frac{1}{2 i} \sum_{\vec{k}}\left(A_{\vec{k}} e^{i B_{\vec{k}}}\right) e^{i \vec{k} \cdot \vec{x}-i \omega_{k} t}-\left(A_{\vec{k}} e^{-i B_{\vec{k}}}\right) e^{-i \vec{k} \cdot \vec{x}+i \omega_{k} t} .
\end{aligned}
$$

In order to streamline expressions, it is helpful to introduce a more compact notation:

$$
\Psi_{i}(\vec{x}, t)=\frac{1}{2} \sum_{\vec{k}}\left(A_{\vec{k}} e^{i b_{\vec{k}}^{i}}\right) e^{i \vec{k} \cdot \vec{x}-i \omega_{k} t}+\left(A_{\vec{k}} e^{-i b_{\vec{k}}^{i}}\right) e^{-i \vec{k} \cdot \vec{x}+i \omega_{k} t}
$$

where the index $i$ (to be distinguished from the imaginary number $i$ ) equals 1 or 2 , and

$$
b_{\vec{k}}^{i}= \begin{cases}B_{\vec{k}} & \text { if } i=1 \\ B_{\vec{k}}-\frac{\pi}{2} & \text { if } \quad i=2\end{cases}
$$

We are interested in the two-point function between all possible combinations of real and imaginary parts $\left\langle\Psi_{i}\left(\vec{x}_{1}, t_{1}\right) \Psi_{j}\left(\vec{x}_{2}, t_{2}\right)\right\rangle$. The statistical average can be done in two steps, first averaging over the phase $b_{\vec{k}}^{i}$, and then subsequently averaging over the amplitude $A_{\vec{k}}$. Note that $A_{\vec{k}}$ actually does not need to be stochastic at all. A simple, reasonable, model would be to have the a fixed $A_{\vec{k}}$. For instance, it can be a Gaussian, representing the distribution of momenta in an isothermal halo. In what follows, we will take this point of view, though it is a simple matter to take our expressions and average over $A_{\vec{k}}$, if one wishes $A_{\vec{k}}$ to be stochastically drawn from some distribution.

The key assumption of the random phase model is about the statistical properties of the phases of the coefficients of the Fourier modes. In particular, we have that

$$
\left\langle e^{i b_{\vec{k}}^{i}} e^{-i b_{\vec{k}^{\prime}}^{j}}\right\rangle=\delta_{\vec{k}, \vec{k}^{\prime}} \sigma_{i j} \quad \text { where } \quad \sigma_{11}=\sigma_{22}=1, \sigma_{12}=-\sigma_{21}=i .
$$

This holds if the phase, $B_{\vec{k}}$, for each Fourier mode is independently randomly drawn from a uniform distribution between 0 and $2 \pi$. One can think of this as the analog of populating a halo with stars, where the stellar orbits have random phases. With this understanding, the one-point function vanishes (as do all correlation functions involving an odd number of points). For the two-point function, it can be shown using (4.6) that

$$
\left\langle\Psi_{i}\left(\vec{x}_{1}, t_{1}\right) \Psi_{j}\left(\vec{x}_{2}, t_{2}\right)\right\rangle=\frac{1}{4} \sum_{\vec{k}} A_{\vec{k}}^{2}\left(\sigma_{i j} e^{i \vec{k} \cdot\left(\vec{x}_{1}-\vec{x}_{2}\right)-i \omega_{k}\left(t_{1}-t_{2}\right)}+\sigma_{j i} e^{-i \vec{k} \cdot\left(\vec{x}_{1}-\vec{x}_{2}\right)+i \omega_{k}\left(t_{1}-t_{2}\right)}\right) .
$$

It is worth noting that $\Psi_{1}$ and $\Psi_{2}$ are in general correlated. However, at equal time $t_{1}=t_{2}$, and assuming isotropy so that $A_{\vec{k}}=A_{-\vec{k}}$, they are uncorrelated. 
Using the two-point function (4.6), we can compute higher-point functions by Wick contraction. For example we have

$$
\left\langle e^{i b_{\vec{k}_{1}}^{i_{1}}} e^{i b_{\vec{k}_{2}}^{i_{2}}} e^{-i b_{\vec{k}_{3}}^{i_{3}}} e^{-i b_{\vec{k}_{4}}^{i_{4}}}\right\rangle=\delta_{\vec{k}_{1}, \vec{k}_{3}} \delta_{\vec{k}_{2}, \vec{k}_{4}} \sigma_{i_{1} i_{3}} \sigma_{i_{2} i_{4}}+\delta_{\vec{k}_{1}, \vec{k}_{4}} \delta_{\vec{k}_{2}, \vec{k}_{3}} \sigma_{i_{1} i_{4}} \sigma_{i_{2} i_{3}}
$$

Using this formula, we can likewise compute the four-point function of the real and imaginary parts of $\Psi$ :

$$
\begin{aligned}
\left\langle\Psi_{i_{1}}\left(\vec{x}_{1}, t_{1}\right) \Psi_{i_{2}}\left(\vec{x}_{2}, t_{2}\right) \Psi_{i_{3}}\left(\vec{x}_{3}, t_{3}\right) \Psi_{i_{4}}\left(\vec{x}_{4}, t_{4}\right)\right\rangle= & \left\langle\Psi_{i_{1}}\left(\vec{x}_{1}, t_{1}\right) \Psi_{i_{2}}\left(\vec{x}_{2}, t_{2}\right)\right\rangle\left\langle\Psi_{i_{3}}\left(\vec{x}_{3}, t_{3}\right) \Psi_{i_{4}}\left(\vec{x}_{4}, t_{4}\right)\right\rangle \\
& +(2 \leftrightarrow 3)+(2 \leftrightarrow 4) .
\end{aligned}
$$

This Wick contraction pattern continues to higher point correlation functions so that $\Psi_{1}$ and $\Psi_{2}$ are correlated Gaussian random fields. ${ }^{23}$

We can differentiate eq. (4.7) to obtain correlators between $\Psi$ and its derivatives. For our application below - computing the average number density of vortices or defects - we will be interested in correlations at coincident points, i.e., setting $\vec{x}_{1}=\vec{x}_{2}$ and $t_{1}=t_{2}$ (after taking derivatives) which leads to the correlations

$$
\begin{aligned}
& \left\langle\Psi_{i} \Psi_{j}\right\rangle=\delta_{i j} \frac{1}{2} \sum_{\vec{k}} A_{\vec{k}}^{2}, \\
& \left\langle\Psi_{i} \partial_{\mu} \Psi_{j}\right\rangle=\frac{1}{4} \sum_{\vec{k}} A_{\vec{k}}^{2} i k_{\mu}\left(-\sigma_{i j}+\sigma_{j i}\right), \\
& \left\langle\partial_{\nu} \Psi_{i} \partial_{\mu} \Psi_{j}\right\rangle=\delta_{i j} \frac{1}{2} \sum_{\vec{k}} A_{\vec{k}}^{2} k_{\nu} k_{\mu},
\end{aligned}
$$

where we have defined $k_{\mu}$ so that $k_{0}=-\omega_{k}$ and where its spatial components are $\vec{k} \cdot{ }^{24}$ If the distribution

\footnotetext{
${ }^{23}$ It is worth emphasizing that the random phase assumption alone is sufficient to guarantee $\Psi_{1}$ and $\Psi_{2}$ are Gaussian random fields. That is, only $B_{\vec{k}}$ needs to be stochastic. This might at first sight seem surprising: a real Gaussian random field in general requires Fourier coefficients that have random phases, and that have amplitudes which are stochastically distributed in a Gaussian fashion. In our model, the complex field $\Psi$ has Fourier amplitudes $A_{\vec{k}}$ which are not (or at least not necessarily) stochastic. It can be easily checked that its real and imaginary parts $\Psi_{1}$ and $\Psi_{2}$, do have stochastically distributed Fourier amplitudes, simply from the randomness of the phases $B_{\vec{k}}$.

${ }^{24}$ If we had used some mode functions other than the plane waves i.e., replacing eq. (4.1) by $\Psi(\vec{x}, t)=\sum_{\vec{k}} A_{\vec{k}} e^{i B_{\vec{k}}} f_{\vec{k}}(\vec{x}, t)$ where $f_{\vec{k}}$ 's are the appropriate mode functions for the halo in question and $\vec{k}$ is merely a label for them, essentially all the results go through with minimal modifications. For instance, the factor of $e^{i \vec{k} \cdot\left(\vec{x}_{1}-\vec{x}_{2}\right)-i \omega_{k}\left(t_{1}-t_{2}\right)}$ in eq. (4.7) is replaced by $f_{\vec{k}}\left(x_{1}\right) f_{\vec{k}}^{*}\left(x_{2}\right)$. For eq. (4.10), the right hand side for each line reads: $\delta_{i j} \sum_{\vec{k}} A_{\vec{k}}^{2}\left|f_{\vec{k}}\right|^{2} / 2, \sum_{\vec{k}} A_{\vec{k}}^{2}\left(\sigma_{i j} f_{\vec{k}} \partial_{\mu} f_{\vec{k}}^{*}+\sigma_{j i} f_{\vec{k}}^{*} \partial_{\mu} f_{\vec{k}}\right) / 4$, and $\sum_{\vec{k}} A_{\vec{k}}^{2}\left(\sigma_{i j} \partial_{\nu} f_{\vec{k}} \partial_{\mu} f_{\vec{k}}^{*}+\sigma_{j i} \partial_{\nu} f_{\vec{k}}^{*} \partial_{\mu} f_{\vec{k}}\right) / 4$, respectively. Note that in general, these expressions depend on space and time $\left(\vec{x}_{1}, t_{1}\right)$. Even in this more general context, eq. (4.9) remains correct.
} 
of momenta is isotropic so that $A_{\vec{k}}=A_{-\vec{k}}=A_{k}$, then (4.10) simplifies to:

$$
\begin{aligned}
& \left\langle\Psi_{i} \Psi_{j}\right\rangle=\delta_{i j} \frac{1}{2} \sum_{\vec{k}} A_{k}^{2}, \\
& \left\langle\Psi_{1} \partial_{t} \Psi_{2}\right\rangle=-\left\langle\Psi_{2} \partial_{t} \Psi_{1}\right\rangle=\frac{1}{2} \sum_{\vec{k}} A_{k}^{2} \omega_{k}, \\
& \left\langle\partial_{t} \Psi_{i} \partial_{t} \Psi_{j}\right\rangle=\delta_{i j} \frac{1}{2} \sum_{\vec{k}} A_{k}^{2} \omega_{k}^{2}, \\
& \left\langle\partial_{m} \Psi_{i} \partial_{n} \Psi_{j}\right\rangle=\delta_{i j} \delta_{m n} \frac{1}{2 d} \sum_{\vec{k}} A_{k}^{2} k^{2} .
\end{aligned}
$$

Here the simplifications occur because the positive and negative $\vec{k}$ terms in many of the sums cancel out.

As an example of how these quantities might be used, suppose we are interested in the joint probability distribution of $\Psi_{1}, \Psi_{2}$ and their spatial derivatives at a single point. Recalling that $\Psi_{1}$ and $\Psi_{2}$ are Gaussian random fields, the distribution is given by - assuming 2 spatial dimensions:

$$
P\left(\Psi_{i}, \partial_{j} \Psi_{k}\right)=\frac{1}{(2 \pi)^{3} \Gamma^{2} \tilde{\Gamma}^{4}} \exp \left(-\frac{\Psi_{1}^{2}+\Psi_{2}^{2}}{2 \Gamma^{2}}-\frac{\left(\partial_{1} \Psi_{1}\right)^{2}+\left(\partial_{2} \Psi_{1}\right)^{2}+\left(\partial_{1} \Psi_{2}\right)^{2}+\left(\partial_{2} \Psi_{2}\right)^{2}}{2 \tilde{\Gamma}^{2}}\right),
$$

where the quantities $\Gamma$ and $\tilde{\Gamma}$ are defined by:

$$
\Gamma^{2} \equiv \frac{1}{2} \sum_{\vec{k}} A_{k}^{2}, \quad \quad \tilde{\Gamma}^{2} \equiv \frac{1}{2 d} \sum_{\vec{k}} A_{k}^{2} k^{2} .
$$

Here $P\left(\Psi_{i}, \partial_{j} \Psi_{k}\right)$ is a shorthand for the 6-dimensional probability distribution

$$
P\left(\Psi_{i}, \partial_{j} \Psi_{k}\right) \equiv P\left(\Psi_{1}, \Psi_{2}, \partial_{1} \Psi_{1}, \partial_{2} \Psi_{1}, \partial_{1} \Psi_{2}, \partial_{2} \Psi_{2}\right)
$$

Note that this probability has has been properly normalized so that integrating it over its 6 arguments yields unity.

An even simpler application is to consider the probability distribution of $\Psi_{1}$ and $\Psi_{2}$ at a single point. That is, consider ${ }^{25}$

$$
\mathrm{d} \Psi_{1} \mathrm{~d} \Psi_{2} P\left(\Psi_{1}, \Psi_{2}\right)=\mathrm{d} \Psi_{1} \mathrm{~d} \Psi_{2} \frac{1}{2 \pi \Gamma^{2}} \exp \left(-\frac{\Psi_{1}^{2}+\Psi_{2}^{2}}{2 \Gamma^{2}}\right)
$$

If we rotate this to "polar coordinates" by defining $\Psi_{1}=|\Psi| \cos \theta$ and $\Psi_{2}=|\Psi| \sin \theta$, we see that the probability distribution is independent of $\theta$, and the probability distribution for $|\Psi|=\sqrt{\Psi_{1}^{2}+\Psi_{2}^{2}}$ is

$$
\mathrm{d}|\Psi| P(|\Psi|)=\mathrm{d}|\Psi| \frac{|\Psi|}{\Gamma^{2}} \exp \left(-\frac{|\Psi|^{2}}{2 \Gamma^{2}}\right) .
$$

\footnotetext{
${ }^{25}$ We use the same symbol $P$ to denote probability distribution in different contexts, so for example $P\left(\Psi_{1}, \Psi_{2}\right)$ should not be confused with $P\left(\Psi_{1}, \Psi_{2}, \partial_{1} \Psi_{1}, \partial_{2} \Psi_{1}, \partial_{1} \Psi_{2}, \partial_{2} \Psi_{2}\right)$, nor with $P(|\Psi|)$ below.
} 
The above reproduces the Rayleigh distribution for the field amplitude, discussed in [87]. In Section 5, we compare this distribution against the distribution measured from numerical simulations of haloes from gravitational collapse. We will see that this distribution captures the low density, or low $|\Psi|$, part well, but not the high density tail.

It is worth noting that with the distribution given by eq. (4.16), the expectation value of $|\Psi|$ is $\langle|\Psi|\rangle=$ $\sqrt{\pi} \Gamma / 2$, while its variance is given by $\left\langle|\Psi|^{2}\right\rangle=2 \Gamma^{2}$. Recalling that the dark matter density is given by $\rho=m|\Psi|^{2}$, we see that the probability distribution for density is exponential:

$$
\mathrm{d} \rho P(\rho)=\frac{\mathrm{d} \rho}{\bar{\rho}} e^{-\rho / \bar{\rho}},
$$

where $\bar{\rho}$ is the mean density $\bar{\rho}=\langle\rho\rangle=2 m \Gamma^{2}$. More generally, the higher moments of the distribution are given by $\left\langle\rho^{n}\right\rangle=\Gamma(n+1) \bar{\rho}^{n}$, where $\Gamma(n+1)$ is the Gamma function, not to be confused with $\Gamma$ above. ${ }^{26}$ The fact that the density distribution peaks at $\rho=0$ is a distinctive feature of destructive interference. The locations where $\rho$ vanishes are precisely the sites of the vortices we have been focusing on.

Direct detection experiments are typically sensitive to the real scalar field, $\phi$. It is straightforward to deduce the statistical properties of $\phi$ from the relation to $\Psi$ :

$$
\phi(\vec{x}, t)=\frac{1}{\sqrt{2 m}}\left(2 \Psi_{1}(\vec{x}, t) \cos (m t)+2 \Psi_{2}(\vec{x}, t) \sin (m t)\right),
$$

along with the facts that in the random phase model $\Psi_{1}$ and $\Psi_{2}$ are correlated Gaussian random fields with the correlation functions given by eq. (4.7). The amplitude of $\phi$ oscillations is essentially $|\Psi|=\sqrt{\Psi_{1}^{2}+\Psi_{2}^{2}}$. We will use this random phase model to explore implications for axion detection experiments in $\S 6$.

\subsection{The number density of vortices}

Before carrying out the computation of the average number of vortices or defects, let us discuss what this means in different number of spatial dimensions. Recall in one dimension (where the defects take the form of walls), it is not generic to have both the real and imaginary parts of $\Psi$ vanish at the same point; thus it is not useful to compute the average number density of defects in that case. In three spatial dimensions, the vanishing of both the real and imaginary parts of $\Psi$ typically occurs along a line, and since such a line should not end, generically one expects a vortex ring defect. It is therefore meaningful to ask what the number density of vortex rings is. However, working with an extended object like a ring is not so straightforward: computing the expected number density analytically involves considering the multiple-point probability distribution for $\Psi$, which is somewhat complex. The case of two spatial dimensions is special: here, the vortex or defect takes the form of a point, and it is possible to work out the average number density of such points by considering the one-point probability distribution for $\Psi$ and its derivatives - a problem we can solve exactly.

The number density of vortex defects can be expressed as

$$
n_{\mathrm{vortex}}(\vec{x})=\sum_{a} \delta\left(\vec{x}-\vec{x}_{a}\right)
$$

\footnotetext{
${ }^{26}$ For instance: $\Gamma(n+1)=n$ ! for integer $n, \Gamma(3 / 2)=\sqrt{\pi} / 2$ and so on.
} 
where $\vec{x}_{a}$ 's are the locations where $\Psi$ vanishes in some volume, and $\delta$ represents the Dirac delta function. If we are interested in the average number of vortex defects averaged over many realizations we can compute it schematically as [88]

$$
\left\langle n_{\text {vortex }}\right\rangle=\int \mathcal{D} \Psi(\vec{x}) \sum_{a} \delta\left(\vec{x}-\vec{x}_{a}\right) P(\Psi(\vec{x}))
$$

where $P(\Psi(\vec{x}))$ is the probability density for general field configurations. In order to actually evaluate this integral we have to make some simplifying assumptions, we Taylor expand $\Psi$ around its vanishing points $\vec{x}_{a}$, as

$$
\left.\Psi(\vec{x}) \simeq \vec{\nabla} \Psi\right|_{\vec{x}_{a}} \cdot\left(\vec{x}-\vec{x}_{a}\right) .
$$

We are interested in two spatial dimensions: let us use $x_{1}$ and $x_{2}$ to denote the two spatial coordinates (with $\partial_{1}$ and $\partial_{2}$ the respective derivatives). ${ }^{27}$ It is convenient to use $\Psi_{1}$ and $\Psi_{2}$ to denote the real and imaginary parts of $\Psi$ as we did earlier, so that $\Psi=\Psi_{1}+i \Psi_{2}$. Thus, $\vec{\nabla} \Psi$ can be thought of as a $2 \times 2$ matrix which can be inverted to express $\vec{x}-\vec{x}_{a}$ in terms of $\Psi$ and its derivatives. We can then change variables in the delta function to obtain

$$
\left\langle n_{\text {vortex }}\right\rangle=\int \mathrm{d}^{4}(\partial \Psi) \mathrm{d} \Psi|\operatorname{det} \vec{\nabla} \Psi| \delta(\Psi) P(\Psi, \partial \Psi) .
$$

We can write the average number density of point vortices in two dimensions in a slightly more explicit form as

$$
\left\langle n_{\text {vortex }}\right\rangle=\int \mathrm{d}^{4}(\partial \Psi)\left|\partial_{1} \Psi_{1} \partial_{2} \Psi_{2}-\partial_{2} \Psi_{1} \partial_{1} \Psi_{2}\right| P\left(\Psi_{1}=0, \Psi_{2}=0, \partial \Psi\right),
$$

where we use $\partial \Psi$ as a shorthand for the 4 combinations of derivatives of $\Psi_{1}$ and $\Psi_{2}$. The probability density $P$ is the 6-dimensional joint distribution for $\Psi_{1}, \Psi_{2}$ and $\partial \Psi$. Note that $P \mathrm{~d} \Psi_{1} \mathrm{~d} \Psi_{2} \mathrm{~d}^{4}(\partial \Psi)$ gives the probability for these 6 quantities taking the prescribed values at a single point in space.

The equation (4.23) is general, in the sense that it holds for any probability distribution $P$. To make progress, we need a statistical model for $\Psi$, and this is where we employ the random phase model. Plugging the expression for $P$ from eq. (4.12) into eq. (4.23), we find: ${ }^{28}$

$$
\left\langle n_{\text {vortex }}\right\rangle=\frac{1}{2 \pi} \frac{\tilde{\Gamma}^{2}}{\Gamma^{2}}
$$

where $\Gamma$ and $\tilde{\Gamma}$ are defined in eq. (4.13). Suppose $A_{k}$, which enters into the definitions for $\Gamma$ and $\tilde{\Gamma}$, takes the form $A_{k} \propto e^{-k^{2} / k_{0}^{2}}$. Replacing the summation over $\vec{k}$ by an integral, we arrive at the average density of vortices (i.e., number of zero crossings) in two dimensions: ${ }^{29}$

$$
\left\langle n_{\text {vortex }}\right\rangle=\frac{k_{0}^{2}}{8 \pi} .
$$

\footnotetext{
${ }^{27}$ Note we use the index in two distinct ways: the index $a$ of $\vec{x}_{a}$ (or 1 as in $\vec{x}_{1}$ in $\S 4.1$ ) labels which particular point we are interested in; the index $i$ of $x_{i}(i=1,2$ for two spatial dimensions) labels the spatial component.

${ }^{28}$ Evaluating this expectation value takes some work. The integral (4.23) can be done by rotating to polar coordinates in field space and using the Gaussian probability distribution for $\partial \Psi$ (4.12).

${ }^{29}$ In other words, we replace $\sum_{\vec{k}} \mapsto L^{2} /(2 \pi)^{2} \int \mathrm{d}^{2} k$ in two spatial dimensions, where $L$ is the infrared length scale so that $2 \pi / L$ is the fundamental mode. For three spatial dimensions, insisting $\langle\rho\rangle=m\left(\left\langle\Psi_{1}^{2}\right\rangle+\left\langle\Psi_{2}^{2}\right\rangle\right)=m \sum_{\vec{k}} A_{k}^{2}$ would mean $A_{k}=\sqrt{\langle\rho\rangle / m}\left(\sqrt{8 \pi} / L k_{0}\right)^{3 / 2} \exp \left(-k^{2} / k_{0}^{2}\right)$.
} 


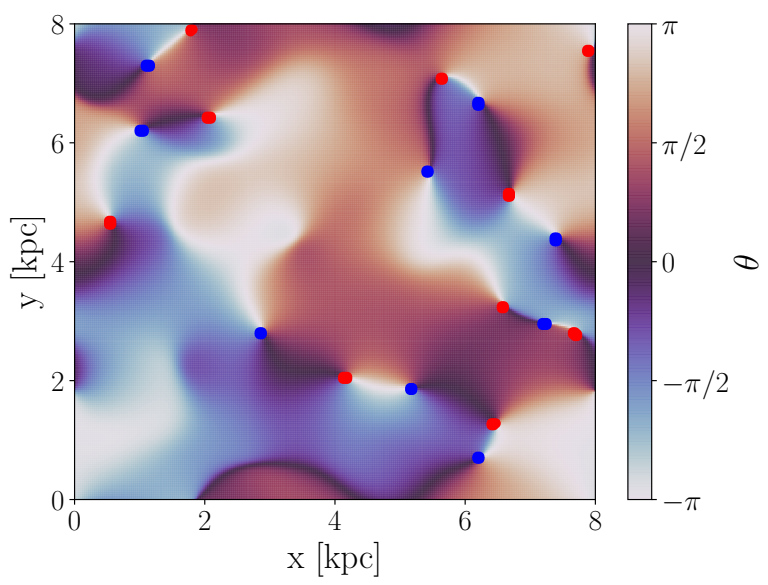

(a)

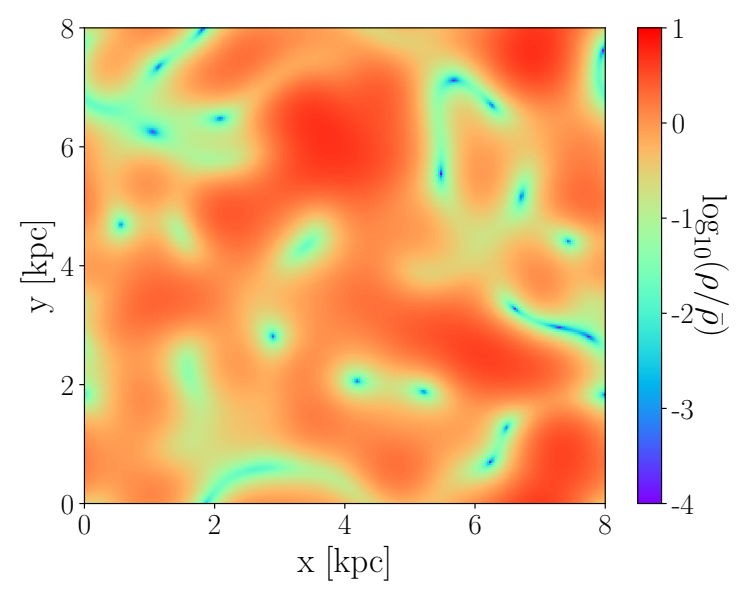

(b)

Figure 5: A snapshot of the phase (left panel) and density (right panel) from a numerical realization of the random phase model in two spatial dimensions. Here, $A_{\vec{k}}$ (see eq. (4.1)) is chosen to be $\propto e^{-k^{2} / k_{0}^{2}}$ with $k_{0}=2.63 \mathrm{kpc}^{-1}$. The corresponding $2 \mathrm{D}$ RMS momentum (weighted by $A_{\vec{k}}^{2}-$ i.e., mass weighting) is $k_{0} / \sqrt{2}$, and thus the de Broglie wavelength $\lambda_{\mathrm{dB}}=\sqrt{2} 2 \pi / k_{0}=3.38 \mathrm{kpc}$. On the left panel, the red and blue dots indicate vortices of opposite winding, that is, locations around which the phase winds by $2 \pi$ (clockwise for red, counter-clockwise for blue). On the right panel, the density normalized by the mean density $\bar{\rho}$ is shown on a logarithmic scale. It can be seen that the locations of vortices are also sites of very low density (consistent with zero), as expected.

Note that $k_{0}^{2} / 2$ is the momentum variance in 2 D. ${ }^{30}$ The associated de Broglie wavelength is $\lambda_{\mathrm{dB}}=\sqrt{2} 2 \pi / k_{0}$.

The average vortex density can be rewritten as $\left\langle n_{\text {vortex }}\right\rangle=\pi / \lambda_{\mathrm{dB}}^{2}$, which is roughly 3 per de Broglie area. As a check, we numerically generate realizations of the random phase model. A two-dimensional example is shown in Figure 5, where the red and blue dots highlight the vortices of opposite winding. All vortices have +1 or -1 winding - higher winding does not appear, consistent with our arguments in Section 2 . The analytic prediction for the expected number density of vortices is confirmed by these simulations.

We also generated three-dimensional realizations of the random phase model. Here, the vortices take the form of rings, and as discussed earlier, an analytic prediction of the average number density of rings is more challenging to work out, but we can measure it straightforwardly from the numerical realizations. The average number density of vortex rings comes out to be about $1.5 / \lambda_{\mathrm{dB}}^{3}$, where $\lambda_{\mathrm{dB}}$ is $2 \pi$ divided by the square root of the 3D momentum variance. An example is shown in Figure 6(a). The vortex identification algorithm is discussed in $\S 5$.

It is also possible to measure the size distribution of rings (defined as the maximum distance between

\footnotetext{
${ }^{30}$ In other words, $\int \mathrm{d}^{2} k k^{2} \exp \left(-2 k^{2} / k_{0}^{2}\right) / \int \mathrm{d}^{2} k \exp \left(-2 k^{2} / k_{0}^{2}\right)=k_{0}^{2} / 2$. This gives the momentum variance in twodimensions, and so the momentum variance in say the $x$-direction is $k_{0}^{2} / 4$. We square $A_{k}$ in computing this average because it is $|\Psi|^{2}$ that contributes to the mass density.
} 


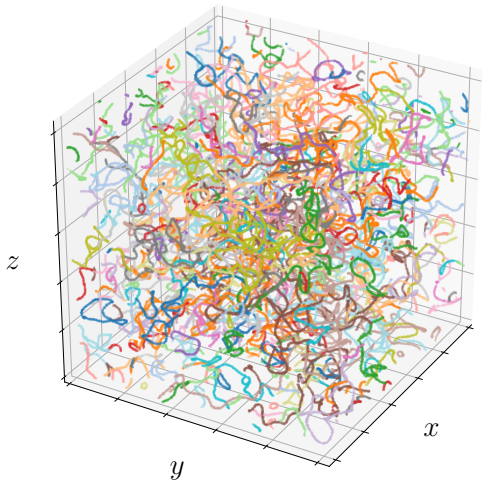

(a)

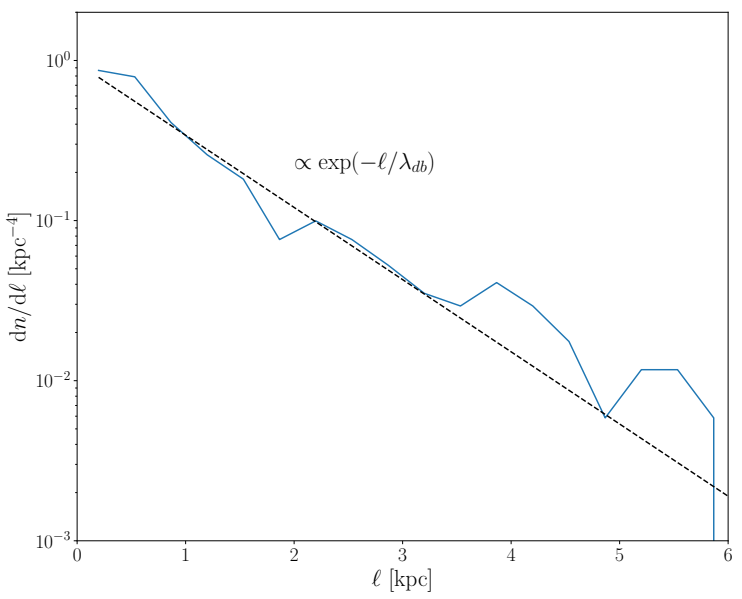

(b)

Figure 6: Numerical realizations of the random phase model in three dimensions. a. The lines highlight vortices in a three-dimensional simulation of the random phase model. All vortices take the form of vortex rings - note that cases that appear not to form a closed loop actually do once accounting for the periodic boundary condition. $\mathbf{b}$. Size distribution of vortex rings in a three-dimensional random phase simulation. The size of a ring is defined to be the maximum distance between two points on the ring. Here, the de Broglie wavelength $\lambda_{\mathrm{dB}}=1.15 \mathrm{kpc}$. The size distribution is approximately exponential.

two points on a ring) - this is shown in Figure 6(b). The distribution is roughly exponential:

$$
\frac{\mathrm{d} n_{\mathrm{vortex}}}{\mathrm{d} \ell} \sim \frac{1.5}{\lambda_{\mathrm{dB}}^{4}} e^{-\ell / \lambda_{\mathrm{dB}}}
$$

where $\ell$ is the size of a ring, and $n_{\text {vortex }}$ is the average number density of vortex rings. The fact that large rings are rare is not surprising: the real space correlation function of $\Psi$ is suppressed beyond the de Broglie scale. The fact that the distribution is roughly flat for small ring sizes might come as a surprise, given that the momentum distribution is suppressed at high $k$ 's. It should be kept in mind that ring size (or more precisely, the local ring curvature) is determined by a nonlinear combination of derivatives of $\Psi$.

\section{Vortex configurations in simulated haloes with gravity}

So far, our discussions of vortices have mostly been based on analytic solutions/models where the effects of gravity have been ignored. It is helpful to know to what extent our expectations about the behaviors of vortices hold up even when gravity is included. In this Section, we address this question using numerical simulations with gravity - essentially the analog of $N$-body simulations but for waves [32-34, 37, 54, 89-92]. Let us begin by summarizing a number of expectations based on the above discussion:

- We expect to find vortices in a collapsed halo which consists of a superposition of waves with different 
momenta. Such vortices form as a result of chance interference, and should exist even if the halo has no net angular momentum. The vortices should generically have winding \pm 1 , where the winding is given by (2.4).

- We expect vortices to come in the form of rings in three spatial dimensions, with a density of roughly one vortex ring per de Broglie volume. The de Broglie scale is $\lambda_{\mathrm{dB}}=2 \pi /(m v)$ where $v$ is the (three-dimensional) velocity dispersion of the halo.

- In the vicinity of vortices, the mass density should scale as $\rho \propto r_{\perp}^{2}$ where $r_{\perp}$ is the distance to the vortex as in (3.7), and the proportionality constant is roughly $\rho_{\text {local }} / \lambda_{\mathrm{dB}}^{2}$ with $\rho_{\text {local }}$ being the mean mass density of the halo in the neighborhood of the vortex, the idea being that the density near a vortex grows like $r_{\perp}^{2}$ until it reaches the local mean density, which happens approximately at the de Broglie wavelength. Further, we expect that the lines of constant density will be elliptical in the plane transverse to the vortex line as in (2.8).

- Near the vortices, the tangential (circulation) velocity is expected to scale inversely with distance to the vortex i.e., $v_{\|} \sim 1 /\left(m r_{\perp}\right)$.

- The vortex rings are dynamical objects: they can form and expand, or shrink and disappear, on a time scale of $\lambda_{\mathrm{dB}} / v$ or $2 \pi /\left(m v^{2}\right)$, where $v$ is the velocity dispersion of the halo. When vortices intersect, they reconnect as opposed to getting frustrated. Vortex rings are expected to move with a size-dependent velocity $v_{\text {ring }} \sim 1 /(m \ell)$ where $\ell$ is the size of the ring. More generally, a segment of a vortex is expected to move with the same scaling, with $\ell$ being the local curvature scale.

We confirm all of these expectations with our simulations, except the last bullet point, which requires examining simulation outputs at multiple times and will be left for future work. (See [78], for a study of some aspects of vortex dynamics.)

\subsection{Numerical algorithm}

Our numerical simulations are generated using the SPoS code presented and tested in [54]. The code solves the combined Schrödinger-Poisson system of equations in three spatial dimensions. Details of the initial conditions and parameters for different runs will be given below, but let us make a few general comments about them here. Cosmological simulations - meaning simulations that start from Gaussian random initial conditions, with a suitably large box (larger than $10 \mathrm{Mpc}$ co-moving) - do not quite have haloes that are sufficiently well-resolved for our purpose. We do observe vortex rings in such cosmological simulations [54], but in order to study the vortex rings in detail, we will instead focus on simulations with a small box size and somewhat artificial initial conditions chosen to result in a collapsed halo. In all cases we study, there are no vortices in the initial conditions. Yet, vortices generically appear after gravitational collapse brings about a superposition of waves in a virialized halo. This is the scenario we expect in the cosmological setting: the early universe is fairly homogeneous with small fluctuations (in particular $\Psi$ does not vanish anywhere in the early universe). Gravity amplifies the fluctuations and creates nonlinear objects in which a 
superposition of waves with suitably large amplitudes exist, making destructive interference, and therefore vortices, possible.

Given a simulation output, we identify vortex lines by searching for locations of high vorticity $\vec{\omega}=\vec{\nabla} \times \vec{v} .^{31}$ In order to do this, we first compute the normalized wavefunction $\tilde{\Psi} \equiv \Psi / \sqrt{\rho / m}=e^{i \theta}$. We then take the numerical gradient to compute the velocity $\vec{v}=\tilde{\Psi}^{*} \vec{\nabla} \tilde{\Psi} / i m$, and from that we can compute the vorticity field $\vec{\omega}$. Our numerical results show that $\vec{\omega}$ is close to zero in most regions, but has large values at the locations of vortex lines. One might imagine alternatively identifying vortices by searching for locations of zero density. Of course, numerically, the density is never strictly zero and we find that regions with low density sometimes also have small $\vec{\omega}$, indicating that small density alone is not a sufficiently robust indicator of vortex lines, and it is therefore better to search for regions of high vorticity.

After a vortex line is identified, we also check that the phase does have the required characteristic winding around it. This is important: given limited resolution, it might be difficult to ascertain that a particular point has low density or high vorticity, but the winding phase (or, equivalently, the non-zero velocity circulation) is something that can be checked in the neighborhood of that point and is therefore robust. The discrete grid points with large $\vec{\omega}$ are grouped into vortex lines using the DBSCAN (Density-Based Spatial Clustering of Applications with Noise) algorithm [93].

\subsection{Numerical solutions including gravity}

As a first example, we investigate a fairly symmetric configuration: a halo that forms from the coalescence of four evenly spaced density peaks. The three-dimensional computational box has a length of $L=0.5$ $\mathrm{Mpc}$ on each side. (In figures shown below, the origin is at the center of the box.) Note that our eventual conclusions - for instance about the abundance of vortices - can be scaled to whatever dark matter mass one is interested in, but for concreteness, we choose a rather low mass, $m=6 \times 10^{-24} \mathrm{eV}$. The relevant de Broglie scale is well-resolved with a simulation grid of $256^{3}$ points. We have also performed convergence tests with a higher resolution grid of $512^{3}$ points and found that our conclusions are not sensitive to the precise resolution.

The simulation is initialized from a symmetric configuration where four identical peaks are present on top of a background density of $\rho_{0}=1.88 \times 10^{-29} \mathrm{~g} / \mathrm{cm}^{3}$. The background density is chosen to resemble the cosmological one, even though there is no cosmological background expansion in the simulation. In fact, the precise value of $\rho_{0}$ does not have much impact on the eventual structure of the collapsed halo-its presence is primarily to ensure the density does not vanish anywhere in the initial condition. Each of the density peaks has the Gaussian profile

$$
\rho=250 \rho_{0} \exp \left[-\left(\frac{\vec{r}-\vec{r}_{c}}{r_{0}}\right)^{2}\right]
$$

where $r_{0}=0.05 L$ is the radius of the Gaussian core and $\vec{r}_{c}$ denotes the center of the peak. In the initial state, the four peaks are placed on the four vertices of a square chosen to lie in the $x=0$ plane, and

\footnotetext{
${ }^{31}$ Note that even though the fluid vorticity is expected to be zero away from vortices, there is always numerical noise (such as from taking gradients) that generates vorticity. Vortices are identified as places where the vorticity is exceptionally high.
} 


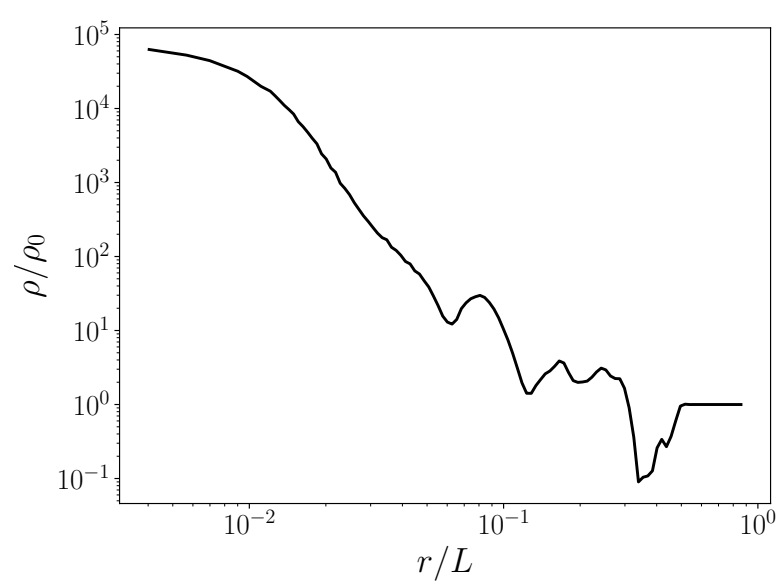

(a)

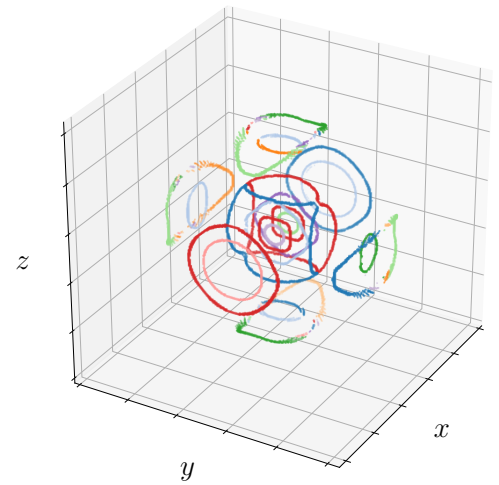

(b)

Figure 7: Numerical simulation of a halo formed from the collapse and merger of a symmetric configuration of four Gaussian peaks (see text for details). a. Spherically averaged density profile (as a function of radius) of the virialized halo that forms from the coalescence of the peaks. b. Vortex rings formed in the same halo.

which is centered at the origin with a side length of $L / 10$. The total mass in the computational box is $M=5.8 \times 10^{10} M_{\odot}$. The initial wavefunction is taken to be real $\Psi=\sqrt{\rho / m}$, so there is neither initial velocity nor angular momentum. We use an absorbing boundary condition to allow waves to leave the box. $^{32}$ Figure 7 (a) shows the density profile of the quasi-stationary dark matter halo that forms after the initial four peaks merge under the influence of gravity. A soliton core is formed in the inner region of size $r \sim 0.01 L$, and the density falls roughly as $r^{-3}$ in the outer region.

Figure 7(b) visualizes the vortex lines identified in the collapsed halo. The soliton core is rather smooth at the center, and vortices can be found outside the core. Around each vortex line the winding number is \pm 1 . All the vortex lines found are in the form of vortex rings. It is worth stressing that these vortex loops emerge from an initial configuration which does not have vorticity or angular momentum. By measuring the $3 \mathrm{D}$ velocity dispersion of the outer halo (away from the soliton core), we infer a de Broglie scale of $\lambda_{\mathrm{dB}} \equiv 2 \pi / m v \approx 0.12 L$. Note that the soliton core size of $\sim 0.01 L$ is in fact closer to $\lambda_{\mathrm{dB}} /(2 \pi)$ rather than $\lambda_{\mathrm{dB}}$. With a halo radius of about $0.2 L$, our one vortex ring per $\lambda_{\mathrm{dB}}^{3}$ estimate corresponds to an expected total of $\sim 19$ rings, in reasonable agreement with the 20 seen in the simulated halo.

Figure 8 shows two zoomed-in slices for the density and phase corresponding to the snapshot in Figure 7. The density slice plot on the left shows the $y z$ plane at $x=0.05 \mathrm{~L}$. (The symmetric initial condition

\footnotetext{
${ }^{32}$ This is accomplished by adding an extra term $-C\left(\Psi-\sqrt{\rho_{0} / m}\right)$ to the Schrödinger equation with $C=\arctan \left(10^{3} d / L\right) / \Delta t$ with $d=\max (0, r-0.48 L)$, where $r$ is the distance from the center of the box. This extra term is appreciable only in the region $r>0.48 L$ near the boundaries, removing fluctuations of $\Psi$ around the constant background. For the most of the box $r<0.48 L$, the evolution of $\Psi$ is essentially unaltered.
} 


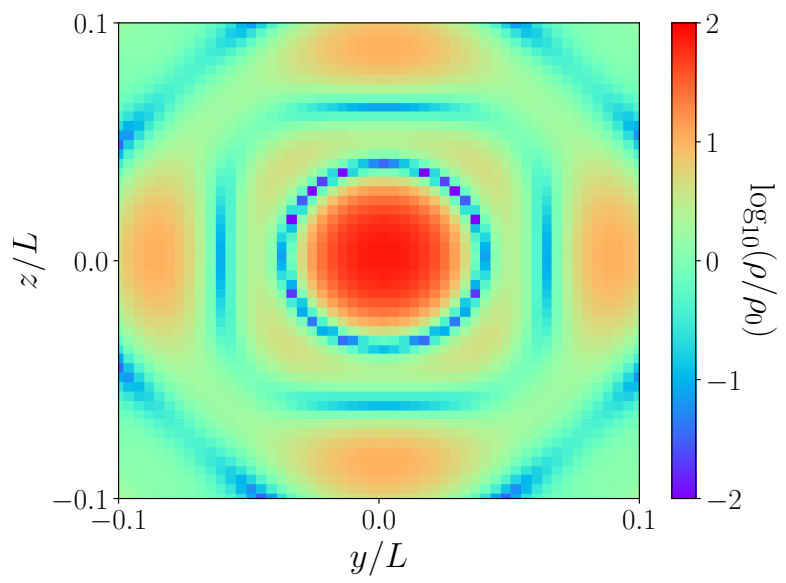

(a)

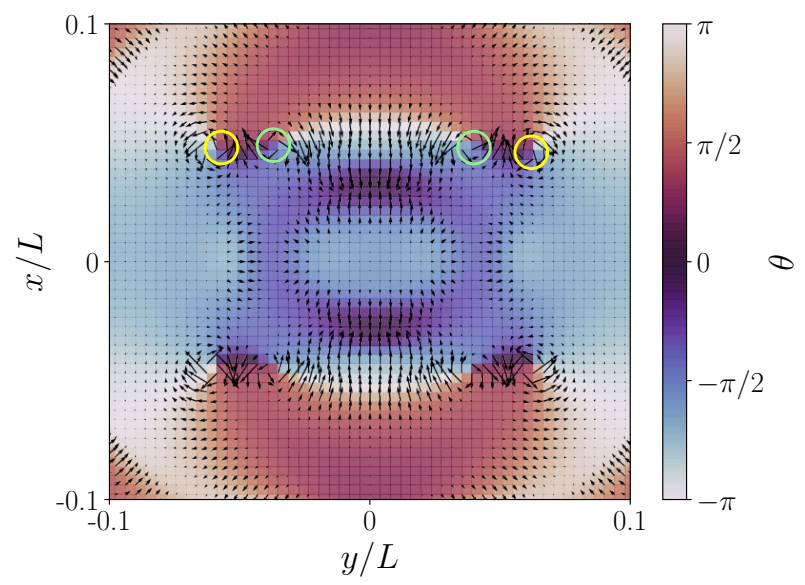

(b)

Figure 8: More detailed views of a halo depicted in Figure 7, formed from collapse of a symmetric configuration of four Gaussian peaks. a. A zoomed-in slice of the density field in the $y-z$ plane at $x=0.05 L$, showing vortex rings (where the density vanishes) observed near halo center. b. A zoomed-in slice of the phase $\theta$ in the $y x$ plane at $z=0$. The colors show the phase value and the arrows trace the velocity field in the plane. The green and yellow circles indicate where the circular and square vortex rings in the left panel intersect the $z=0$ plane. Note that there is a pair of such rings at $x=0.05 L$, and a pair at $x=-0.05 L$, due to the symmetric nature of the initial condition.

is such that the $x=-0.05 \mathrm{~L}$ plane looks more or less the same.) Near the center, there is a circle and a square loop of low density corresponding to the two vortex rings. The phase slice plot on the right shows the $y x$ plane at $z=0$, with arrows indicating the velocity field on that plane. The green and yellow circles show the position of the circular and square vortex rings as they intersect the $z=0$ plane (one pair at $x=0.05 L$ and one pair at $x=-0.05 L)$. The phase $\theta$ around each vortex smoothly varies from $-\pi$ to $\pi$, with winding number \pm 1 . Correspondingly, the velocity field wraps around each vortex line, as expected.

Figure 9 shows the density and velocity profiles close to four different vortices seen in Figure 7(b). Lines of the same color on the left and right correspond to the same vortex. Both the density and velocity profiles are cross-sectional, in the sense that they are profiles on a plane intersecting the vortex at some point, oriented perpendicular to the local vortex line direction. The (circularly averaged) density profile as a function of the distance from the vortex $r_{\perp}$ is shown on the left. As a comparison, we show in the same plot four dashed lines (color coded, one for each vortex) indicating $\rho=\rho_{\text {local }}\left(r_{\perp} / \lambda_{\mathrm{dB}}\right)^{2}$, where $\rho_{\text {local }}$ is the mean mass density at radii smaller than the location of the corresponding vortex. The $r_{\perp}^{2}$ profile is a reasonable approximation close to the vortex, but generally breaks down before $r_{\perp}$ reaches the de Broglie scale. The normalization by $\rho_{\text {local }}$ captures the qualitative trend that vortex rings closer to the halo center have a higher normalization, but can deviate from the actual normalization by an order of magnitude. The density bumps in the curves correspond to locations where one starts encountering the central region of the halo. The velocity profile is shown in Figure 9(b). It depicts the (circularly averaged) tangential 


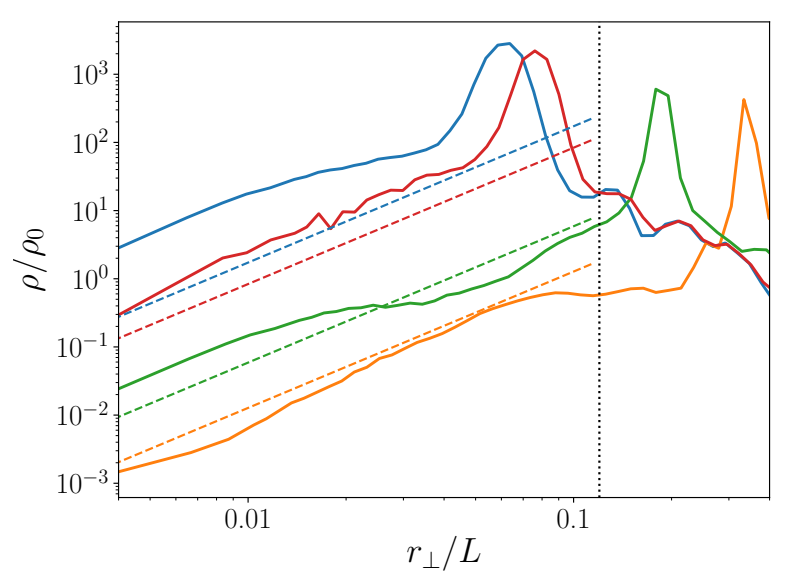

(a)

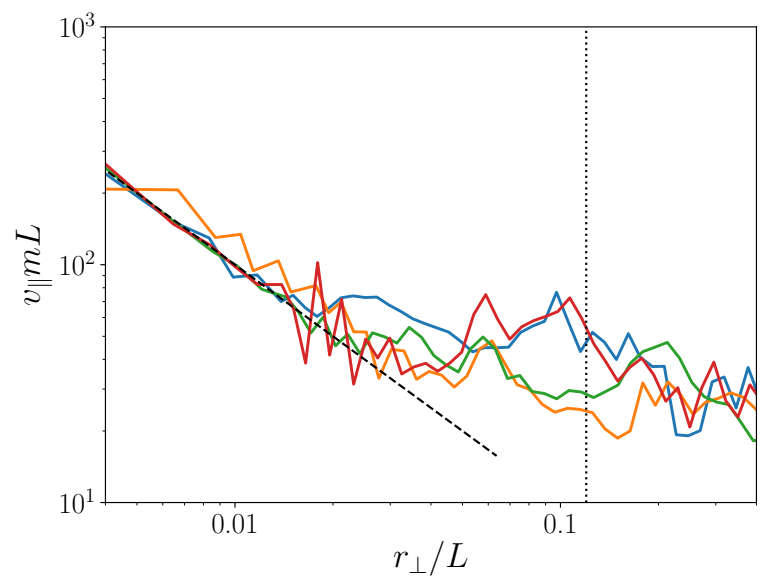

(b)

Figure 9: Density and velocity profiles around four vortices in Figure 7(b). The profiles are cross-sectional, in the sense that they depict (circularly averaged) profiles on a plane perpendicular to the vortex, intersecting the vortex at some point. a. Density profiles (solid lines) in the radial direction; the dashed lines indicate $\rho_{\text {local }}\left(r_{\perp} / \lambda_{\mathrm{dB}}\right)^{2}$, where $\rho_{\text {local }}$ is the mean density interior to the location of the vortex (see text), and $r_{\perp}$ is the distance to the vortex line. This shows that the near-vortex density profiles obey the expected $r_{\perp}^{2}$ scaling. b. Velocity profiles (solid lines); the black dashed line shows the tangential (circulation) velocity $v_{\|}=1 /\left(m r_{\perp}\right)$. The vertical dotted line in both panels shows the scale of $\lambda_{\mathrm{dB}}$. Lines of the same color on the left and right correspond to the same vortex.

velocity $v_{\|}$as a function of $r_{\perp}$. The black dashed line indicates $v_{\|}=1 /\left(m r_{\perp}\right)$ which describes the velocity profiles reasonably well.

The simulated halo discussed above arises from rather symmetric initial conditions, so one might be concerned that the formation of vortices and their properties are artifacts of these special initial conditions. As a second example, we therefore performed a simulation with more asymmetric initial conditions: there are initially 10 identical peaks (with profiles given by eq. (5.1)) randomly placed close to the center of the box (they are within $0.2 \mathrm{~L}$ of each other). The total mass in the computational box is kept the same. Figure 10 shows the density profile of the resulting halo from the coalescence of these peaks under gravity, with the vortex lines highlighted on the right. The vortex lines look less symmetric, but all qualitative features remain the same: (1) they all close in loops; $(2)$ the phase winds in the cross-section of each line with winding number \pm 1 ; (3) the density profile scales as $r_{\perp}^{2}$ and velocity profile scales as $1 / r_{\perp}$ close to the vortex; (4) the number density of vortex rings remains roughly one per de Broglie volume.

It's not surprising that these qualitative features should hold - they are expected on fairly general grounds with or without gravity (recall the Taylor expansion argument articulated in Section 2). The average number density of vortex rings is a quantity which in principle could be significantly modified when gravity is included, though it does not appear to be. Our computation in Section 4 is based on the random phase model, which raises the question: how good a description is this of a halo that forms from gravitational 


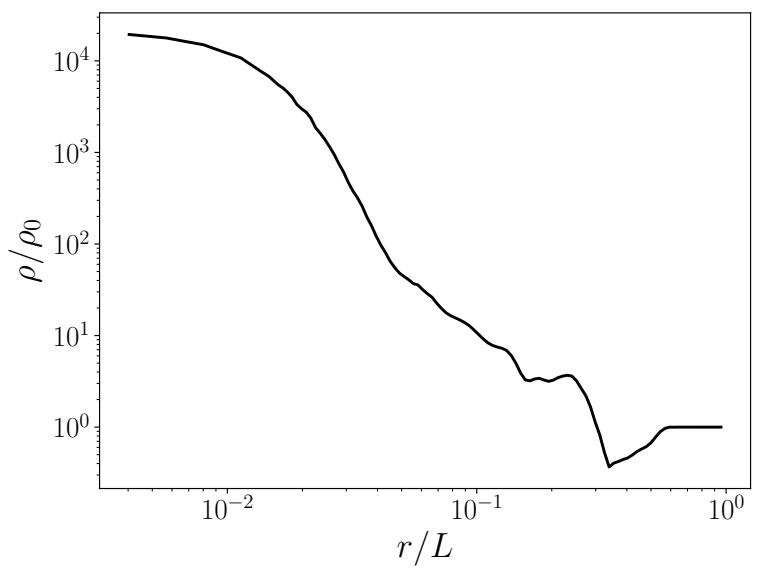

(a)

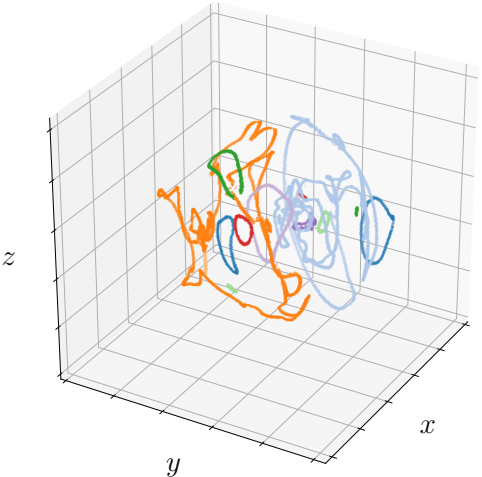

(b)

Figure 10: Features of a dark matter halo formed from collapse from asymmetric initial conditions (see text). a. Density profile of the halo. b. Visualization of vortex lines found in the halo.

collapse? As an indication, we show in Figure 11 the one-point probability distribution of density $\rho$ for the haloes depicted in Figure 7 and Figure 10, respectively. ${ }^{33}$ The solitonic core is excluded in measuring the probability distribution. In both cases, the distribution is reasonably approximated by the analytic prediction of the random phase model (eq. (4.17)), especially for small or moderate values of $\rho$ (see also eq. (4.16) for the corresponding distribution for $|\Psi|)$. The fact that the probability distribution $P(\rho)$ is exponential, and thus flat at small $\rho$, is a telltale sign of destructive interference. The random phase model prediction matches well the simulation results for the halo depicted in Figure 10, but less well for the halo in Figure 7. In the latter case, there appears to be some memory of the initial conditions, which are more symmetric; there is a prominent high $\rho$ tail, which is not captured by the random phase model. Some fraction of the halo substructure is in the form of sub-haloes - much like what one expects in conventional cold dark matter - and they contribute to this tail. We provide an approximate analytic fit to $P(\rho)$ in this case.

\section{Observational and experimental implications}

We have seen that dynamic small-scale structure is a generic feature of light dark matter. In this section, we discuss the observational and experimental implications of these interference substructures including, but not limited to, vortices. In order to organize our thinking, it is helpful to remind ourselves of certain

\footnotetext{
${ }^{33}$ The density $\rho$ of course has a systematic trend with radius i.e., overall, lower density at larger radii. We remove this systematic trend by considering $\rho / \bar{\rho}$, where $\bar{\rho}$ is the local mean density, estimated by averaging $\rho$ over spherical shells at the radii of interest. The probability distribution is then obtained from a histogram of $\rho / \bar{\rho}$.
} 

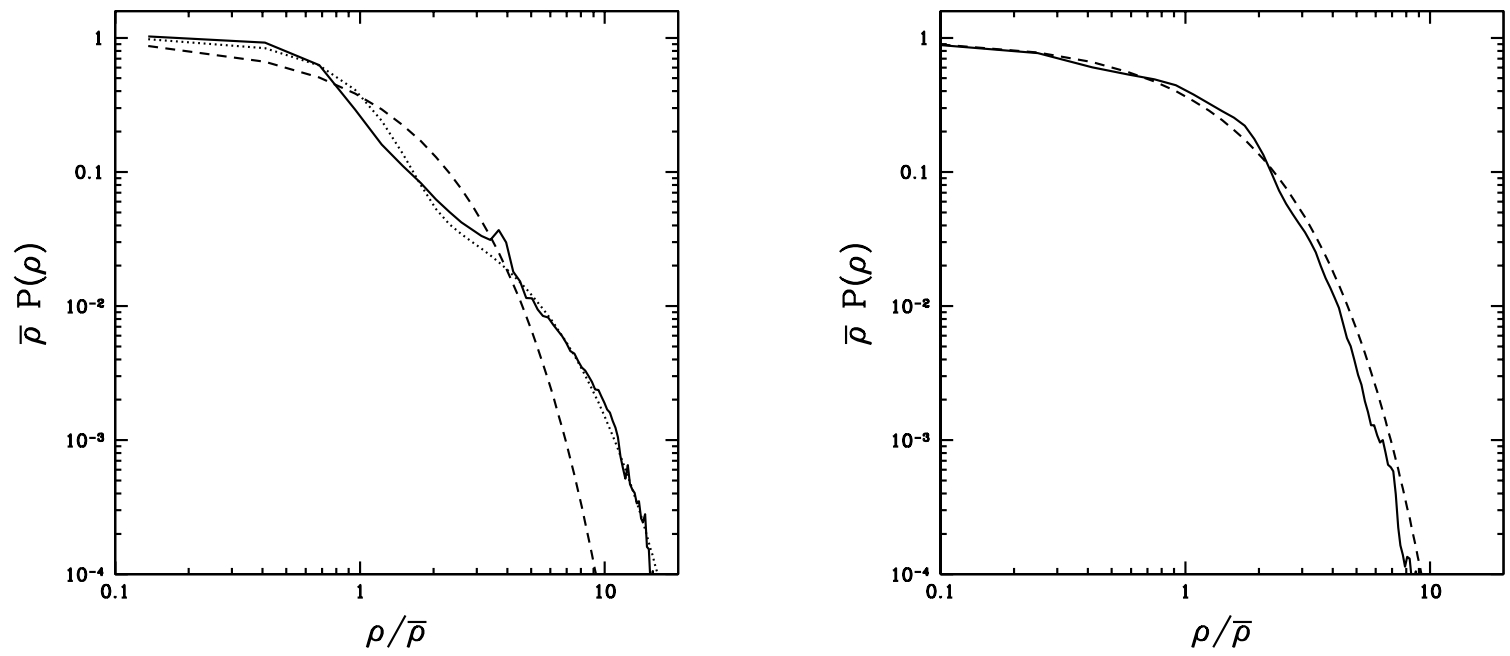

Figure 11: One-point probability distribution of density (solid lines) for haloes formed from gravitational collapse with symmetric (left panel) and asymmetric (right panel) initial conditions (see text). Here $P(\rho) \mathrm{d} \rho$ gives the probability that $\rho$ falls within the interval $\mathrm{d} \rho ; \bar{\rho}$ is the mean density. The dashed line in both panels denotes the random phase model prediction: $\bar{\rho} P(\rho)=e^{-\rho / \bar{\rho}}$ (eq. (4.17)). The dotted line in the left panel is an analytic approximation to the numerical data: $\bar{\rho} P(\rho)=0.9 e^{-1.06(\rho / \bar{\rho})^{2}}+0.1 e^{-0.42(\rho / \bar{\rho})}$.

characteristic length and time scales:

$$
\begin{aligned}
\lambda_{\mathrm{c}} & \equiv \frac{\pi}{m v}=0.24 \mathrm{kpc}\left(\frac{10^{-22} \mathrm{eV}}{m}\right)\left(\frac{250 \mathrm{~km} / \mathrm{s}}{v}\right)=7.4 \times 10^{4} \mathrm{~cm}\left(\frac{10^{-6} \mathrm{eV}}{m}\right)\left(\frac{250 \mathrm{~km} / \mathrm{s}}{v}\right), \\
t_{\mathrm{c}} & \equiv \frac{\lambda_{\mathrm{c}}}{v}=9.4 \times 10^{5} \mathrm{yr} .\left(\frac{10^{-22} \mathrm{eV}}{m}\right)\left(\frac{250 \mathrm{~km} / \mathrm{s}}{v}\right)^{2}=3 \times 10^{-3} \mathrm{~s}\left(\frac{10^{-6} \mathrm{eV}}{m}\right)\left(\frac{250 \mathrm{~km} / \mathrm{s}}{v}\right)^{2}, \\
\lambda_{\text {osc. }} & \equiv \frac{\pi}{m c}=0.2 \mathrm{pc}\left(\frac{10^{-22} \mathrm{eV}}{m}\right)=62 \mathrm{~cm}\left(\frac{10^{-6} \mathrm{eV}}{m}\right), \\
t_{\text {osc. }} & \equiv \frac{\lambda_{\text {osc. }}}{c}=0.6 \mathrm{yr} .\left(\frac{10^{-22} \mathrm{eV}}{m}\right)=2 \times 10^{-9} \mathrm{~s}\left(\frac{10^{-6} \mathrm{eV}}{m}\right),
\end{aligned}
$$

where we give baseline values both for the ultra-light extreme $\left(10^{-22} \mathrm{eV}\right)$ and the merely light, characteristic of QCD axion experiments $\left(10^{-6} \mathrm{eV}\right)$. The general features we discuss apply more broadly to a wide range of possible masses. We have restored the speed of light $c$ here for clarity, though $\hbar$ remains 1 . The velocity dispersion $v$ is chosen to be close to that of the Milky Way.

There is some freedom in the definition of the coherence length $\lambda_{\mathrm{c}}$ and time $t_{\mathrm{c}}$-for instance, we could have chosen to equate $\lambda_{\mathrm{c}}$ with the de Broglie scale $\lambda_{\mathrm{dB}}$, which is factor of 2 larger. The choice of $\lambda_{\mathrm{c}}$ is motivated by the random phase model discussed in Section 4: there the two-point function is given by $\left\langle\Psi_{1}(\vec{x}, t) \Psi_{1}(\vec{y}, t)\right\rangle /\left\langle\Psi_{1}^{2}\right\rangle=\exp \left(-k_{0}^{2}|\vec{x}-\vec{y}|^{2} / 8\right)$, and the coherence length $\lambda_{c}=\pi /\left(\sqrt{3} k_{0} / 2\right)$ is the 
separation at which the two-point function is suppressed by a factor of 0.2 , while at a separation of $\lambda_{\mathrm{dB}}$ the two-point function drops to $10^{-3} .{ }^{34}$ Note that the oscillation length and time scales, $\lambda_{\text {osc. }}$ and $t_{\text {osc. }}$, are related to the Compton (as opposed to de Broglie) scale - they are mostly relevant for axion direct detection experiments, whereas the characteristic scales $\lambda_{\mathrm{c}}$ and $t_{\mathrm{c}}$ are relevant for both astrophysical observations and detection experiments.

\subsection{Observational implications}

If the dark matter mass $m$ is in the ultra-light regime $\left(\lesssim 10^{-20} \mathrm{eV}\right.$, i.e., fuzzy dark matter), there are many astrophysically observable effects, some of which are detailed in [35]. Here, we wish to focus in particular on effects of the inevitable halo substructure due to wave interference. We concentrate mainly on gravitational lensing effects, that is the scattering of photons, and will briefly remark on the scattering of stars in Section 7.

\subsubsection{Lensing magnification}

We first consider the effects of light dark matter on lensing magnification in the context of strong lensing. We will show that the small-scale structure associated to interference generically leads to flux anomalies between images of order 10\%, making lensing a promising observational avenue to search for this substructure, including vortices.

At a basic level, gravitational lensing can be thought of as a mapping of the $2 \mathrm{D}$ plane to itself. The fundamental lensing equation takes the form (see e.g., the review [94]):

$$
\vec{\theta}_{S}=\vec{\theta}_{I}-2 \int_{0}^{r_{S}} \mathrm{~d} r_{L} \frac{r_{S}-r_{L}}{r_{S}} \vec{\nabla}_{\perp} \Phi
$$

where $\vec{\theta}_{S}$ and $\vec{\theta}_{I}$ are the $2 \mathrm{D}$ angular positions of the source and of the image in the sky, $\Phi$ is the (lens) gravitational potential, $r_{S}$ is the distance (from observer) to the source, $r_{L}$ is the distance (from observer) to the lens. The derivative $\vec{\nabla}_{\perp}$ is the spatial gradient perpendicular to the line of sight. ${ }^{35}$

We decompose $\Phi$ into two parts:

$$
\Phi=\Phi_{\text {smooth }}+\Phi_{\text {pert. }},
$$

where $\Phi_{\text {smooth }}$ represents the gravitational potential from a smooth halo or lens, and $\Phi_{\text {pert. }}$ is the perturbation from substructure, such as that due to wave interference.

Let us first study the effect of substructure on lensing magnification. The $2 \times 2$ lensing distortion matrix

\footnotetext{
${ }^{34}$ In the random phase model, the $3 \mathrm{D}$ momentum variance is $\int \mathrm{d}^{3} k k^{2} \exp \left(-2 k^{2} / k_{0}^{2}\right) / \int \mathrm{d}^{3} k \exp \left(-2 k^{2} / k_{0}^{2}\right)=3 k_{0}^{2} / 4$.

${ }^{35}$ The expression (6.2) holds even in an expanding universe with the metric $\mathrm{d} s^{2}=-\mathrm{d} t^{2}+a(t)^{2}\left(\mathrm{~d} r^{2}+r^{2} \mathrm{~d} \theta^{2}+r^{2} \sin { }^{2} \theta \mathrm{d} \varphi^{2}\right)$, with the understanding that $r_{S}$ and $r_{L}$ are co-moving distances and the gradient $\vec{\nabla}_{\perp}$ is also co-moving. In our discussion, we ignore the expanding background, and treat all distances as physical or proper. Note also our sign convention: the deflection angle for a point mass lens of mass $M$ and (physical) impact parameter $b$ is: $\vec{\alpha} \equiv-2 \int \mathrm{d} r_{L} \vec{\nabla} \perp \Phi=-(4 G M / b) \hat{b}$, where $\hat{b}$ is the unit vector pointing from lens to the point of closest approach of the photon.
} 
is

$$
\mathcal{A}_{i j} \equiv \frac{\partial \theta_{S}^{i}}{\partial \theta_{I}^{j}} \equiv\left(\begin{array}{cc}
1-\kappa-\gamma_{+} & -\gamma_{\times} \\
-\gamma_{\times} & 1-\kappa+\gamma_{+}
\end{array}\right)=\delta_{i j}-2 \int \mathrm{d} r_{L} \frac{\left(r_{S}-r_{L}\right) r_{L}}{r_{S}} \nabla_{\perp i} \nabla_{\perp j} \Phi,
$$

where the quantity $\kappa$ is known as convergence, and $\gamma_{+}$and $\gamma_{\times}$are the two components of the lensing shear [94]. It is the inverse of this matrix that dictates the mapping from source to image (in particular it is the Jacobian for this mapping). Note that the determinant $\left|\operatorname{det} \mathcal{A}^{-1}\right|$ gives the lensing magnification.

In most directions in the sky, $\kappa$ and $\gamma_{+, \times}$are small — at the percent level - and substructure introduces small corrections on top of that, which are likely difficult to observe. Occasionally, strong lensing occurs with multiple highly magnified images: in other words the determinant of $\mathcal{A}_{i j}$ is nearly zero. This provides an opportunity for the small contributions from substructure to make an observable difference. The idea that substructure introduces observable corrections to lensing magnification has been discussed by many authors (e.g, [95-105]). Most have focused on substructure in the form of sub-haloes in the parent lensing halo; here we are interested in substructure from wave interference.

Consider a location in the image plane where $\mathcal{A}_{i j}$ has a zero eigenvalue, let us call it the origin. Let us choose the orientation of axes such that $\gamma_{\times}=0$ at that point, and the zero eigenvalue is $1-\kappa-\gamma_{+}=0$. At the origin the lensing matrix therefore takes the form

$$
\mathcal{A}_{i j}(0)=\left(\begin{array}{cc}
0 & 0 \\
0 & 2\left(1-\kappa_{0}\right)
\end{array}\right)
$$

where $\kappa_{0}$ is the value of $\kappa$ at the origin. Generically, at the origin where $1-\kappa-\gamma_{+}$vanishes, both $\kappa$ and $\gamma_{+}$are of order unity so that $2\left(1-\kappa_{0}\right)$ is expected to be of order unity.

We are interested in images close to but not at the origin. Assuming for the moment that $\Phi$ arises entirely from $\Phi_{\text {smooth }}$, we can Taylor expand $\mathcal{A}_{i j}$ for $\vec{\theta}_{I}$ close to the origin:

$$
\mathcal{A}_{i j}\left(\vec{\theta}_{I}\right)=\left(\begin{array}{cc}
-\left.\vec{\nabla}\left(\kappa+\gamma_{+}\right)\right|_{0} \cdot \vec{\theta}_{I} & \mathcal{O}\left(\theta_{I}\right) \\
\mathcal{O}\left(\theta_{I}\right) & 2\left(1-\kappa_{0}\right)+\mathcal{O}\left(\theta_{I}\right)
\end{array}\right) .
$$

There is thus a line, the critical curve in the image plane ${ }^{36}$ defined by $\left.\vec{\nabla}\left(\kappa+\gamma_{+}\right)\right|_{0} \cdot \vec{\theta}_{I}=0$, where the magnification becomes very large. Suppose the origin in the image plane maps to/from some point in the source plane, say $\vec{\theta}_{S 0}$. It can be shown the structure of $\mathcal{A}_{i j}$ given in eq. (6.6) implies, for a source close to $\vec{\theta}_{S 0}$ :

$$
\begin{aligned}
& \theta_{S}^{x}-\theta_{S 0}^{x}=-\left.\frac{1}{2} \partial_{x}\left(\kappa+\gamma_{+}\right)\right|_{0} \theta_{I}^{x 2}+\mathcal{O}\left(\theta_{I}^{x} \theta_{I}^{y}, \theta_{I}^{y 2}\right), \\
& \theta_{S}^{y}-\theta_{S 0}^{y}=2\left(1-\kappa_{0}\right) \theta_{I}^{y}+\mathcal{O}\left(\theta_{I}^{x 2}, \theta_{I}^{y 2}, \theta_{I}^{x} \theta_{I}^{y}\right)
\end{aligned}
$$

where we have introduced $x$ and $y$ as coordinates to represent the components $i=1$ and $i=2$, for clarity. Assuming $\left.\partial_{x}\left(\kappa+\gamma_{+}\right)\right|_{0} \neq 0$, we see that $\theta_{I}^{x} \sim \pm \sqrt{-2\left(\theta_{S}^{x}-\theta_{S 0}^{x}\right) /\left.\partial_{x}\left(\kappa+\gamma_{+}\right)\right|_{0}}$ and $\theta_{I}^{y} \sim\left(\theta_{S}^{y}-\theta_{S 0}^{y}\right) /\left[2\left(1-\kappa_{0}\right)\right]$.

\footnotetext{
${ }^{36}$ Its counterpart in the source plane is known as the caustic. What we are describing here is the neighborhood of a fold caustic. See for example [106].
} 
Therefore, there are two images and they are equidistant from the origin. We can also see that if $\left(\theta_{S}^{x}-\theta_{S 0}^{x}\right)$ and $\left(\theta_{S}^{y}-\theta_{S 0}^{y}\right)$ are of the same order (and small), then $\theta_{I}^{y}$ is small in magnitude compared to $\theta_{I}^{x} \cdot{ }^{37}$ Therefore, if the source is on the correct side of the caustic, there are two images with magnification $|\mu|$, where the inverse magnification is given by the determinant of (6.6)

$$
\mu^{-1} \sim-\left.2\left(1-\kappa_{0}\right) \partial_{x}\left(\kappa+\gamma_{+}\right)\right|_{0} \theta_{I}^{x} \sim \pm 2\left(1-\kappa_{0}\right) \sqrt{2\left|\partial_{x}\left(\kappa+\gamma_{+}\right)\right|_{0}\left(\theta_{S}^{x}-\theta_{S 0}^{x}\right) \mid} \equiv \mu_{\text {smooth }}^{-1},
$$

where we define $\mu_{\text {smooth }}^{-1}$ for later convenience. This is the standard result that the lensing magnification $|\mu|$ scales inversely with distance from the critical curve in the image plane, or the square root of distance from the caustic in the source plane. Most relevant for our purpose: the two images have equal magnification $|\mu|$.

It is interesting that the above argument is similar in spirit to the argument for the generic properties of vortices in Section 2: both are based on a Taylor series expansion. ${ }^{38}$ The lensing argument is predicated upon the assumption that the gravitational potential $\Phi$ is smooth on the scale of the image separation. Adopting the split $\Phi=\Phi_{\text {smooth }}+\Phi_{\text {pert. }}$, perturbations on the scale of the image separation lead to a differential magnification between the two images. This allows us to use anomalous flux ratios to study halo substructure, as advocated by [95-102]. ${ }^{39}$

For us, the relevant substructure is the order unity fluctuations in density due to wave interference. The angular scale subtended by the corresponding coherence scale is:

$$
\theta_{\mathrm{c}} \equiv \frac{\lambda_{\mathrm{c}}}{r_{L}}=0.006 \operatorname{arcsec}\left(\frac{10^{-22} \mathrm{eV}}{m}\right)\left(\frac{1000 \mathrm{~km} / \mathrm{s}}{v}\right)\left(\frac{2 \mathrm{Gpc}}{r_{L}}\right)
$$

which is smaller than the typical image separation in systems with observable multiple images. We choose a velocity dispersion of $v \sim 1000 \mathrm{~km} / \mathrm{s}$ in order to correspond to a cluster scale lens. A galactic lens with $v$ of a few hundred $\mathrm{km} / \mathrm{s}$ would have a larger $\theta_{\mathrm{c}}$ but still smaller than the typical image separation. In order to model the effect of substructure from wave interference, suppose that $\Phi=\Phi_{\text {smooth }}+\Phi_{\text {pert. }}$. in the expression for $\mathcal{A}_{i j}$, eq. (6.4). The determinant of this matrix can then be written as

$$
\mu^{-1}=\mu_{\text {smooth }}^{-1}+\Delta \mu_{\text {pert. }}^{-1}
$$

where $\mu_{\text {smooth }}^{-1}$ is defined in eq. (6.9), and

$$
\Delta \mu_{\text {pert. }}^{-1} \equiv-4\left(1-\kappa_{0}\right) \int \mathrm{d} r_{L} \frac{\left(r_{S}-r_{L}\right) r_{L}}{r_{S}} \nabla_{\perp x} \nabla_{\perp x} \Phi_{\text {pert. }}
$$

\footnotetext{
${ }^{37}$ It can be checked this is a self-consistent solution, in that terms ignored in eq. (6.7) are $\mathcal{O}\left(\left[\theta_{S}-\theta_{S 0}\right]^{3 / 2}\right)$ or higher. The fact that $\theta_{I}^{x} \sim \pm \sqrt{-2\left(\theta_{S}^{x}-\theta_{S 0}^{x}\right) /\left.\partial_{x}\left(\kappa+\gamma_{+}\right)\right|_{0}}$ also implies that having two images requires $\theta_{S}^{x}$ to be on the correct side of the caustic so the quantity under the square root is positive.

${ }^{38}$ This type of argument is employed in catastrophe theory.

${ }^{39}$ It is important to distinguish between two different sources of differential magnification. The Taylor expansion argument tells us that two images close to a critical curve should have equal magnification to the lowest order. To the next order, assuming a smooth potential, the two images do differ slightly in magnification, fractionally at the level of $1 /\left|\mu_{\text {smooth }}\right|$. We are interested here in differential magnification caused by substructures i.e., perturbations to the smooth potential that introduce new scales, capable of enhancing the differential magnification beyond the minimal floor of $1 /\left|\mu_{\mathrm{smooth}}\right|$.
} 
where recall that $2\left(1-\kappa_{0}\right)$ is order unity. A quick way to estimate $\Delta \mu_{\text {pert. }}^{-1}$, which is likely an overestimate, is to note that wave interference leads to order unity density fluctuations on scale of $\lambda_{c}$. Adding up these fluctuations in a random walk fashion along the line of sight through the lensing halo produces

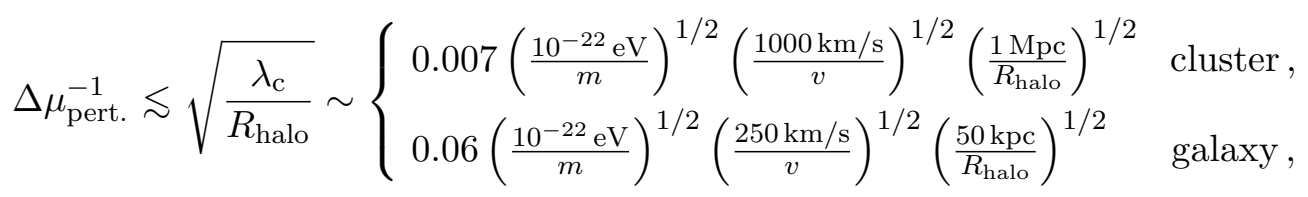

where $R_{\text {halo }}$ is the size of the lensing halo (so that there are $N=R_{\text {halo }} / \lambda_{\mathrm{c}}$ steps to the random walk, and we are estimating the average) and we give values for both a cluster and for a galaxy scale lens. This is an overestimate because most of the (smooth halo) contribution to the order unity $\kappa_{0}$ comes from the central region, where the supposed soliton resides, whereas the interference substructures are located in the outer halo where the density is lower.

As an alternative estimate, consider a halo of mean density $\bar{\rho}$ with $\nabla^{2} \Phi_{\text {pert. }}=4 \pi G \bar{\rho} \delta$ where $\delta$ is the density fluctuation. The root mean square (RMS) fluctuation for $\Delta \mu_{\text {pert. }}^{-1}$ can be computed using the Limber approximation:

$$
\left(\Delta \mu_{\text {pert. }}^{-1}\right)_{\mathrm{rms}}=16\left(1-\kappa_{0}\right) \pi G \bar{\rho} \frac{\left(r_{S}-r_{L}\right) r_{L}}{r_{S}} \sqrt{\frac{3 R_{\text {halo }}}{4} \int \frac{\mathrm{d}^{2} k_{\perp}}{(2 \pi)^{2}} P_{\delta}\left(k_{\perp}\right)},
$$

where $P_{\delta}$ is the $3 \mathrm{D}$ power spectrum of $\delta .40$ The momentum integral is convergent because fluctuations are smooth for $k_{\perp} \gtrsim \pi / \lambda_{\mathrm{c}}$. A reasonable estimate is that the integral is dominated by fluctuations on the characteristic scale $k_{\perp} \sim \pi / \lambda_{\mathrm{c}}$, so the integral is roughly $\lambda_{\mathrm{c}}$ (in other words, we assume $4 \pi k_{\perp}^{3} P_{\delta}\left(k_{\perp}\right) /(2 \pi)^{3}$ is order unity at the characteristic scale). Putting this together, we deduce

$$
\left(\Delta \mu_{\text {pert. }}^{-1}\right)_{\mathrm{rms}}=8\left(1-\kappa_{0}\right) \pi G \bar{\rho} \frac{\left(r_{S}-r_{L}\right) r_{L}}{r_{S}} \sqrt{3 R_{\text {halo }} \lambda_{\mathrm{c}}}=2\left(1-\kappa_{0}\right) 3^{3 / 2} v^{2} \frac{\left(r_{S}-r_{L}\right) r_{L}}{r_{S} R_{\text {halo }}} \sqrt{\frac{\lambda_{\mathrm{c}}}{R_{\text {halo }}}},
$$

where $v^{2}=G M_{\text {halo }} / R_{\text {halo }}=4 \pi G \bar{\rho} R_{\text {halo }}^{2} / 3$. A rough numerical estimate is therefore

$$
\left(\Delta \mu_{\text {pert. }}^{-1}\right)_{\mathrm{rms}} \sim \begin{cases}4 \times 10^{-4}\left(\frac{10^{-22} \mathrm{eV}}{m}\right)^{1 / 2}\left(\frac{v}{1000 \mathrm{~km} / \mathrm{s}}\right)^{3 / 2}\left(\frac{1 \mathrm{Mpc}}{R_{\mathrm{halo}}}\right)^{3 / 2} & \text { cluster }, \\ 4.5 \times 10^{-3}\left(\frac{10^{-22} \mathrm{eV}}{m}\right)^{1 / 2}\left(\frac{v}{250 \mathrm{~km} / \mathrm{s}}\right)^{3 / 2}\left(\frac{50 \mathrm{kpc}}{R_{\mathrm{halo}}}\right)^{3 / 2} & \text { galaxy },\end{cases}
$$

where we have assumed $2\left(1-\kappa_{0}\right) \sim 1$ and $\left(r_{S}-r_{L}\right) r_{L} / r_{S} \sim 1$ Gpc. Note that this estimate is about a factor of 10 smaller than (6.13).

In order to understand the impact of this substructure component, recall eq. (6.11): $\mu^{-1}=\mu_{\text {smooth }}^{-1}+$ $\Delta \mu_{\text {pert. }}^{-1}$ If substructure were absent, the two images close to the critical curve would have the same

\footnotetext{
${ }^{40}$ We have used the fact that $r_{L}$, the distance to the mean location of the lensing halo, is much larger than

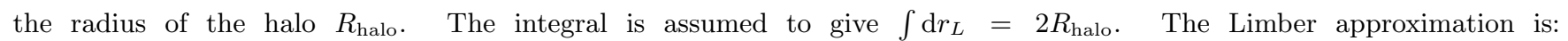
$\int \mathrm{d} r_{L}^{\prime} \int \mathrm{d} r_{L}\left\langle\nabla_{\perp x} \nabla_{\perp x} \Phi_{\text {pert. }}\left(r_{L}\right) \nabla_{\perp x} \nabla_{\perp x} \Phi_{\text {pert. }}\left(r_{L}^{\prime}\right)\right\rangle=2 R_{\text {halo }} \int \frac{\mathrm{d}^{2} k_{\perp}}{(2 \pi)^{2}} k_{\perp x}^{4} P_{\Phi_{\text {pert. }}}\left(k_{\perp}\right)$, where the power spectrum for $\Phi_{\text {pert. }}$ is $P_{\Phi_{\text {pert }} .}(k)=k^{-4}(4 \pi G \bar{\rho})^{2} P_{\delta}(k)$. The notation $P_{\Phi_{\text {pert }} .}\left(k_{\perp}\right)$ denotes $P_{\Phi_{\text {pert. }}}\left(k=k_{\perp}\right)$. In other words, the essence the Limber approximation is to use the line of sight integral to set the line of sight momentum $k_{\|}$to 0 .
} 
magnification $\left|\mu_{\text {smooth }}\right|$. Substructure makes $|\mu| \sim\left|\mu_{\text {smooth }}\right|\left(1-\mu_{\text {smooth }} \Delta \mu_{\text {pert. }}^{-1}\right)$, where $\Delta \mu_{\text {pert. }}^{-1}$ differs between the two images by an amount indicated by the RMS value estimated above. Thus, the flux anomaly (the departure from unity of the flux ratio between two images close to a critical curve) is

$$
\left|\mu_{\text {smooth }}\right| \Delta \mu_{\text {pert. }}^{-1} \sim \begin{cases}0.06\left(\frac{\left|\mu_{\text {smooth }}\right|}{150}\right)\left(\frac{\Delta \mu_{\text {pert. }}^{-1}}{4 \times 10^{-4}}\right) & \text { cluster } \\ 0.45\left(\frac{\left|\mu_{\text {smooth }}\right|}{100}\right)\left(\frac{\Delta \mu_{\text {pert. }}^{-1}}{4.5 \times 10^{-3}}\right) & \text { galaxy }\end{cases}
$$

where the values for $\left|\mu_{\text {smooth }}\right|$ correspond to those found for some cluster and galaxy lenses [98, 107]. If we had used the quick (over-)estimate for $\Delta \mu_{\text {pert. }}^{-1}$ derived earlier, the flux anomaly would be significantly bigger, e.g. $\sim 1$ for the cluster example. A recent paper based on simulations finds similar results to our conservative estimate [79]. It is also worth noting that a recent measurement of the flux anomaly in a cluster lens gives an anomaly at the level of tens of percent [107].

Two more comments before we leave this subject. (1) With more data, it would be possible to measure the flux anomaly as a function of image separation, in which case, one has effectively a means to measure the substructure power spectrum $[108,109]$. The interference substructure has a characteristic scale below which the power spectrum should be suppressed, which might provide a way to distinguish this kind of substructure from more conventional substructure due to sub-haloes. (2) In the random phase model, while the wavefunction $\Psi$ is Gaussian random, the density fluctuation is highly non-Gaussian. Thus computing the RMS density fluctuation does not quite capture the full picture of what is going on. In the language of vortices, we know from Fig. 6(b) there are vortices significantly smaller than the coherence scale $\lambda_{c}$. The associated steep spatial gradient could give enhanced lensing effects. This is worth further investigation.

\subsubsection{Lensing deflection}

A natural question to ask is whether there is an observable signal that probes specifically vortices or vortex rings (as opposed to generally probing small-scale interference). One distinctive feature of vortices is that they are associated with under-dense fluctuations, leading to de-magnification compared to the smooth background. Another interesting feature is that vortices can in principle move very fast (see eq. (2.12)): $v_{\text {vortex }} \sim 1 /(m R)$ where $R$ is either the size of a ring, or the curvature scale of a portion of a ring. Normally, in a given halo with velocity dispersion $v$, we would not consider objects moving at a much greater speed than $v$, because they would have escaped the halo. Vortices are an exception: $v_{\text {vortex }} \gg v$ is possible, because vortices can change and disappear before they could escape. Indeed, they are locations of zero density, so the contrast with their surrounding could give rise to interesting lensing signals. To exploit these features, we consider possible time-dependent observables.

The time varying flux of a distant source, due to gravitational lensing by a transiting vortex, is in principle an interesting signal - the flux would drop instead of increase during the transit. But the time scale of variation is very long. It is roughly $t_{\mathrm{c}} \sim 9 \times 10^{5} \mathrm{yr}$. for $v=250 \mathrm{~km} / \mathrm{s}$, or $\sim 5.6 \times 10^{4} \mathrm{yr}$. for $v=1000 \mathrm{~km} / \mathrm{s}$ (assuming $m=10^{-22} \mathrm{eV}$ ) (6.1). Consider for instance the flux anomaly deduced in the previous section (which is caused not by a single vortex but a random superposition of coherent patches): dividing the $10 \%$ 
anomaly by $t_{\mathrm{c}}$ gives an un-observably small variation per year.

Angular positions (i.e., astrometry) can be measured to much higher precision than fluxes. Is it possible to detect time varying lensing deflection? This was advocated by $[110,111]$ as a way to search for subhaloes inside the Milky Way halo. Sub-haloes can have much higher density than the average density of the parent halo, whereas the interference substructure gives rise to only order unity fluctuations in density, making it more challenging to detect. It is natural to ask if a high vortex velocity can make up for the shortfall.

Recall the setup: consider the lensing equation (6.2) with the gravitational potential split into $\Phi_{\text {smooth }}$ and $\Phi_{\text {pert. }}$. We are interested in $\Phi_{\text {pert. }}$ being sourced by the under-dense fluctuation associated with a moving vortex that happens to be crossing the line of sight to a distant source. The vortex resides in some galaxy along the line of sight - it could even be our own galaxy. Taking the time derivative of the lensing equation, and assuming $\dot{\theta}_{S}$ is negligible, such as for a distant source (an assumption we will revisit below), we obtain

$$
\dot{\theta}_{I} \sim 2 v_{\text {vortex }} \int \mathrm{d} r_{L} \frac{r_{S}-r_{L}}{r_{S}} \nabla_{\perp}^{2} \Phi_{\text {pert. }} .
$$

where we have made the estimate that $\dot{\Phi}_{\text {pert. }}$ is of order $v_{\text {vortex }}$ times a spatial derivative, and we are not keeping track of the vectorial nature of $\theta_{I}$ for simplicity. The gravitational potential $\Phi_{\text {pert. }}$ obeys the Poisson equation $\nabla^{2} \Phi_{\text {pert. }}=4 \pi G \delta \rho$, where $\delta \rho$ is the density fluctuation which we can model as

$$
\delta \rho=\left\{\begin{array}{ll}
\bar{\rho}\left(\frac{r_{\perp}^{2}}{r_{0}^{2}}-1\right) & \text { for } r_{\perp}<r_{0} \\
0 & \text { otherwise }
\end{array} .\right.
$$

The deflection from such an object can be worked out exactly (Appendix B) but for our purpose, dimensional analysis suffices (we ignore effects associated with relative orientation between vortex and line of sight). The time-variation of the deflection angle scales as

$$
\dot{\theta}_{I} \sim 8 \pi G \bar{\rho} r_{0} v_{\mathrm{vortex}} \frac{r_{S}-r_{L}}{r_{S}}
$$

where we assume $r_{0} \ll r_{L}$ ( $r_{L}$ being the location of the vortex lens).

There are two scenarios we can consider. A conservative scenario is to assume $v_{\text {vortex }}=1 /\left(m r_{0}\right)$, with $r_{0}$ signifying the size of the vortex ring in question. In this scenario, we see that the advantage from the high velocity of a small ring is completely washed out by the diminished effect from a small ring:

$$
\dot{\theta}_{I} \sim 4 \times 10^{-11} \operatorname{arcsec} / \mathrm{yr} .\left(\frac{\bar{\rho}}{0.3 \mathrm{GeV} \mathrm{cm}^{-3}}\right)\left(\frac{10^{-22} \mathrm{eV}}{m}\right)
$$

assuming $\left(r_{s}-r_{L}\right) / r_{s}$ is order unity. ${ }^{41}$ In this scenario - since small and large rings give similar contributionsthere is no reason to single out the deflection from a single vortex ring (or segment of a vortex ring). To

\footnotetext{
${ }^{41}$ Note that the location of the vortex lens does not matter much: it can be in a galaxy somewhere halfway between the source and us, or it can be located very close to us, including in our own galaxy. The factor of $r_{S}-r_{L} / r_{S}$ remains order unity, unless the lens is located very close to the source.
} 
estimate the net effect, one can therefore add up in a random walk fashion deflections from patches of size $\lambda_{\mathrm{c}}$ along the line of sight through a galaxy, similar to what we did for the magnification itself. Using $\sqrt{R_{\text {halo }} / \lambda_{\mathrm{c}}} \sim 20$, we find $\dot{\theta}_{I} \sim 8 \times 10^{-10} \operatorname{arcsec} / \mathrm{yr}^{42}$

Alternatively, an optimistic scenario goes like this: we have a vortex ring of size order $\lambda_{\mathrm{c}}$, but imagine there is a segment of it that has high curvature and is therefore moving at high speed (and crossing the line of sight to a distant source). The resulting estimate for the time variation of the lensing deflection is:

$$
\dot{\theta}_{I} \sim 5 \times 10^{-10} \operatorname{arcsec} / \mathrm{yr} .\left(\frac{\bar{\rho}}{0.3 \mathrm{GeV} \mathrm{cm}^{-3}}\right)\left(\frac{r_{0}}{0.24 \mathrm{kpc}}\right)\left(\frac{v_{\text {vortex }}}{10^{3} \mathrm{~km} / \mathrm{s}}\right) .
$$

The fact that this is roughly at the same level as the random walk estimate means both - deflection by a random sum of $\lambda_{c}$ patches and deflection by an especially high velocity vortex - should be taken into account. The estimate in eq. (6.22) might be overly optimistic: even though the vortex ring is of size $\lambda_{c}$, the high curvature around the segment of interest (so it has a high velocity) likely means the generic $r_{\perp}^{2}$ density profile extends to a smaller distance than $\lambda_{\mathrm{c}} \cdot{ }^{43}$ On the other hand, the density $\bar{\rho}$ could be quite a bit higher than the solar neighborhood value assumed here.

Even the optimistic $\dot{\theta}_{I}$ (6.22) is challenging to observe, for two reasons. One is technological: the Gaia experiment has a precision of about $10^{-6}$ arcsec at best, though this will improve with future experiments. The other challenge is proper motion: sources move so that $\dot{\theta}_{S} \neq 0$. Even a source at cosmological distance has $\dot{\theta}_{S} \sim 2 \times 10^{-8} \operatorname{arcsec} / \mathrm{yr}$., assuming source velocity of $200 \mathrm{~km} / \mathrm{s}$ and distance of $2 \mathrm{Gpc}$. One would therefore need a significantly higher density $\bar{\rho}$ for $\dot{\theta}_{I}$ to rise safely above the proper motion limit. It was suggested by [110-112] that if many sources were present, their correlated apparent motions due to lensing by an extended object (such as a vortex) can be used to look for very small effects. The expected $r_{\perp}^{2}$ density profile of a vortex, and its effective gravitational repulsion instead of attraction, might be helpful signposts in this endeavor.

\subsection{Experimental implications}

The small-scale features of light dark matter that we have been discussing also have implications for direct detection experiments. Different axion (or axion-like-particle ALP) detection experiments are sensitive to a range of masses: all the way from $10^{-15}-10^{-3} \mathrm{eV}[23,113]$. Interference substructures, including vortices, should be just as ubiquitous as they are in the ultra-light (i.e., fuzzy) regime. The only difference

\footnotetext{
${ }^{42}$ This estimate of the apparent image velocity does not crucially rely on details of the vortices; all it assumes is the existence of order unity density fluctuations on scale of $\lambda_{\mathrm{c}}$. It is also worth noting that the net image displacement after the passage of a single $\lambda_{\mathrm{c}}$ size blob or vortex is roughly $8 \pi G \bar{\rho} \lambda_{\mathrm{c}}^{2} \sim 10^{-4}$ arcsec assuming the same parameters as in eq. (6.21). The smallness of $\dot{\theta}_{I}$ is due to the fact that the passage takes such a long time.

${ }^{43}$ This is why we refrain from choosing an even higher $v_{\text {vortex }}$ in eq. (6.22). Note that a large vortex velocity (or for that matter large fluid velocity) is made possible by the nonlinear combination of derivatives of $\Psi$ that go into it (eq. (2.11)). Interesting observables typically involve multiplying this velocity by other quantities (such as density or gradient of density), which tends to counteract the effects of a large velocity.
} 
is the overall scale (see eq. (6.1)). Recall that the axion field $\phi$ is related to the wavefunction $\Psi$ by

$$
\begin{aligned}
\phi & =\frac{1}{\sqrt{2 m}}\left(\Psi e^{-i m t}+\Psi^{*} e^{i m t}\right) \\
& =\sqrt{\frac{2}{m}}\left(\Psi_{1} \cos (m t)+\Psi_{2} \sin (m t)\right) \\
& =\sqrt{\frac{2}{m}}|\Psi| \cos (m t-\theta),
\end{aligned}
$$

where we exhibit different ways to express $\Psi=\Psi_{1}+i \Psi_{2}=|\Psi| e^{i \theta}$ that will be helpful in our discussion.

The behavior of $\phi$ is governed by two different time scales. One is the fast oscillation time scale associated with $m$ (Compton): $t_{\text {osc. }} \sim 2 \times 10^{-9} \mathrm{~s}$ for $m \sim 10^{-6} \mathrm{eV}$. The other is a slower coherence time scale associated with $\Psi$ (de Broglie): $t_{\mathrm{c}} \sim 3 \times 10^{-3} \mathrm{~s}$ for $m \sim 10^{-6} \mathrm{eV}$. Moreover, $\Psi$ varies in space on the scale $\lambda_{\mathrm{c}} \sim 7.4 \times 10^{4} \mathrm{~cm}$. In other words, at a given location (say of the detector), the rapid $t_{\mathrm{osc}}$. oscillations of $\phi$ are modulated slowly on scale $t_{\mathrm{c}}$. The slow modulation is due to the interference substructures of interest. A recent paper that emphasizes this aspect of the signal is [87].

Why are interference substructures interesting for axion detection experiments? Let us give a brief preview: 1. At a minimum, they are relevant for properly interpreting experimental results: limits on the local axion density must account for the probability distribution of density [87]. 2. We will see that correlation functions of $\phi$ are expected to have definite shapes; the associated templates can be fruitfully applied to noisy data to enhance detection significance. 3. The probability distribution for $\phi$ contains information about the halo velocity dispersion which can be cross-checked against astronomical measurements. 4. Axion detection experiments largely focus on the amplitude $|\Psi|$ of axion oscillations. It is worth exploring the phase $\theta$ as another potential observable.

Two types of interactions are often used by axion detection experiments, which couple the axion either to photons or to fermions. ${ }^{44}$ The corresponding couplings are given by

$$
\mathcal{L}_{\text {int. }}^{\gamma} \sim g_{\gamma} \phi \epsilon_{\mu \nu \alpha \beta} F^{\mu \nu} F^{\alpha \beta}, \quad \mathcal{L}_{\text {int. }}^{\psi} \sim g_{\psi} \partial_{\mu} \phi \bar{\psi} \gamma^{\mu} \gamma_{5} \psi
$$

where $\phi$ is the axion, $F^{\mu \nu}$ is the photon field strength, $\psi$ is a fermion (for instance it can be the electron or a nucleon), $g_{\gamma}$ and $g_{\psi}$ are dimension-ful couplings, generically of the order of $1 / f$ where $f$ is the axion decay constant. Some general features of the physics of these couplings can be found in Appendices C and D. There are many axion detection experiments and it is beyond our scope to discuss them systematically (see [23, 25] for reviews). Here, we pick a few examples to illustrate the main points.

A cavity experiment such as ADMX [115] seeks photons produced in the presence of the axion and an external magnetic field, via the $\mathcal{L}_{\text {int. }}^{\gamma}$ interaction. The signal is often phrased in terms of the power output in a microwave cavity which is proportional to $\phi^{2}$, or $\phi^{2}$ averaged over the rapid oscillations [116]. Another type of experiment such as ABRACADABRA [117, 118] seeks a time varying magnetic field produced by an oscillating axion in the presence an external magnetic field. Its signal is proportional to $\dot{\phi}$. The typical

\footnotetext{
${ }^{44}$ There is also a possible coupling to gluons. See e.g., [114].
} 
size of these experiments is small compared to $\lambda_{c}$, thus one can think of the data stream as a time series of $\phi^{2}(t)$ or $\dot{\phi}(t)$ at a single location. The key point is that: $\phi$ is a stochastic variable because it consists of a superposition of waves. The expectation value $\left\langle\phi^{2}\right\rangle$ tells us the average dark matter density $\bar{\rho}=m^{2}\left\langle\phi^{2}\right\rangle$. In the random phase model, the probability distribution of $\rho$ is exponential $\propto e^{-\rho / \bar{\rho}}$ (see eq. (4.17)). Equivalently, the probability distribution for the amplitude of rapid oscillations of $\phi$ (essentially $|\Psi|$ ) obeys the Rayleigh distribution (see eq. (4.16)), as emphasized by [87]. In what follows, we will use the random phase model to explore several correlation functions involving $\phi$. But it is worth keeping in mind that while the random phase model captures the probability at low $|\Psi|$ well (destructive interference), it does not do a good job at high $|\Psi|$ in a halo formed from gravitational collapse, as evidenced by Figure 11. It would be useful to revisit the computation of [87], who pointed out that the constraints on the average local dark matter density are weakened in light of the Rayleigh distribution, largely because of the non-negligible probability of destructive interference. The high $|\Psi|$ tail above the Rayleigh distribution (as seen in Figure 11) acts in the opposite direction.

Recognizing that $\phi$ is a stochastic variable suggests new observables. One option is to measure the two point correlation function of $\phi:^{45}$

$$
\left\langle\phi(t) \phi\left(t^{\prime}\right)\right\rangle=\frac{1}{2 m}\left(\left\langle\Psi(t) \Psi^{*}\left(t^{\prime}\right)\right\rangle e^{-i m\left(t-t^{\prime}\right)}+\text { c.c. }\right),
$$

where c.c. represents complex conjugate. ${ }^{46}$ We assume $\left\langle\Psi(t) \Psi\left(t^{\prime}\right)\right\rangle=\left\langle\Psi^{*}(t) \Psi^{*}\left(t^{\prime}\right)\right\rangle=0$, consistent with (but more general than) the random phase model. ${ }^{47}$ Using the notation of Section 4 the $\phi$ two-point function can be rewritten in terms of real and imaginary parts $\Psi_{1}$ and $\Psi_{2}$ as

$$
\left\langle\phi(t) \phi\left(t^{\prime}\right)\right\rangle=\frac{2}{m}\left[\left\langle\Psi_{1}(t) \Psi_{1}\left(t^{\prime}\right)\right\rangle \cos \left(m\left(t-t^{\prime}\right)\right)+\left\langle\Psi_{2}(t) \Psi_{1}\left(t^{\prime}\right)\right\rangle \sin \left(m\left(t-t^{\prime}\right)\right)\right] .
$$

In the random phase model discussed in Section 4.1, the $\Psi_{i}$ correlation functions are given by

$$
\begin{aligned}
\left\langle\Psi_{1}(t) \Psi_{1}\left(t^{\prime}\right)\right\rangle & =\frac{1}{2} \sum_{\vec{k}} A_{\vec{k}}^{2} \cos \left[\omega_{k}\left(t-t^{\prime}\right)\right] \\
\left\langle\Psi_{2}(t) \Psi_{1}\left(t^{\prime}\right)\right\rangle & =-\frac{1}{2} \sum_{\vec{k}} A_{\vec{k}}^{2} \sin \left[\omega_{k}\left(t-t^{\prime}\right)\right], \\
\left\langle\Psi(t) \Psi^{*}\left(t^{\prime}\right)\right\rangle & =2\left\langle\Psi_{1}(t) \Psi_{1}\left(t^{\prime}\right)\right\rangle+2 i\left\langle\Psi_{2}(t) \Psi_{1}\left(t^{\prime}\right)\right\rangle .
\end{aligned}
$$

The $\Psi$ correlation functions are "slow" in the sense that they deviate from a constant when $t-t^{\prime}$ approaches the coherence time $t_{\mathrm{c}}$ or larger. The standard approach of considering output power $\left(\propto \phi^{2}\right)$ is essentially the same as focusing on equal times, $t=t^{\prime}$. For $t \neq t^{\prime}$, the oscillations on the timescale $1 / m$

\footnotetext{
${ }^{45}$ Here, we focus on correlators at a single spatial location but at two different times. More broadly, one could be interested in general space-time dependent correlators of the $\phi$ variables. We provide explicit formulas for these in Appendix E.

${ }^{46}$ The 2-point correlation function of $\dot{\phi}$ can be simply obtained by differentiation and making the non-relativistic approximation i.e. ignoring $\dot{\Psi}$ in comparison to $m \Psi,\left\langle\dot{\phi}(t) \dot{\phi}\left(t^{\prime}\right)\right\rangle=m^{2}\left\langle\phi(t) \phi\left(t^{\prime}\right)\right\rangle$.

${ }^{47}$ The assumption of $\left\langle\Psi(t) \Psi\left(t^{\prime}\right)\right\rangle=\left\langle\Psi^{*}(t) \Psi^{*}\left(t^{\prime}\right)\right\rangle=0$ is equivalent to $\left\langle\Psi_{1}(t) \Psi_{1}\left(t^{\prime}\right)\right\rangle=\left\langle\Psi_{2}(t) \Psi_{2}\left(t^{\prime}\right)\right\rangle$ and $\left\langle\Psi_{1}(t) \Psi_{2}\left(t^{\prime}\right)\right\rangle=$ $-\left\langle\Psi_{2}(t) \Psi_{1}\left(t^{\prime}\right)\right\rangle$. See eq. (4.7).
} 
provide a way to measure the axion mass. For large time separations $\left|t-t^{\prime}\right|$, the rapid oscillations would make it difficult to measure $\left\langle\phi(t) \phi\left(t^{\prime}\right)\right\rangle$. An alternative approach is to measure correlation functions of power itself. This correlation function also has pieces that oscillate with frequency $m$, but it has a piece that survives averaging over the rapid oscillations:

$$
\left\langle\phi(t)^{2} \phi\left(t^{\prime}\right)^{2}\right\rangle-\left\langle\phi^{2}\right\rangle^{2} \simeq \frac{1}{m^{2}}\left|\left\langle\Psi(t) \Psi^{*}\left(t^{\prime}\right)\right\rangle\right|^{2},
$$

where we assume $\Psi$ and $\Psi^{*}$ are correlated Gaussian random fields. ${ }^{48}$ This is a nice non-oscillatory quantity to consider, and the characteristic time separation on which it varies is the coherence time $t_{\mathrm{c}}$. This correlator can be computed exactly in the random phase model, assuming $A_{\vec{k}} \propto e^{-k^{2} / k_{0}^{2}}$, which leads to

$$
\xi\left(t-t^{\prime}\right) \equiv\left\langle\phi(t)^{2} \phi\left(t^{\prime}\right)^{2}\right\rangle-\left\langle\phi^{2}\right\rangle^{2} \simeq\left\langle\phi^{2}\right\rangle^{2}\left(1+\frac{k_{0}^{4}\left(t-t^{\prime}\right)^{2}}{16 m^{2}}\right)^{-3 / 2}=\frac{\bar{\rho}^{2}}{m^{4}}\left(1+\frac{\pi^{2}\left(t-t^{\prime}\right)^{2}}{9 t_{\mathrm{c}}^{2}}\right)^{-3 / 2} .
$$

The power correlation function, $\xi$, is also essentially the density correlation function. It is interesting that $\xi$ has a power law decay at large $\left|t-t^{\prime}\right|$ as opposed to an exponential one. Keep in mind that this is the unequal time correlation at a single location. The spatial correlation at equal time looks very different:

$$
\left\langle\phi(\vec{x})^{2} \phi\left(\vec{x}^{\prime}\right)^{2}\right\rangle-\left\langle\phi^{2}\right\rangle^{2} \simeq \frac{4}{m^{2}}\left\langle\Psi_{1}(\vec{x}) \Psi_{1}\left(\vec{x}^{\prime}\right)\right\rangle^{2}=\left\langle\phi^{2}\right\rangle^{2} \exp \left(-\frac{1}{4} k_{0}^{2}\left|\vec{x}-\vec{x}^{\prime}\right|^{2}\right),
$$

in particular it falls off exponentially with distance. The difference is due to the fact $\omega_{k}$, the frequency for a Fourier mode, goes as $k^{2}$ rather than $k$. In Appendix E for completeness we present the $\phi^{2}$ two-point function as a general space-time function, which reduces to (6.31) and (6.32) in the appropriate limits.

Axion detection experiments often have durations longer than $t_{\mathrm{c}}$, especially for the higher masses. It is thus possible to measure $\xi\left(t-t^{\prime}\right)$ for time separations exceeding $t_{\mathrm{c}}$. It is worth investigating to what extent the characteristic $\left|t-t^{\prime}\right|^{-3}$ decay can be exploited to help pull signal out of noise.

The axion-photon coupling is exploited in a different way in birefringence experiments, for instance ADBC [119]. There is no external magnetic field, and thus no photon creation. It seeks to detect, by interferometry, the phase difference between photons of opposite helicities propagating in an axion background. It can be shown the phase difference is proportional to $\phi(t, \vec{x})-\phi\left(t^{\prime}, \vec{x}^{\prime}\right)$, where $t, \vec{x}$ and $t^{\prime}, \vec{x}^{\prime}$ denote the beginning and end of the photon trajectory. This sets up the possibility, if suitable separations are chosen, to measure correlation functions in the full space-time sense (Appendix E), rather like interferometry in radio astronomy. Similar proposals were considered in [85, 120, 121].

So far, our discussion has been about general interference substructures and what they imply for axion detection. What about vortices in particular? Given that the typical size of experiments are much smaller than the de Broglie wavelength, the likelihood of encountering a vortex ring is small. Nonetheless, this doesn't preclude one passing close by, leading to a drop in the signal without going all the way to zero. Indeed, the fact that the one-point probability distribution of the density is exponential $\propto e^{-\rho / \bar{\rho}}(4.17)$

\footnotetext{
${ }^{48}$ Typically, the power is already an averaged quantity over the rapid oscillations i.e. $\phi^{2}(t)$ is implicitly averaged, in which case eq. (6.30) is exact. Note that the averaged output power is determined by $|\Psi|^{2}$ as well as the size of the external magnetic field and volume of the cavity.
} 
tells us destructive interference is not all that uncommon. It is also worth noting that if a vortex is actually encountered, it manifests as a phase jump (a wall) since the data stream in time is effectively one dimensional. To truly identify a vortex would require detectors at multiple locations to measure the telltale winding of the phase $\theta$ in the oscillation of the axion (6.23).

Another type of experiments make use of the coupling of the axion to fermions (such as nucleons) $\mathcal{L}_{\text {int. }}^{\psi}$. An example is CASPEr $[122,123]$. The coupling means the Hamiltonian for the nucleon has a term:

$$
H \sim g_{\psi} \vec{\nabla} \phi \cdot \vec{\sigma}
$$

where $\sigma$ is the nucleon spin operator. The concept is similar to nuclear magnetic resonance. Set up a magnetic field so the nucleon spin is aligned with it. The vector $\vec{\nabla} \phi$ (which oscillates with frequency $m$ ) is in general not aligned with the magnetic field, and causes the spin to precess. The effect is most pronounced when the strength of the magnetic field is such that the precession frequency matches $m$. Another type of experiment that utilizes the same coupling is the torsion pendulum experiment [124].

Our discussion of the correlation functions for $\phi$ and $\phi^{2}$ can be translated in a straightforward way to correlation functions for $\vec{\nabla} \phi$. One interesting feature of this technique is that encountering a vortex does not mean the signal goes to zero. At a vortex, $\phi$ vanishes, but $\vec{\nabla} \phi$ does not. This can be checked by studying the simple solution $\Psi \propto x+i y$ of a vortex extending in the $z$ direction (see Section 2 on why this is a good local approximation to a realistic vortex). This gives $\nabla_{x} \phi \propto \cos (m t)$ and $\nabla_{y} \phi \propto \sin (m t)$. This example also vividly shows that $\vec{\nabla} \phi$ is not necessarily described by $\sqrt{\bar{\rho}}$ times the velocity of the Earth through the dark matter halo. Wave interference inevitably causes space and time fluctuations in $\vec{\nabla} \phi$. The random phase model provides a useful starting point in characterizing them.

The existence of vortices tells us there is interesting spatial structure, i.e. winding, in the phase of $\Psi$. The phase manifests itself as the phase $\theta$ of axion oscillations: $\phi \propto|\Psi| \cos (m t-\theta)$. What observable can one use to access $\theta$ ? A possibility is:

$$
\phi \vec{\nabla} \dot{\phi}-\dot{\phi} \vec{\nabla} \phi=2|\Psi|^{2} \vec{\nabla} \theta
$$

where the equality holds in the non-relativistic regime $(|\dot{\Psi}| \ll m|\Psi|)$. This is essentially the fluid momentum density $\propto \rho \vec{v}$. It is not clear what the best way is to measure this quantity, or its correlation function. It could be a combination of experiments that use the axion-photon coupling and the axion-fermion coupling. It could also be a series of detectors in different locations. It would be interesting to explore whether this quantity can be fruitfully combined with other quantities to isolate $\vec{\nabla} \theta$, and under what conditions the phase winding is measurable. Perhaps there might be a way to connect this with some kind of Berry phase. We leave this for future work.

\section{Conclusions}

Dark matter consisting of particles lighter than about $\sim 30 \mathrm{eV}$ behaves essentially like a classical wave. Under the influence of gravity, haloes form with a central cored density profile surrounded by an an outer 
halo with a time-averaged density profile of the NFW form. Although the average density profile is similar to that of massive cold dark matter particles, the outer halo is a dynamical place: it can be thought of as a bound configuration of superposed waves interfering in a highly complex manner.

The classical superposition of wave-like dark matter in the outer halo region leads to a wide variety of interesting phenomena on small scales. In fact many of the interesting small-scale features that have been identified in models of ultra-light dark matter can be understood in this context. In this paper we have investigated these small-scale interference phenomena, focusing in particular on the formation of defects like vortex strings. The presence and dynamics of these objects are sufficiently generic that they can be characterized in a universal way.

We have investigated the formation and characteristics of vortices using a combination of analytical arguments and numerical simulations and have identified a number of robust features that wave-like dark matter vortices have: (1) The mere existence of vortices is generic in three spatial dimensions, where vorticity of the dark matter fluid is localized on closed loops where the dark matter density vanishes. (2) The number density of vortex rings is approximately one per de Broglie volume. (3) The winding (or velocity circulation) around such vortex lines is generically \pm 1 in units of $2 \pi / m$, where $m$ is the dark matter mass. (4) The dark matter density profile in the vicinity of the vortices scales like $\sim r_{\perp}^{2}$ (once circularly averaged) where $r_{\perp}$ is the distance from the vortex, while the (circularly averaged) velocity profile scales as $\sim 1 / r_{\perp}$. We further have argued for the following dynamical features of vortices: that rings should move with characteristic velocity inversely proportional to their size $v \sim(m \ell)^{-1}$ and that vortices should reconnect rather than frustrate. We have not verified these latter expectations in detail with numerics, and it would be very interesting to do so.

A natural question is how the existence of vortices might relate to angular momentum. The angular momentum of a halo is given by:

$$
\vec{J}=\int \mathrm{d}^{3} x \rho \vec{x} \times \vec{v}=-i \int \mathrm{d}^{3} x \vec{x} \times \Psi^{*} \vec{\nabla} \Psi
$$

assuming the origin is at the halo center of mass. The numerical examples in Section 5 show that even in a halo with zero net angular momentum, vortex rings appear anyway, as a result of chance interference. This suggests there is not a tight relation between halo angular momentum and the existence of vortices. Moreover, it is not difficult to construct solutions to the Schrödinger equation that carry angular momentum, but do not have vortices. Consider a superposition of the solutions discussed in (3.9). For example, adding together an $s$-wave and a solution of the form $\Psi_{l, l}$, we can arrange for a solution that globally carries $l$ units of angular momentum, but the wavefunction nowhere vanishes (by making the $s$-wave component large enough). This is not to say one cannot find solutions where angular momentum and vortices co-exist: for instance, a pure $\Psi_{l, l}$ solution on its own i.e., essentially an angular momentum eigenstate. ${ }^{49}$ Solutions of this kind that include gravity — rotating solitons - were investigated by [125]. Realistic haloes are mainly supported by velocity dispersion rather than rotation i.e. they are not described by an angular momentum eigenstate.

\footnotetext{
${ }^{49}$ By this we mean a field configuration $\Psi$ such that $\partial_{\varphi} \Psi \propto \Psi$, where $\varphi$ is the azimuthal angle, for angular momentum in the $z$ direction.
} 
Throughout this paper we have focused on the non-relativistic regime, where the dynamics is wellcaptured by the Schrödinger-Poisson equations. In this context, the winding around a vortex is a winding of the $\mathrm{U}(1)$ phase of the non-relativistic wavefunction. This corresponds to a winding in the phase space of the real scalar $\phi$. In the infrared, the total number of vortices is fixed once one imposes boundary conditions of fixed winding at infinity - vortices can only be produced in vortex-anti-vortex pairs, or as rings. It would be interesting to elucidate the properties of the symmetry that protects this number conservation and to investigate its fate in the ultraviolet.

The presence of small-scale features in general (and vortices in particular) in a dark matter halo can have a number of consequences for observations and experiments. We discussed both astrophysical signals for an ultra-light scalar (fuzzy dark matter) and experimental signals for a light, but not necessarily ultra-light, axion or axion-like particle. On the astrophysical side, a natural possibility is to detect the interferenceinduced $\mathcal{O}(1)$ density fluctuations via gravitational lensing. We estimated that interference substructures could induce $5-10 \%$ anomalous flux ratios in strongly lensed systems, in agreement with the recent study [79]. It would be useful to investigate in detail whether the $\sim 10 \%$ flux anomaly detected by [107] can be explained in this way, and how this explanation might be differentiated from one invoking small sub-haloes - such as by measuring the flux anomaly as a function of image separation. We took a first step in examining how the rapid motion of some vortices might impact on gravitational lensing. It would be useful to investigate further the motion of interference substructures in numerical simulations. This might have relevance beyond gravitational lensing. Stars in the galaxy can get heated by gravitational encounters with interference substructures [35, 126-129]. If some fraction of such encounters were at a high relative speed, as suggested by the example of vortices, is the heating efficiency thereby suppressed?

The wave-like nature of dark matter also has important consequences for local direct searches for axions or axion-like particles. The stochastic variability of the axion field suggests that density correlations either in time or space will be useful probes of models of this type. We advocate in particular the measurement of $\left\langle\phi^{2}(t) \phi^{2}\left(t^{\prime}\right)\right\rangle$ which has a characteristic $1 /\left|t-t^{\prime}\right|^{3}$ decay (6.31). It would be interesting to see if this feature can be exploited to extract a signal out of noisy data. We investigated how vortices impact experiments that exploit the axion-photon coupling versus those that use the axion-fermion coupling. It would be useful to improve upon the random phase model, to arrive at a more accurate description of the one-point probability distribution of the amplitude $|\Psi|$ of axion oscillations (Figure 11). More work needs to be done to investigate how to probe the phase of axion oscillations ( $\theta$ in (6.23)). Axion detection experiments largely focus on measuring the amplitude $|\Psi|$, for good reasons. The example of vortices illustrates there is interesting structure in the phase that can potentially be measured too. We hope to address these issues in the near future. 
Acknowledgements: We would like to thank Mustafa Amin, Matteo Biagetti, Greg Bryan, Andy Cohen, Liang Dai, Neal Dalal, Sergei Dubovsky, Benjamin Elder, Angelo Esposito, Matt Kleban, Andrey Kravtsov, Denis Martynov, Jerry Ostriker, Enrico Pajer, Alessandro Podo, Luca Santoni, Sergey Sibiryakov, Pierre Sikivie, Dam Son, John Stout, and Cora Uhlemann for helpful discussions. We wish to thank especially Alberto Nicolis for collaboration at an early stage, and Riccardo Rattazzi and Tanmay Vachaspati for raising questions that motivated this project. LH thanks Andy Cohen, Henry Tye, and the HKUST IAS for hospitality, and the Munich Institute for Astro- and Particle Physics (MIAPP) for a workshop where part of the research was done. Our work was supported in part by the NASA NXX16AB27G and the DOE DE-SC011941. AJ is supported in part by the Delta ITP consortium, a program of the Netherlands Organisation for Scientific Research (NWO) that is funded by the Dutch Ministry of Education, Culture and Science (OCW). XL thanks the Center for Computational Astrophysics at Flatiron Institute

for computational resources. Research at Perimeter Institute is supported in part by the Government of Canada through the Department of Innovation, Science and Economic Development Canada and by the Province of Ontario through the Ministry of Colleges and Universities. 


\section{A Solution-generating technique for the Schrödinger equation}

In [82] a useful procedure is given to produce solutions to the free Schrödinger equation with configurations of vortex lines. The basic idea is to take a solution to the time-dependent Schrödinger equation, $\Psi(\vec{x}, t)$ that at some initial time has the form of a plane wave times some spatial profile:

$$
\Psi(\vec{x}, 0)=e^{i \vec{k} \cdot \vec{x}} \phi(\vec{x})
$$

and from this construct a solution to the full Schrödinger equation. The key trick is to notice that $\Psi(\vec{x}, t)$ solves the Schrödinger equation for arbitrary $\vec{k}$, so we can differentiate $\Psi(\vec{x}, t)$ with respect to $\vec{k}$ arbitrarily to generate new solutions. At $t=0$ these solutions look like polynomials in $\vec{x}$ times $e^{i \vec{k} \cdot \vec{x}} \phi(\vec{x})$. Recall that vortices lie at the intersection between the places where the real and imaginary parts of the wavefunction vanish. So, by suitably choosing the differential operators, we can arrange for any configuration of vortex lines that can be described by the intersection of surfaces defined by polynomials. The time dependence of these configurations are then fixed by the solution $\Psi(\vec{x}, t)$.

All of the examples that we will need can be generated from the plane-wave solution to the Schrödinger equation:

$$
\Psi_{k}(\vec{x}, t)=e^{i \vec{k} \cdot \vec{x}-\frac{i \vec{k}^{2}}{2 m} t} .
$$

At $t=0$, this takes the form of a plane wave $\Psi(\vec{x}, 0)=e^{-\vec{k} \cdot \vec{x}}$. We can generate a configuration of vortex lines at $t=0$ by differentiating with respect to $\vec{k}$. If we then take the same differential operator and act on $\Psi(\vec{x}, t)$, this will generate a solution to the time-dependent Schrödinger equation which has the desired vortex configuration at $t=0$.

We may also be interested in situations with more than one vortex line. With gravity turned off, we are solving the free Schrödinger equation - which is linear - so superpositions will still solve the equation. But, they will typically not vanish anywhere, so blindly adding vortex solutions will generically remove the vortices. In order to get solutions with multiple vortices we have to be a little more careful. However, given two vortex configurations at $t=0$, their product will vanish at the locations of both of the vortices individually, and so will describe a configuration with two vortices. If we then take the differential operator that generates the $t=0$ configuration from (A.1) and act on (A.2) we can generate a time-dependent solution for the dynamics of this configuration. 


\section{B Gravitational lensing by a vortex}

In this appendix, we compute the deflection induced by a vortex with the density fluctuation profile considered in Section 6.1.2

$$
\delta \rho= \begin{cases}\bar{\rho}\left(\frac{r_{\perp}^{2}}{r_{0}^{2}}-1\right) & \text { for } r_{\perp}<r_{0} \\ 0 & \text { otherwise }\end{cases}
$$

with $\nabla^{2} \Phi_{\text {pert. }}=4 \pi G \delta \rho$. The lensing equation is

$$
\vec{\theta}_{S}=\vec{\theta}_{I}-2 \int_{0}^{r_{S}} \mathrm{~d} r_{L} \frac{r_{S}-r_{L}}{r_{S}} \vec{\nabla}_{\perp}\left(\Phi_{\text {smooth }}+\Phi_{\text {pert. }}\right)
$$

where $\vec{\theta}_{S}$ and $\vec{\theta}_{I}$ are are the source and image positions. Assuming a thin lens, we write this as

$$
\vec{\theta}_{S}=\vec{\theta}_{I}+\frac{r_{S}-r_{L}}{r_{S}} \vec{\alpha}_{\text {smooth }}+\frac{r_{S}-r_{L}}{r_{S}} \vec{\alpha}_{\text {pert. }}
$$

Suppose there is some source position that maps to some image position with $\vec{\alpha}_{\text {pert. }}$ switched off. The same source position then maps to a slightly different image position with $\vec{\alpha}_{\text {pert. }}$ turned on. Taking the difference between the corresponding lens equations, and relabeling $\vec{\theta}_{I}$ as the shift in image position, we have

$$
0=\vec{\theta}_{I}+\frac{r_{S}-r_{L}}{r_{S}} \vec{\alpha}_{\text {pert. }}+O\left(\theta_{I} \partial_{\theta_{I}} \alpha_{\text {smooth }}\right)
$$

where the term that involves derivative of $\alpha_{\text {smooth }}$ can be dropped assuming it is much less than unity.

The deflection $x$ component of $\vec{\alpha}_{\text {pert. is }}$

$$
\alpha_{\text {pert. }}=\operatorname{sgn}\left(\theta_{I}-\theta_{V}\right) 4 \pi G \bar{\rho} r_{0}^{2}\left\{\begin{array}{ll}
\frac{\pi}{2} & \text { if } b>r_{0} \\
\frac{\pi}{2}-\bar{\theta}+\frac{b^{2}}{r_{0}^{2}}\left(2 \bar{\theta}-\sin \bar{\theta}^{2}\right) & \text { otherwise }
\end{array},\right.
$$

where $b \equiv r_{L}\left|\theta_{I}-\theta_{V}\right|$ and $\cos \bar{\theta} \equiv b / r_{0}$. We have assumed $z$ is the line of sight direction, the vortex extends in the $y$ direction and $\theta_{V}$ represents its $x$ coordinate, $\vec{\theta}_{I}$ has vanishing $y$ component and $\theta_{I}$ exhibited above is its $x$ component. Substituting $\theta_{V}=v_{\text {vortex }} t / r_{L}$ allows one to deduce how $\theta_{I}$ changes with time $t$, for a vortex line that is moving in the $x$ direction (assuming $v_{\text {vortex }}>0$ ). For $t \rightarrow-\infty, \alpha_{\text {pert. }} \rightarrow 2 \pi^{2} G \bar{\rho} r_{0}^{2}$, which tells us $\theta_{I} \rightarrow-\left(r_{S}-r_{L}\right) 2 \pi^{2} G \bar{\rho} r_{0}^{2} / r_{S}$. The negative sign is correct, reflecting the effective repulsion of photons by the vortex (recall the source is at the origin). Conversely, for $t \rightarrow \infty, \alpha_{\text {pert. }} \rightarrow-2 \pi^{2} G \bar{\rho} r_{0}^{2}$, which tells us $\theta_{I} \rightarrow\left(r_{S}-r_{L}\right) 2 \pi^{2} G \bar{\rho} r_{0}^{2} / r_{S}$.

The vortex model used above could be made more realistic by allowing for a non-circularly symmetric density profile, and an arbitrary orientation of the vortex with respect to the line of sight and the direction of motion. Further, the vortex is generally part of a ring rather than an infinitely long string. 


\section{Axion-photon interaction}

Here, we summarize a few results relevant to our discussion in Section 6.2. A comprehensive review can be found in [25]. The Lagrangian for the axion and the photon (ignoring axion self-interaction) is

$$
\mathcal{L}=-\frac{1}{4} F_{\mu \nu} F^{\mu \nu}+A_{\mu} J^{\mu}-\frac{1}{2} \partial_{\mu} \phi \partial^{\mu} \phi-\frac{1}{2} m^{2} \phi^{2}+\frac{1}{4} g_{\gamma} \phi F_{\mu \nu} \tilde{F}^{\mu \nu}
$$

where $\tilde{F}^{\mu \nu} \equiv \epsilon^{\mu \nu \alpha \beta} F_{\alpha \beta} / 2$. The photon equation of motion is

$$
\partial_{\mu} F^{\mu \nu}=-J^{\nu}+g_{\gamma} \partial_{\mu}\left(\phi \tilde{F}^{\mu \nu}\right)
$$

which is equivalent to

$$
-\partial_{t} \vec{E}+\vec{\nabla} \times \vec{B}=\vec{J}-g_{\gamma} \partial_{t}(\phi \vec{B})-g_{\gamma} \vec{\nabla} \times(\phi \vec{E}) \quad, \quad \vec{\nabla} \cdot \vec{E}=J^{0}+g_{\gamma} \vec{\nabla} \cdot(\phi \vec{B}) .
$$

The setup for ADMX or ABRACADABRA, for instance, is to first generate some constant external magnetic field using $\vec{J}$, let's call it $\vec{B}_{0}$. With $J^{0}=0$, and assuming $\dot{\phi} \gg \nabla \phi$, we have

$$
-\partial_{t} \vec{E}+\vec{\nabla} \times \vec{B}=-g_{\gamma} \dot{\phi} \vec{B}_{0} \quad, \quad \vec{\nabla} \cdot \vec{E}=0 .
$$

The production of photons, or the generation of a magnetic field, can be deduced from these equations.

For photon propagation in an axion background (without an external magnetic field), eq. (C.3) can be used with $J^{\mu}$ set to zero. The standard relation between $\vec{E}$ and $\vec{B}$, following from $\partial_{t} \vec{B}+\vec{\nabla} \times \vec{E}=0$, continues to hold:

$$
\vec{\beta}=\frac{\vec{k}}{\omega} \times \vec{\epsilon}
$$

where $\vec{B}=\vec{\beta} e^{-i \omega t+i \vec{k} \cdot \vec{x}}$ and $\vec{E}=\vec{\epsilon} e^{-i \omega t+i \vec{k} \cdot \vec{x}}$. The wave equation is modified by the presence of the axion:

$$
\left(\partial_{t}^{2}-\nabla^{2}\right) \vec{E}=g_{\gamma}\left(\partial_{t} \phi \partial_{t} \vec{B}-\partial_{i} \phi \partial_{i} \vec{B}\right),
$$

and a similar equation holds with $\vec{E} \rightarrow \vec{B}$ and $\vec{B} \rightarrow-\vec{E}$. With the source turned off, $\vec{\epsilon}= \pm i \vec{\beta}$ gives the usual circular polarizations. Substituting this into eq. (C.6), one can deduce the modified dispersion relation to first order in $g_{\gamma}$ :

$$
\omega=|\vec{k}| \pm \frac{1}{2} g_{\gamma}(\dot{\phi}+\hat{k} \cdot \vec{\nabla} \phi) .
$$

Alternatively, a WKB analysis $\vec{E}=\vec{\epsilon} e^{i S}$ can be done to show the phase $S$ to first order in $g_{\gamma}$ is given by

$$
S=-|\vec{k}| t+\vec{k} \cdot \vec{x} \mp \frac{g_{\gamma}}{2} \int \mathrm{d} t \frac{D \phi}{D t},
$$

where $D / D t$ is a total time derivative: $\partial_{t}+\hat{k} \cdot \vec{\nabla}$. Thus the phase is sensitive only to $\phi$ at the beginning and at the end. Having a long path length does not confer an advantage except to make $\phi$ differ between start and finish. It is worth noting the various contributions to this difference: the dominant contribution is the Compton scale $m^{-1}$ time variation; the next contribution comes through the spatial variation of $\Psi$, giving the time scale $(m v)^{-1}$ (this is the de Broglie scale divided by the speed of light, relevant because we are interested in the propagation of photons); the smallest contribution comes through the time dependence of $\Psi$ with a time scale of $t_{\mathrm{c}} \sim\left(m v^{2}\right)^{-1}$. 


\section{Axion-fermion interaction}

We summarize a few results relevant for the discussion in Section 6.2. Comprehensive reviews can be found in $[23,25]$. Let us start with the interaction Hamiltonian associated with the coupling of an axion to the spin of a fermion such as some nucleus:

$$
H=-\mu B \sigma_{3}+g_{\psi} \vec{\nabla} \phi \cdot \vec{\sigma}
$$

where $B$ is an external magnetic field assumed to point in the $z$ direction, $\mu$ is the magnetic moment and $\vec{\sigma}$ represents the Pauli matrices. Assuming $B$ is large, we ignore the component of $\vec{\nabla} \phi$ in the $z$ direction, and keep only $\nabla_{x} \phi$ and $\nabla_{y} \phi$. Thus, the Hamiltonian takes the form

$$
H=\left(\begin{array}{cc}
-\mu B & g_{\psi}\left(\partial_{x} \phi-i \partial_{y} \phi\right) \\
g_{\psi}\left(\partial_{x} \phi+i \partial_{y} \phi\right) & \mu B
\end{array}\right)
$$

This can be rewritten as

$$
H=\left(\begin{array}{cc}
-\mu B & \eta e^{i m t}+\gamma e^{-i m t} \\
\eta^{*} e^{-i m t}+\gamma^{*} e^{i m t} & \mu B
\end{array}\right),
$$

where $\gamma \equiv g_{\psi}\left(\partial_{x} \Psi-i \partial_{y} \Psi\right) / \sqrt{2 m}$ and $\eta \equiv g_{\psi}\left(\partial_{x} \Psi^{*}-i \partial_{y} \Psi^{*}\right) / \sqrt{2 m}$. Assuming the state of the system is

$$
\left(\begin{array}{c}
e^{i m t / 2} g_{1}(t) \\
e^{-i m t / 2} g_{2}(t)
\end{array}\right)
$$

the Schrödinger equation takes the form

$$
i\left(\begin{array}{c}
\dot{g}_{1} \\
\dot{g}_{2}
\end{array}\right)=\left(\begin{array}{cc}
-\mu B+\frac{m}{2} & \eta \\
\eta^{*} & \mu B-\frac{m}{2}
\end{array}\right)\left(\begin{array}{l}
g_{1} \\
g_{2}
\end{array}\right)+\left(\begin{array}{cc}
0 & \gamma e^{-2 i m t} \\
\gamma^{*} e^{2 i m t} & 0
\end{array}\right)\left(\begin{array}{l}
g_{1} \\
g_{2}
\end{array}\right) .
$$

At or close to resonance, $\mu B=m / 2$, we can ignore the oscillatory second term on the right, and a general state evolves as

$$
\frac{1}{\sqrt{2}}\left(\begin{array}{c}
\left(c_{+} e^{-i|\eta| t}+c_{-} e^{i|\eta| t}\right) e^{+i m t / 2} \\
\left(c_{+} e^{-i|\eta| t}-c_{-} e^{i|\eta| t}\right) e^{-i m t / 2} \eta^{*} /|\eta|
\end{array}\right)
$$

where $c_{+}$and $c_{-}$are arbitrary constants. For illustration, choosing $c_{+}=c_{-}=1 / \sqrt{2}$ (corresponding to spin up at $t=0)$, we find

$$
\left\langle\sigma_{3}\right\rangle=\cos (2|\eta| t) \quad, \quad\left\langle\sigma_{1}\right\rangle=-\sin (2|\eta| t) \sin \left(m t+\theta_{\eta}\right) \quad, \quad\left\langle\sigma_{2}\right\rangle=-\sin (2|\eta| t) \cos \left(m t+\theta_{\eta}\right)
$$

where $\eta \equiv|\eta| e^{i \theta_{\eta}}$. Thus, measuring the $(x, y$ and $z)$ magnetization from a set of spins provides information on $\eta$, related to a particular combination of derivatives on $\Psi$. 


\section{E Real scalar correlations from the random phase model}

Here we collect some formulas that are useful in the analysis presented in Section 6.2. The observable quantities of interest are local correlation functions for the real scalar field, $\phi$, which is related to the non-relativistic wavefunction through the formula

$$
\phi(\vec{x}, t)=\frac{1}{\sqrt{2 m}}\left(\Psi(\vec{x}, t) e^{-i m t}+\Psi^{*}(\vec{x}, t) e^{i m t}\right) .
$$

Axion direct detection experiments are sensitive to correlation functions of operators built out of $\phi$. In order to characterize the statistics of these fluctuations, we employ the random phase model introduced in Section 4. The key equation we require from the random phase model is the two-point correlation function

$$
\left\langle\Psi^{*}(\vec{x}, t) \Psi\left(\vec{y}, t^{\prime}\right)\right\rangle=\sum A_{k}^{2} \exp \left(i \vec{k} \cdot(\vec{x}-\vec{y})-i \omega_{k}\left(t-t^{\prime}\right)\right)
$$

Assuming a dispersion relation $\omega_{k}=k^{2} / 2 m$ and assuming a statistical distribution for $A_{k}$ :

$$
A_{k} \propto e^{-k^{2} / k_{0}^{2}}
$$

we can approximate the sum in (E.2) by an integral to obtain

$$
\left\langle\Psi^{*}(\vec{x}, t) \Psi\left(\vec{y}, t^{\prime}\right)\right\rangle \propto k_{0}^{3}\left(1+\frac{i k_{0}^{2}\left(t-t^{\prime}\right)}{4 m}\right)^{-\frac{3}{2}} \exp \left(-\frac{k_{0}^{2}|\vec{x}-\vec{y}|^{2}}{8+\frac{2 i k_{0}^{2}\left(t-t^{\prime}\right)}{m}}\right) .
$$

Here we have not kept track of the overall normalization. A final fact we require (which is true in the random phase model) is that the following correlators vanish

$$
\left\langle\Psi(\vec{x}, t) \Psi\left(\vec{y}, t^{\prime}\right)\right\rangle=\left\langle\Psi^{*}(\vec{x}, t) \Psi^{*}\left(\vec{y}, t^{\prime}\right)\right\rangle=0
$$

By combining these formulas, we can now compute the statistics of operators built out of the field $\phi$. For example, we have

$$
\left\langle\phi(\vec{x}, t) \phi\left(\vec{y}, t^{\prime}\right)\right\rangle=\frac{1}{2 m}\left\langle\Psi^{*}(\vec{x}, t) \Psi\left(\vec{y}, t^{\prime}\right)\right\rangle e^{i m\left(t-t^{\prime}\right)}+\frac{1}{2 m}\left\langle\Psi(\vec{x}, t) \Psi^{*}\left(\vec{y}, t^{\prime}\right)\right\rangle e^{-i m\left(t-t^{\prime}\right)} .
$$

One can plug (E.4) into this formula to get an explicit expression. Note that when we set $\vec{x}=\vec{y}$ and $t=t^{\prime}$, this becomes the density one-point function:

$$
m^{2}\left\langle\phi^{2}\right\rangle=\bar{\rho} \propto m k_{0}^{3}
$$

The correlator (E.6) is challenging to measure because of the overall oscillatory factors, which vary on a timescale of order $m^{-1}$. In order to overcome this difficulty, we can consider the two-point function of the composite operator $\phi^{2}$, subtracting off the average of $\phi^{2}$ as

$$
\left\langle\phi(\vec{x}, t)^{2} \phi\left(\vec{y}, t^{\prime}\right)^{2}\right\rangle-\left\langle\phi^{2}\right\rangle^{2}=\frac{1}{m^{2}}\left|\left\langle\Psi^{*}(\vec{x}, t) \Psi\left(\vec{y}, t^{\prime}\right)\right\rangle\right|^{2}+\frac{1}{2 m^{2}}\left(\left\langle\Psi^{*}(\vec{x}, t) \Psi\left(\vec{y}, t^{\prime}\right)\right\rangle e^{2 i m\left(t-t^{\prime}\right)}+\text { c.c. }\right),
$$


where c.c. means the complex conjugate of the last term. Plugging in (E.4) we get

$$
\begin{aligned}
\frac{\left\langle\phi(\vec{x}, t)^{2} \phi\left(\vec{y}, t^{\prime}\right)^{2}\right\rangle}{\left\langle\phi^{2}\right\rangle^{2}}-1= & \left(1+\frac{k_{0}^{4}\left(t-t^{\prime}\right)^{2}}{16 m^{2}}\right)^{-3 / 2} \exp \left(-\frac{4 k_{0}^{2} m^{2}|\vec{x}-\vec{y}|^{2}}{16 m^{2}+k_{0}^{4}\left(t-t^{\prime}\right)^{2}}\right) \\
& +\left[\frac{32 m^{3} e^{-2 i m\left(t-t^{\prime}\right)}}{\left[4 m-i k_{0}\left(t-t^{\prime}\right)\right]^{3}} \exp \left(-\frac{i m k_{0}^{2}|\vec{x}-\vec{y}|^{2}}{4 i m+k_{0}^{2}\left(t-t^{\prime}\right)}\right)+\text { c.c. }\right] .
\end{aligned}
$$

The second line of this correlator oscillates with frequency $m^{-1}$, but if we average over times longer than this (but still small compared to the de Broglie time) we can isolate the first line, which is responsible for the behaviors discussed in Section 6.2.

\section{References}

[1] S. Tremaine and J. E. Gunn, "Dynamical Role of Light Neutral Leptons in Cosmology," Phys. Rev. Lett. 42 (1979) 407-410.

[2] A. H. Guth, M. P. Hertzberg, and C. Prescod-Weinstein, "Do Dark Matter Axions Form a Condensate with Long-Range Correlation?," Phys. Rev. D92 (2015) no. 10, 103513, arXiv:1412.5930 [astro-ph.CO].

[3] C.-P. Ma and E. Bertschinger, "Cosmological perturbation theory in the synchronous and conformal Newtonian gauges," Astrophys. J. 455 (1995) 7-25, arXiv:astro-ph/9506072 [astro-ph].

[4] D. Baumann, A. Nicolis, L. Senatore, and M. Zaldarriaga, "Cosmological Non-Linearities as an Effective Fluid," JCAP 1207 (2012) 051, arXiv:1004.2488 [astro-ph.CO].

[5] M. R. Baldeschi, R. Ruffini, and G. B. Gelmini, "On Massive Fermions and Bosons in Galactic Halos," Phys. Lett. 122B (1983) 221-224.

[6] W. H. Press, B. S. Ryden, and D. N. Spergel, "Single Mechanism for Generating Large Scale Structure and Providing Dark Missing Matter," Phys. Rev. Lett. 64 (1990) 1084.

[7] S.-J. Sin, "Late time cosmological phase transition and galactic halo as Bose liquid," Phys. Rev. D50 (1994) 3650-3654, arXiv:hep-ph/9205208 [hep-ph].

[8] P. J. E. Peebles, "Fluid dark matter," Astrophys. J. 534 (2000) L127, arXiv:astro-ph/0002495 [astro-ph].

[9] J. Goodman, "Repulsive dark matter," New Astron. 5 (2000) 103, arXiv:astro-ph/0003018 [astro-ph].

[10] J. Lesgourgues, A. Arbey, and P. Salati, "A light scalar field at the origin of galaxy rotation curves," New Astron. Rev. 46 (2002) 791-799. 
[11] P.-H. Chavanis, "Mass-radius relation of Newtonian self-gravitating Bose-Einstein condensates with short-range interactions: I. Analytical results," Phys. Rev. D84 (2011) 043531, arXiv:1103. 2050 [astro-ph.CO].

[12] A. Suarez and T. Matos, "Structure Formation with Scalar Field Dark Matter: The Fluid Approach," Mon. Not. Roy. Astron. Soc. 416 (2011) 87, arXiv:1101.4039 [gr-qc].

[13] R. D. Peccei and H. R. Quinn, "CP Conservation in the Presence of Instantons," Phys. Rev. Lett. 38 (1977) 1440-1443.

[14] J. E. Kim, "Weak Interaction Singlet and Strong CP Invariance," Phys. Rev. Lett. 43 (1979) 103.

[15] S. Weinberg, “A New Light Boson?," Phys. Rev. Lett. 40 (1978) 223-226.

[16] F. Wilczek, "Problem of Strong $P$ and $T$ Invariance in the Presence of Instantons," Phys. Rev. Lett. 40 (1978) 279-282.

[17] M. A. Shifman, A. I. Vainshtein, and V. I. Zakharov, "Can Confinement Ensure Natural CP Invariance of Strong Interactions?," Nucl. Phys. B166 (1980) 493-506.

[18] A. R. Zhitnitsky, "On Possible Suppression of the Axion Hadron Interactions. (In Russian)," Sov. J. Nucl. Phys. 31 (1980) 260. [Yad. Fiz.31,497(1980)].

[19] M. Dine, W. Fischler, and M. Srednicki, "A Simple Solution to the Strong CP Problem with a Harmless Axion," Phys. Lett. B 104 (1981) 199-202.

[20] J. Preskill, M. B. Wise, and F. Wilczek, "Cosmology of the Invisible Axion," Phys. Lett. 120B (1983) 127-132.

[21] L. Abbott and P. Sikivie, "A Cosmological Bound on the Invisible Axion," Phys. Lett. B 120 (1983) 133-136.

[22] M. Dine and W. Fischler, "The Not So Harmless Axion," Phys. Lett. B 120 (1983) 137-141.

[23] P. W. Graham, I. G. Irastorza, S. K. Lamoreaux, A. Lindner, and K. A. van Bibber, "Experimental Searches for the Axion and Axion-Like Particles," Ann. Rev. Nucl. Part. Sci. 65 (2015) 485-514, arXiv:1602.00039 [hep-ex].

[24] D. J. E. Marsh, “Axion Cosmology,” Phys. Rept. 643 (2016) 1-79, arXiv:1510.07633 [astro-ph.CO].

[25] P. Sikivie, "Invisible Axion Search Methods," arXiv:2003.02206 [hep-ph].

[26] P. Svrcek and E. Witten, "Axions In String Theory," JHEP 06 (2006) 051, arXiv:hep-th/0605206 [hep-th].

[27] A. Arvanitaki, S. Dimopoulos, S. Dubovsky, N. Kaloper, and J. March-Russell, "String Axiverse," Phys. Rev. D81 (2010) 123530, arXiv:0905.4720 [hep-th]. 
[28] J. Halverson, C. Long, and P. Nath, "Ultralight axion in supersymmetry and strings and cosmology at small scales," Phys. Rev. D96 (2017) no. 5, 056025, arXiv:1703.07779 [hep-ph].

[29] T. C. Bachlechner, K. Eckerle, O. Janssen, and M. Kleban, "Axion Landscape Cosmology," JCAP 1909 (2019) no. 09, 062, arXiv:1810.02822 [hep-th].

[30] W. Hu, R. Barkana, and A. Gruzinov, "Cold and fuzzy dark matter," Phys. Rev. Lett. 85 (2000) 1158-1161, arXiv:astro-ph/0003365 [astro-ph].

[31] L. Amendola and R. Barbieri, "Dark matter from an ultra-light pseudo-Goldstone-boson," Phys. Lett. B642 (2006) 192-196, arXiv:hep-ph/0509257.

[32] H.-Y. Schive, T. Chiueh, and T. Broadhurst, "Cosmic Structure as the Quantum Interference of a Coherent Dark Wave," Nature Phys. 10 (2014) 496-499, arXiv:1406.6586 [astro-ph.GA].

[33] J. Veltmaat and J. C. Niemeyer, "Cosmological particle-in-cell simulations with ultralight axion dark matter," Phys. Rev. D94 (2016) no. 12, 123523, arXiv:1608.00802 [astro-ph.C0].

[34] B. Schwabe, J. C. Niemeyer, and J. F. Engels, "Simulations of solitonic core mergers in ultralight axion dark matter cosmologies," Phys. Rev. D94 (2016) no. 4, 043513, arXiv:1606.05151 [astro-ph.CO].

[35] L. Hui, J. P. Ostriker, S. Tremaine, and E. Witten, "Ultralight scalars as cosmological dark matter," Phys. Rev. D95 (2017) no. 4, 043541, arXiv:1610.08297 [astro-ph.Co].

[36] P. Mocz, M. Vogelsberger, V. H. Robles, J. Zavala, M. Boylan-Kolchin, A. Fialkov, and L. Hernquist, "Galaxy formation with BECDM - I. Turbulence and relaxation of idealized haloes," Mon. Not. Roy. Astron. Soc. 471 (2017) no. 4, 4559-4570, arXiv:1705.05845 [astro-ph.CO].

[37] M. Nori and M. Baldi, "AX-GADGET: a new code for cosmological simulations of Fuzzy Dark Matter and Axion models," Mon. Not. Roy. Astron. Soc. 478 (2018) no. 3, 3935-3951, arXiv:1801.08144 [astro-ph.CO].

[38] P. Sikivie and Q. Yang, "Bose-Einstein Condensation of Dark Matter Axions," Phys. Rev. Lett. 103 (2009) 111301, arXiv:0901.1106 [hep-ph].

[39] L. Berezhiani and J. Khoury, "Theory of dark matter superfluidity," Phys. Rev. D92 (2015) 103510, arXiv:1507.01019 [astro-ph.CO].

[40] J. Fan, "Ultralight Repulsive Dark Matter and BEC," Phys. Dark Univ. 14 (2016) 84-94, arXiv:1603.06580 [hep-ph].

[41] S. Alexander and S. Cormack, "Gravitationally bound BCS state as dark matter," JCAP 1704 (2017) no. 04, 005, arXiv:1607.08621 [astro-ph.CO].

[42] S. Alexander, E. McDonough, and D. N. Spergel, "Chiral Gravitational Waves and Baryon Superfluid Dark Matter," JCAP 05 (2018) 003, arXiv:1801.07255 [hep-th]. 
[43] T. Jacobson, "Primordial black hole evolution in tensor scalar cosmology," Phys. Rev. Lett. 83 (1999) 2699-2702, arXiv:astro-ph/9905303 [astro-ph].

[44] A. Arvanitaki and S. Dubovsky, "Exploring the String Axiverse with Precision Black Hole Physics," Phys. Rev. D83 (2011) 044026, arXiv:1004.3558 [hep-th].

[45] C. F. B. Macedo, P. Pani, V. Cardoso, and L. C. B. Crispino, "Into the lair: gravitational-wave signatures of dark matter," Astrophys. J. 774 (2013) 48, arXiv:1302.2646 [gr-qc].

[46] L. Hui, D. Kabat, X. Li, L. Santoni, and S. S. C. Wong, "Black Hole Hair from Scalar Dark Matter," JCAP 1906 (2019) no. 06, 038, arXiv:1904.12803 [gr-qc].

[47] K. Clough, P. G. Ferreira, and M. Lagos, "Growth of massive scalar hair around a Schwarzschild black hole," Phys. Rev. D100 (2019) no. 6, 063014, arXiv:1904.12783 [gr-qc].

[48] D. Baumann, H. S. Chia, J. Stout, and L. ter Haar, "The Spectra of Gravitational Atoms," JCAP 1912 (2019) no. 12, 006, arXiv:1908.10370 [gr-qc].

[49] K. Mukaida, M. Takimoto, and M. Yamada, "On Longevity of I-ball/Oscillon," JHEP 03 (2017) 122, arXiv:1612.07750 [hep-ph].

[50] M. H. Namjoo, A. H. Guth, and D. I. Kaiser, "Relativistic Corrections to Nonrelativistic Effective Field Theories," Phys. Rev. D98 (2018) no. 1, 016011, arXiv:1712.00445 [hep-ph].

[51] J. Eby, K. Mukaida, M. Takimoto, L. Wijewardhana, and M. Yamada, "Classical nonrelativistic effective field theory and the role of gravitational interactions," Phys. Rev. D 99 (2019) no. 12, 123503, arXiv:1807.09795 [hep-ph].

[52] D. J. E. Marsh and P. G. Ferreira, "Ultra-Light Scalar Fields and the Growth of Structure in the Universe," Phys. Rev. D82 (2010) 103528, arXiv:1009.3501 [hep-ph].

[53] R. Hlozek, D. Grin, D. J. E. Marsh, and P. G. Ferreira, "A search for ultralight axions using precision cosmological data," Phys. Rev. D91 (2015) no. 10, 103512, arXiv:1410.2896 [astro-ph.CO].

[54] X. Li, L. Hui, and G. L. Bryan, "Numerical and Perturbative Computations of the Fuzzy Dark Matter Model," Phys. Rev. D99 (2019) no. 6, 063509, arXiv:1810.01915 [astro-ph.C0].

[55] E. Madelung, "Quantentheorie in hydrodynamischer form," Zeitschrift für Physik 40 (Mar, 1927) 322-326.

[56] R. P. Feynman, R. B. Leighton, and M. Sands, The Feynman Lectures on Physics. 1963. http://www. feynmanlectures.info/.

[57] W. Thomson, "Vi. - on vortex motion," Transactions of the Royal Society of Edinburgh 25 (1868) no. 1, 217-260. 
[58] B. Damski and K. Sacha, "Changes of the topological charge of vortices," Journal of Physics A: Mathematical and General 36 (feb, 2003) 2339-2345.

[59] H. M. Nilsen, G. Baym, and C. J. Pethick, "Velocity of vortices in inhomogeneous bose-einstein condensates," Proceedings of the National Academy of Sciences 103 (2006) no. 21, 7978-7981.

[60] L. M. Widrow and N. Kaiser, "Using the Schrodinger equation to simulate collisionless matter," Astrophys. J. 416 (1993) L71-L74.

[61] P. Mocz, L. Lancaster, A. Fialkov, F. Becerra, and P.-H. Chavanis, "Schrödinger-Poisson-Vlasov-Poisson correspondence," Phys. Rev. D97 (2018) no. 8, 083519 , arXiv:1801.03507 [astro-ph.CO].

[62] C. Uhlemann, C. Rampf, M. Gosenca, and O. Hahn, "A semiclassical path to cosmic large-scale structure," arXiv:1812.05633 [astro-ph.C0].

[63] H. B. Nielsen and P. Olesen, "Vortex Line Models for Dual Strings," Nucl. Phys. B61 (1973) 45-61.

[64] M. Luscher, "Symmetry Breaking Aspects of the Roughening Transition in Gauge Theories," Nucl. Phys. B180 (1981) 317-329.

[65] L. Onsager, "Statistical hydrodynamics," Il Nuovo Cimento 6 (Mar., 1949) 279-287.

[66] A. L. Fetter, "Rotating trapped Bose-Einstein condensates," Laser Physics 18 (Jan, 2008) 1-11, arXiv:0801.2952 [cond-mat.stat-mech].

[67] J. Polchinski and A. Strominger, "Effective string theory," Phys. Rev. Lett. 67 (1991) 1681-1684.

[68] F. Lund, "Defect dynamics for the nonlinear schrödinger equation derived from a variational principle," Physics Letters A 159 (1991) no. 4, 245 - 251.

[69] S. Dubovsky, R. Flauger, and V. Gorbenko, "Effective String Theory Revisited," JHEP 09 (2012) 044, arXiv:1203.1054 [hep-th].

[70] O. Aharony and Z. Komargodski, "The Effective Theory of Long Strings," JHEP 05 (2013) 118, arXiv:1302.6257 [hep-th].

[71] B. Horn, A. Nicolis, and R. Penco, "Effective string theory for vortex lines in fluids and superfluids," JHEP 10 (2015) 153, arXiv:1507.05635 [hep-th].

[72] M. P. Silverman and R. L. Mallett, "Dark matter as a cosmic Bose-Einstein condensate and possible superfluid," Gen. Rel. Grav. 34 (2002) 633-649.

[73] M. N. Brook and P. Coles, "Gravitational Stability of Vortices in Bose-Einstein Condensate Dark Matter," arXiv:0902.0605 [astro-ph.C0].

[74] B. Kain and H. Y. Ling, "Vortices in Bose-Einstein Condensate Dark Matter," Phys. Rev. D82 (2010) 064042, arXiv:1004.4692 [hep-ph]. 
[75] T. Rindler-Daller and P. R. Shapiro, "Angular Momentum and Vortex Formation in Bose-Einstein-Condensed Cold Dark Matter Haloes," Mon. Not. Roy. Astron. Soc. 422 (2012) 135-161, arXiv:1106.1256 [astro-ph.C0].

[76] N. T. Zinner, "Vortex Structures in a Rotating BEC Dark Matter Component," Phys. Res. Int. 2011 (2011) 734543, arXiv:1108.4290 [astro-ph.C0].

[77] N. Banik and P. Sikivie, "Axions and the Galactic Angular Momentum Distribution," Phys. Rev. D88 (2013) 123517, arXiv:1307.3547 [astro-ph.GA].

[78] T. Chiueh, T.-P. Woo, H.-Y. Jian, and H.-Y. Schive, "Vortex turbulence in linear Schrödinger wave mechanics," Journal of Physics B Atomic Molecular Physics 44 (June, 2011) 115101, arXiv:0910.2791 [quant-ph].

[79] J. H. H. Chan, H.-Y. Schive, S.-K. Wong, and T. Chiueh, "Multiple Images and Flux Ratio Anomaly of Fuzzy Gravitational Lenses,"arXiv e-prints (Feb., 2020) arXiv:2002.10473, arXiv:2002.10473 [astro-ph.GA].

[80] P. A. M. Dirac, "Quantized Singularities in the Electromagnetic Field," Proc. Roy. Soc. Lond. A133 (1931) 60-72.

[81] P. Duren, W. Hengartner, and R. S. Laugesen, "The argument principle for harmonic functions," The American Mathematical Monthly 103 (1996) no. 5, 411-415. http://www. jstor.org/stable/2974933.

[82] I. Bialynicki-Birula, Z. Bialynicka-Birula, and C. Śliwa, "Motion of vortex lines in quantum mechanics," Phys. Rev. A 61 (Mar., 2000) 032110, quant-ph/9911007.

[83] U. Niederer, "The maximal kinematical invariance group of the free Schrodinger equation.," Helv. Phys. Acta 45 (1972) 802-810.

[84] D. T. Son, "Toward an AdS/cold atoms correspondence: A Geometric realization of the Schrodinger symmetry," Phys. Rev. D78 (2008) 046003, arXiv:0804.3972 [hep-th].

[85] A. Derevianko, "Detecting dark-matter waves with a network of precision-measurement tools," Phys. Rev. A97 (2018) no. 4, 042506, arXiv:1605.09717 [physics.atom-ph].

[86] J. W. Foster, N. L. Rodd, and B. R. Safdi, "Revealing the Dark Matter Halo with Axion Direct Detection," Phys. Rev. D97 (2018) no. 12, 123006, arXiv:1711.10489 [astro-ph.Co].

[87] G. P. Centers et al., "Stochastic fluctuations of bosonic dark matter," arXiv:1905.13650 [astro-ph. CO].

[88] J. M. Bardeen, J. R. Bond, N. Kaiser, and A. S. Szalay, "The Statistics of Peaks of Gaussian Random Fields," Astrophys. J. 304 (1986) 15-61. 
[89] H.-Y. Schive, M.-H. Liao, T.-P. Woo, S.-K. Wong, T. Chiueh, T. Broadhurst, and W. Y. P. Hwang, "Understanding the Core-Halo Relation of Quantum Wave Dark Matter from 3D Simulations," Phys. Rev. Lett. 113 (2014) no. 26, 261302, arXiv:1407.7762 [astro-ph.GA].

[90] P. Mocz and S. Succi, "Numerical solution of the nonlinear Schrödinger equation using smoothed-particle hydrodynamics," Phys. Rev. E91 (2015) no. 5, 053304, arXiv:1503.03869 [physics.comp-ph].

[91] J. Veltmaat, J. C. Niemeyer, and B. Schwabe, "Formation and structure of ultralight bosonic dark matter halos," Phys. Rev. D98 (2018) no. 4, 043509, arXiv:1804.09647 [astro-ph.C0].

[92] F. Edwards, E. Kendall, S. Hotchkiss, and R. Easther, "PyUltraLight: A Pseudo-Spectral Solver for Ultralight Dark Matter Dynamics," JCAP 1810 (2018) no. 10, 027, arXiv:1807.04037 [astro-ph.CO].

[93] M. Ester, H.-P. Kriegel, J. Sander, and X. Xu, "A density-based algorithm for discovering clusters in large spatial databases with noise," pp. 226-231. AAAI Press, 1996.

[94] M. Bartelmann, "Gravitational Lensing," Class. Quant. Grav. 27 (2010) 233001, arXiv:1010. 3829 [astro-ph.CO].

[95] S.-d. Mao and P. Schneider, "Evidence for substructure in lens galaxies?," Mon. Not. Roy. Astron. Soc. 295 (1998) 587-594, arXiv:astro-ph/9707187 [astro-ph].

[96] M. Chiba, "Probing dark matter substructure in lens galaxies," Astrophys. J. 565 (2002) 17, arXiv:astro-ph/0109499 [astro-ph].

[97] R. B. Metcalf and P. Madau, "Compound gravitational lensing as a probe of dark matter substructure within galaxy halos," Astrophys. J. 563 (2001) 9, arXiv:astro-ph/0108224 [astro-ph].

[98] N. Dalal and C. S. Kochanek, "Direct detection of CDM substructure," Astrophys. J. 572 (2002) 25-33, arXiv:astro-ph/0111456 [astro-ph].

[99] C. S. Kochanek and N. Dalal, "Tests for substructure in gravitational lenses," Astrophys. J. 610 (2004) 69-79, arXiv:astro-ph/0302036 [astro-ph].

[100] S. Mao, Y.-P. Jing, J. P. Ostriker, and J. Weller, "Anomalous flux ratios in gravitational lenses: For or against CDM?," Astrophys. J. 604 (2004) L5-L8, arXiv:astro-ph/0402149 [astro-ph].

[101] A. Amara, R. B. Metcalf, T. J. Cox, and O. J. P., "Simulations of strong gravitational lensing with substructure," Mon. Not. Roy. Astron. Soc. 367 (2006) 1367-1378, arXiv:astro-ph/0411587 [astro-ph].

[102] Y. D. Hezaveh et al., "Detection of lensing substructure using ALMA observations of the dusty galaxy SDP.81," Astrophys. J. 823 (2016) no. 1, 37, arXiv:1601.01388 [astro-ph.C0]. 
[103] S. Alexander, J. J. Bramburger, and E. McDonough, "Dark Disk Substructure and Superfluid Dark Matter," Phys. Lett. B 797 (2019) 134871, arXiv:1901.03694 [astro-ph.CO].

[104] S. Alexander, S. Gleyzer, E. McDonough, M. W. Toomey, and E. Usai, "Deep Learning the Morphology of Dark Matter Substructure," Astrophys. J. 893 (2020) 15, arXiv: 1909.07346 [astro-ph.CO].

[105] A. Maleki, S. Baghram, and S. Rahvar, "Investigation of two colliding solitonic cores in Fuzzy Dark Matter models," Phys. Rev. D 101 (2020) no. 2, 023508, arXiv:1911.00486 [astro-ph.C0].

[106] P. Schneider, J. Ehlers, and E. E. Falco, Gravitational Lenses. 1992.

[107] L. Dai, A. A. Kaurov, K. Sharon, M. K. Florian, J. Miralda-Escudé, T. Venumadhav, B. Frye, J. R. Rigby, and M. Bayliss, "Asymmetric Surface Brightness Structure of Lensed Arc in SDSS J1226+2152: A Case for Dark Matter Substructure," arXiv:2001.00261 [astro-ph.GA] .

[108] Y. Hezaveh, N. Dalal, G. Holder, T. Kisner, M. Kuhlen, and L. Perreault Levasseur, "Measuring the power spectrum of dark matter substructure using strong gravitational lensing," JCAP 1611 (2016) no. 11, 048, arXiv:1403.2720 [astro-ph.c0].

[109] A. Diaz Rivero, F.-Y. Cyr-Racine, and C. Dvorkin, "Power spectrum of dark matter substructure in strong gravitational lenses," Phys. Rev. D97 (2018) no. 2, 023001, arXiv:1707.04590 [astro-ph.CO].

[110] K. Van Tilburg, A.-M. Taki, and N. Weiner, "Halometry from Astrometry," JCAP 1807 (2018) no. 07, 041, arXiv:1804.01991 [astro-ph.C0].

[111] C. Mondino, A.-M. Taki, K. Van Tilburg, and N. Weiner, "First Results on Dark Matter Substructure from Astrometric Weak Lensing," arXiv:2002.01938 [astro-ph.c0].

[112] S. Mishra-Sharma, K. Van Tilburg, and N. Weiner, "The Power of Halometry," arXiv:2003.02264 [astro-ph.CO].

[113] I. G. Irastorza and J. Redondo, "New experimental approaches in the search for axion-like particles," Prog. Part. Nucl. Phys. 102 (2018) 89-159, arXiv:1801.08127 [hep-ph].

[114] P. W. Graham and S. Rajendran, "New Observables for Direct Detection of Axion Dark Matter," Phys. Rev. D 88 (2013) 035023, arXiv:1306.6088 [hep-ph].

[115] ADMX , N. Du et al., "A Search for Invisible Axion Dark Matter with the Axion Dark Matter Experiment," Phys. Rev. Lett. 120 (2018) no. 15, 151301, arXiv:1804.05750 [hep-ex].

[116] P. Sikivie, "Experimental tests of the invisible axion," Phys. Rev. Lett. 51 (1983) 1415-1417. [Erratum: Phys. Rev. Lett. 52, 695 (1984)].

[117] Y. Kahn, B. R. Safdi, and J. Thaler, "Broadband and Resonant Approaches to Axion Dark Matter Detection," Phys. Rev. Lett. 117 (2016) no. 14, 141801, arXiv:1602.01086 [hep-ph]. 
[118] J. L. Ouellet et al., "First Results from ABRACADABRA-10 cm: A Search for Sub- $\mu$ eV Axion Dark Matter," Phys. Rev. Lett. 122 (2019) no. 12, 121802, arXiv:1810.12257 [hep-ex].

[119] H. Liu, B. D. Elwood, M. Evans, and J. Thaler, "Searching for Axion Dark Matter with Birefringent Cavities," Phys. Rev. D100 (2019) no. 2, 023548, arXiv:1809.01656 [hep-ph].

[120] E. Savalle, B. M. Roberts, F. Frank, P.-E. Pottie, B. T. McAllister, C. Dailey, A. Derevianko, and P. Wolf, "Novel approaches to dark-matter detection using space-time separated clocks," arXiv:1902.07192 [gr-qc].

[121] D. Martynov and H. Miao, "Quantum-enhanced interferometry for axion searches," Phys. Rev. D 101 (2020) no. 9, 095034, arXiv:1911.00429 [physics.ins-det].

[122] D. Budker, P. W. Graham, M. Ledbetter, S. Rajendran, and A. Sushkov, "Proposal for a Cosmic Axion Spin Precession Experiment (CASPEr)," Phys. Rev. X4 (2014) no. 2, 021030, arXiv:1306.6089 [hep-ph].

[123] D. F. Jackson Kimball et al., "Overview of the Cosmic Axion Spin Precession Experiment (CASPEr)," arXiv:1711.08999 [physics.ins-det].

[124] W. A. Terrano, E. G. Adelberger, C. A. Hagedorn, and B. R. Heckel, "Constraints on axionlike dark matter with masses down to $10^{-23} \mathrm{eV} / \mathrm{c}^{2}$," Phys. Rev. Lett. 122 (2019) no. 23, 231301, arXiv:1902.04246 [astro-ph.CO].

[125] M. P. Hertzberg and E. D. Schiappacasse, "Scalar dark matter clumps with angular momentum," JCAP 1808 (2018) no. 08, 028, arXiv:1804.07255 [hep-ph].

[126] N. C. Amorisco and A. Loeb, "First constraints on Fuzzy Dark Matter from the dynamics of stellar streams in the Milky Way," arXiv:1808.00464 [astro-ph.GA].

[127] B. V. Church, J. P. Ostriker, and P. Mocz, "Heating of Milky Way disc Stars by Dark Matter Fluctuations in Cold Dark Matter and Fuzzy Dark Matter Paradigms," Mon. Not. Roy. Astron. Soc. 485 (2019) no. 2, 2861-2876, arXiv:1809.04744 [astro-ph.GA].

[128] B. Bar-Or, J.-B. Fouvry, and S. Tremaine, "Relaxation in a Fuzzy Dark Matter Halo," Astrophys. J. 871 (2019) no. 1, 28, arXiv:1809.07673 [astro-ph.GA].

[129] D. J. E. Marsh and J. C. Niemeyer, "Strong Constraints on Fuzzy Dark Matter from Ultrafaint Dwarf Galaxy Eridanus II," Phys. Rev. Lett. 123 (2019) no. 5, 051103, arXiv:1810.08543 [astro-ph.CO]. 